\title{
Experimental and Numerical Study of a Hydrogen Peroxide / Hydroxyl Terminated Polybutadiene Hybrid Rocket
}

by

Erin Farbar, B. Eng.

A thesis submitted to the Faculty of Graduate Studies and Research in partial fulfillment of the requirements for the degree of

Master of Applied Science

Ottawa-Carleton Institute for Mechanical and Aerospace Engineering

Department of Mechanical and Aerospace Engineering

\section{Carleton University}

Ottawa, Ontario, Canada

November 25, 2005 


$\begin{array}{ll}\begin{array}{l}\text { Library and } \\ \text { Archives Canada }\end{array} & \begin{array}{l}\text { Bibliothèque et } \\ \text { Archives Canada }\end{array} \\ \begin{array}{l}\text { Published Heritage } \\ \text { Branch }\end{array} & \begin{array}{l}\text { Direction du } \\ \text { Patrimoine de l'édition }\end{array} \\ \begin{array}{l}\text { 395 Wellington Street } \\ \text { Ottawa ON K1A ON4 }\end{array} & \begin{array}{l}\text { 395, rue Wellington } \\ \text { Ottawa ON K1A ON4 } \\ \text { Canada }\end{array}\end{array}$

Your file Votre référence

ISBN: 0-494-13439-9

Our file Notre référence

ISBN: 0-494-13439-9

NOTICE:

The author has granted a nonexclusive license allowing Library and Archives Canada to reproduce, publish, archive, preserve, conserve, communicate to the public by telecommunication or on the Internet, loan, distribute and sell theses worldwide, for commercial or noncommercial purposes, in microform, paper, electronic and/or any other formats.

The author retains copyright ownership and moral rights in this thesis. Neither the thesis nor substantial extracts from it may be printed or otherwise reproduced without the author's permission.
AVIS:

L'auteur a accordé une licence non exclusive permettant à la Bibliothèque et Archives Canada de reproduire, publier, archiver, sauvegarder, conserver, transmettre au public par télécommunication ou par l'Internet, prêter, distribuer et vendre des thèses partout dans le monde, à des fins commerciales ou autres, sur support microforme, papier, électronique et/ou autres formats.

L'auteur conserve la propriété du droit d'auteur et des droits moraux qui protège cette thèse. $\mathrm{Ni}$ la thèse ni des extraits substantiels de celle-ci ne doivent être imprimés ou autrement reproduits sans son autorisation.
In compliance with the Canadian

Privacy Act some supporting forms may have been removed from this thesis.

While these forms may be included in the document page count, their removal does not represent any loss of content from the thesis.
Conformément à la loi canadienne sur la protection de la vie privée, quelques formulaires secondaires ont été enlevés de cette thèse.

Bien que ces formulaires aient inclus dans la pagination, il n'y aura aucun contenu manquant.

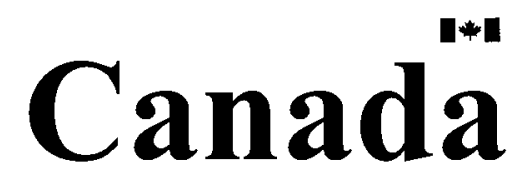




\section{Acknowledgments}

First and foremost I thank Anthony Cesaroni, Fred Christie and Richard Worsfold for affording me the opportunity to work at CTI during the summer of 2004. I am indebted to Dr. Jeroen Louwers for working with me on the HyperG experiments and for his unending patience. Also, I must thank Cleon, Fiona, Jimmy and Dave at CTI for offering their help whenever it was needed.

Of course, I thank Dr. Tarik Kaya for his support and flexibility in completing this project. Additionally, I thank my aunt and grandmother, without whom I would not have attended Carleton in the first place.

Lastly, thank you to Tyson Ferguson, for his troubleshooting expertise with ANSYS CFX, and for always being a listening ear.

ERIN FARBAR

Carleton University

November 25, 2005

iii 


\section{Abstract}

Results from fifteen static test firings of lab scale hybrid rocket motors utilizing $90 \%$ concentrated hydrogen peroxide as an oxidizer with hydroxyl terminated polybutadiene fuel are presented. Thirteen of those tests utilized aluminum or aluminum/magnesium alloy additive in the fuel. The performance characteristics of the propellant combinations were determined. The experimental results indicated combustion efficiencies of $72 \%-90 \%$, and regression rates on the order of $1 \mathrm{~mm} / \mathrm{s}$ for the metallized fuel combinations. A correlation of the regression rate data for the metallized propellants deviated from that which was expected for turbulent convective heat transfer dominated behaviour, indicating a possible sensitivity of the

metallized propellant combinations to chamber pressure. A numerical model of the hydrogen peroxide/non-metallized hydroxyl terminated polybutadiene motor was built in a commercial computational fluid dynamics code, CFX 5.7.1. The model was combined with a FORTRAN code that was developed at Carleton University, to predict the regression rate of the propellant combination at the initial operating conditions for two of the experimental tests. The predicted spatially averaged regression rates agreed with the measured spatially and temporally averaged regression rates to within $6 \%$ and $10 \%$ respectively.

iv 


\section{Contents}

$\begin{array}{ll}\text { Acknowledgments } & \text { iii }\end{array}$

Abstract iv

$\begin{array}{ll}\text { List of Tables } & \text { ix }\end{array}$

List of Figures $\quad$ xi

Nomenclature $\quad x v i$

$\begin{array}{lll}\text { Chapter } 1 \text { Introduction } & 1\end{array}$

1.1 Background . . . . . . . . . . . . . . . . . . . 1

1.2 Theory............................. 3

1.2.1 Rocket theory . . . . . . . . . . . . . . . . 4

1.2 .2 Hybrid rocket theory . . . . . . . . . . . 8

1.3 Previous experimental studies of hybrid rocket motors . . . . . . 14

1.4 Previous numerical studies of hybrid rocket motors . . . . . . . . . 26 
1.5 Summary . . . . . . . . . . . . . . . . . . . . 31

$\begin{array}{lll}\text { Chapter } 2 & \text { Experimental Program } & 33\end{array}$

2.1 Program objectives . . . . . . . . . . . . . 33

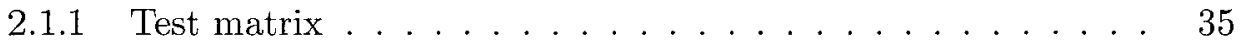

2.2 Theoretical performance calculations . . . . . . . . . 36

2.3 Propellant . . . . . . . . . . . . . . . . . . . 40

2.3.1 Hydrogen peroxide specifications . . . . . . . . . . . . . 40

2.3 .2 Fuel formulations . . . . . . . . . . . . . . . . . . . 41

2.4 Test hardware . . . . . . . . . . . . . . . . . . . 42

2.4 .1 Catalyst pack . . . . . . . . . . . . . . 42

2.4 .2 Rocket motor components . . . . . . . . . . . . . . 43

2.4.3 Thrust bench hardware and instrumentation . . . . . . 48

2.4.4 Venturi calibration . . . . . . . . . . . . 50

2.4 .5 Data acquisition . . . . . . . . . . . . 55

2.5 Test procedure . . . . . . . . . . . . . . 55

2.6 Data reduction . . . . . . . . . . . . . . . . 57

2.6 .1 Time averaged analysis .............. 60

2.6 .2 Error analysis . . . . . . . . . . . . . . . . 64

2.7 Results and discussion . . . . . . . . . . . . . 65

vi 
2.7 .1 Ignition transient . . . . . . . . . . . . 67

2.7 .2 Pressure and thrust anomalies . . . . . . . . . 67

2.7 .3 Qualitative observations . . . . . . . . . . . 70

2.7.4 Combustion instability . . . . . . . . . . . . . 73

2.7 .5 Nozzle erosion . . . . . . . . . . . . . . . . 77

2.7 .6 Regression rate . . . . . . . . . . . . . 78

2.7 .7 Combustion efficiency . . . . . . . . . . . 84

$\begin{array}{lll}\text { Chapter } 3 & \text { Numerical Modeling } & 92\end{array}$

3.1 Objectives . . . . . . . . . . . . . . . . . . 92

3.2 Model overview . . . . . . . . . . . . . . . . . . . . . . . . . . . 94

3.3 Solution of the HRM model using CFX $5.7 .1 \ldots \ldots 6$

3.3 .1 Governing equations . . . . . . . . . . . . . 96

3.3.2 Discretization and solution methodology . . . . . . . . . 99

3.3 .3 Wall functions . . . . . . . . . . . . . . . . . 100

3.3.4 Mesh generation and refinement . . . . . . . . 105

3.3.5 Boundary conditions and other model parameters . . . . 112

3.3 .6 Solver control . . . . . . . . . . . . . . . . . . . 114

3.4 HRM submodels . . . . . . . . . . . . . . . . 115

3.4.1 Material models .................. 115

vii 
3.4 .2 Turbulence model . . . . . . . . . . . . . . . . 117

3.4.3 Combustion model . . . . . . . . . . . . . . . . . . 122

3.4.4 Radiation model . . . . . . . . . . . . . . . . . . . 125

3.4.5 Fuel ablation model . . . . . . . . . . . . . . . . . . 128

3.4 .6 CFX/FORTRAN interface $\ldots \ldots \ldots \ldots . \ldots 130$

3.5 Results. . . . . . . . . . . . . . . . . . . 131

3.5.1 Velocity, density and temperature fields . . . . . . . . . 132

3.5.2 Contributions to wall heat flux . . . . . . . . . . 136

3.5.3 Variable values at $0.5 \mathrm{~L} \ldots \ldots \ldots \ldots \ldots$

3.5.4 Fuel regression rate, mass injection rate and surface temperature146

3.5.5 Comparison of numerical and experimental results . . . . . 149

Chapter 4 Summary and Conclusions

4.1 Experimental Results . . . . . . . . . . . . . . . . . . 153

4.2 Numerical Results . . . . . . . . . . . . . . . . . . . . . 157

4.3 Recommendations . . . . . . . . . . . . . . . . . . 160

4.3 .1 Experimental program . . . . . . . . . . . . . 160

4.3 .2 Numerical model . . . . . . . . . . . . . . . . . . . . . . . . 161

Bibliography

viii 


\section{List of Tables}

2.1 Metallic particle characteristics. . . . . . . . . . . . . . . 41

2.2 Fuel additives and their uses. . . . . . . . . . . . . . . . . 42

2.3 Materials of construction for the HyperG motor and catalyst pack components. . . . . . . . . . . . . . . . . . . 48

2.4 Parameter uncertainty values used in error analysis. . . . . . . . 66

2.5 Experimental errors for Test $14 \ldots \ldots \ldots 66$

2.6 Nozzle throat erosion for various Fuel/90\%HP propellant combinations. 78

2.7 Summary of combustion efficiencies of various Fuel/90\%HP combinations and data from other relevant lab scale hybrid motor experiments. 86

3.1 Mesh statistics used during refinement. . . . . . . . . . . . . . . 112

3.2 Mesh statistics for HRM Model. . . . . . . . . . . . . . . . . . . 112

3.3 Model parameters and boundary conditions. . . . . . . . . . . . . 114

3.4 Solver control parameters. . . . . . . . . . . . . . . . . 115

ix 
3.5 Constants for the SST turbulence model. . . . . . . . . . . . . 121

3.6 Summary of coefficients for the grey gas spectral model. . . . . . . . 127

3.7 Summary of quantities specified at each subdomain source. . . . . 130

3.8 Data for step heights in HTPB14 and HTPB15 models. . . . . . . . 147

3.9 Comparison of experimental and numerical results: regression rate. . 151

3.10 Comparison of experimental and numerical results: maximum flame temperature. . . . . . . . . . . . . . 152

3.11 Comparison of experimental and numerical results: flame location at

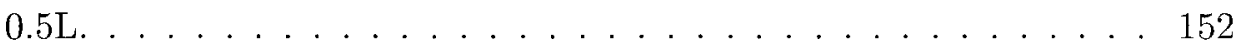




\section{List of Figures}

1.1 Simplified diagram of a hybrid rocket motor[1]. . . . . . . . . 4

1.2 Energy balance at the fuel surface in a hybrid rocket motor[1] . . . 10

2.1 Test matrix for the HyperG program . . . . . . . . . . . 36

2.2 Theoretical performance of HyperG propellants: ISP . . . . . . 38

2.3 Theoretical performance of HyperG propellants: $c^{*} \ldots 39$

2.4 Catalyst pack and injector components. . . . . . . . . . . 44

2.5 Catalyst pack and injector assembly. . . . . . . . . . . 44

2.6 Support for catalyst screens. . . . . . . . . . . . . . 45

2.7 Decomposition of $45 \% \mathrm{HP}$ on catalyst screen. . . . . . . . 45

2.8 Cases, liner, and forward and aft closures for Pro75 hobby rocket line. 46

2.9 Assembly drawing of the HyperG motor. . . . . . . . . . . . 47

2.10 Schematic of the HyperG thrust bench assembly. . . . . . . . . . 51

2.11 Photograph of the HyperG assembly in the test cell. . . . . . . . . 52

$\mathrm{xi}$ 
2.12 Data for Test 1 showing the lag of the $\mathrm{P} 4$ transducer at the start of

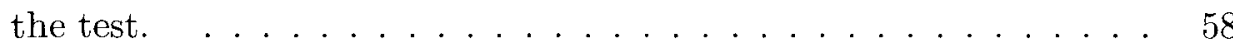

$2.130 .05 \mathrm{~s}$ of data for Test 1 showing the small difference between the P3 and $\mathrm{P} 4$ transducer readings. . . . . . . . . . . . . . . . 59

2.14 Data from all channels, Test $10 . \ldots \ldots$. . . . . . . . . 68

2.15 Experimental results for Tests $1-15 \ldots \ldots \ldots 69$

2.16 Slag agglomeration on a nozzle from Test $1 . \ldots \ldots 71$

2.17 Aluminum oxide powder on a spent fuel grain. . . . . . . . . . 72

2.18 Hyper $G$ nozzles with slag agglomeration. From left to right, top to bottom: new, Test 3 , Test 10, Test 15. . . . . . . . . 73

2.19 Combustion instability in Test 3, elimination of combustion instability in Tests $6,7 \ldots \ldots \ldots \ldots \ldots \ldots \ldots \ldots$

2.20 Regression rate data from all HyperG tests, comparable data from other experiments using lab scale hybrid motors and HP oxidizer. . 81

2.21 Qualitative illustration of the effects of pressure and total mass flux variations on the regression rate in a hybrid motor. . . . . . . 84

2.22 Combustion efficiency as a function of OF for $60 \% \mathrm{Al} / 40 \% \mathrm{HTPB} / 90 \% \mathrm{HP} . \ldots \ldots \ldots \ldots$

2.23 Combustion efficiency as a function of OF for $60 \% \mathrm{AlMg} / 40 \% \mathrm{HTPB} / 90 \%$ HP. . . . . . . . . . . . . . 90 
2.24 Combustion efficiency as a function of OF for HTPB $/ 90 \%$ HP. . . . . . . . . . . . . . . . 91

3.1 Velocity overshoot and lack of log-law velocity profile typical of a turbulent boundary layer with blowing and combustion [48] . . . . . 101

3.2 Illustration of the application of a subdomain source in CFX 5.7.1. . 105

3.3 Effect of suction or blowing on local Stanton number in a turbulent boundary layer over a flat plate $[47] \ldots \ldots \ldots \ldots$

3.4 Change in regression rate along the length of the grain as the mesh was refined. . . . . . . . . . . . . . . . . . 108

3.5 Change in temperature profile at $0.5 \mathrm{~L}$ as the mesh was refined. . . . 109

3.6 Change in regression rate with mesh refinement relative to nominal mesh. ........................ . . 110

3.7 XZ plane showing mesh and coordinate axes for the HTPB14 model. 111

3.8 View of source number ten in the HTPB14 model. Source was $0.001 \mathrm{~mm}$ thick. . . . . . . . . . . . . . . . . . . 129

3.9 HTPB14 - Contours of the axial velocity component $[\mathrm{m} / \mathrm{s}] . \ldots 133$

3.10 HTPB 14 - Temperature contours $[\mathrm{K}] . \ldots \ldots . . \ldots 134$

3.11 HTPB14 - Density contours $\left[\mathrm{kg} / \mathrm{m}^{3}\right] \ldots \ldots \ldots$

3.12 HTPB14 - Variation of wall heat flux in motor axial direction. . . . 137

3.13 HTPB15 - Variation of wall heat flux in motor axial direction. . . . 138 xiii 
3.14 Contribution of convection to the total wall heat flux for HTPB14

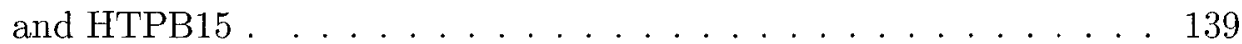

3.15 HTPB14 - Variation of density, temperature and axial velocity component normal to the fuel surface at $0.5 \mathrm{~L} \ldots \ldots \ldots \ldots$

3.16 HTPB14 - Variation of component mass fractions normal to the fuel surface at $0.5 \mathrm{~L} \ldots \ldots \ldots \ldots \ldots \ldots \ldots \ldots \ldots$

3.17 HTPB14 - Variation of dynamic viscosity, thermal conductivity and Prandtl number normal to the fuel surface at $0.5 \mathrm{~L} \ldots \ldots \ldots$. . . . . 142

3.18 HTPB14 - Variation of turbulence kinetic energy, eddy dissipation and eddy viscosity normal to the fuel surface at $0.5 \mathrm{~L} . \ldots \ldots 143$

3.19 Comparison of temperature profiles as a function of absolute wall distance at 0.5L for HTPB14 and HTPB15. . . . . . . . . . . 144

3.20 Comparison of temperature profiles as a function of non-dimensional wall distance at $0.5 \mathrm{~L}$ for HTPB14 and HTPB15. . . . . . . 145

3.21 Vortex at fuel edge due to forward facing step geometry in motor. . 147

3.22 HTPB14 - Variation of regression rate, surface temperature and mass injection rate in the axial direction. Dashed lines indicate experimental error envelope. . . . . . . . . . . . . . . . . . . . . . . 149 
3.23 HTPB15 - Variation of regression rate, surface temperature and mass injection rate in the axial direction. Dashed lines indicate experimental error envelope. . . . . . . . . . . . . . . . 150 


\section{Nomenclature}

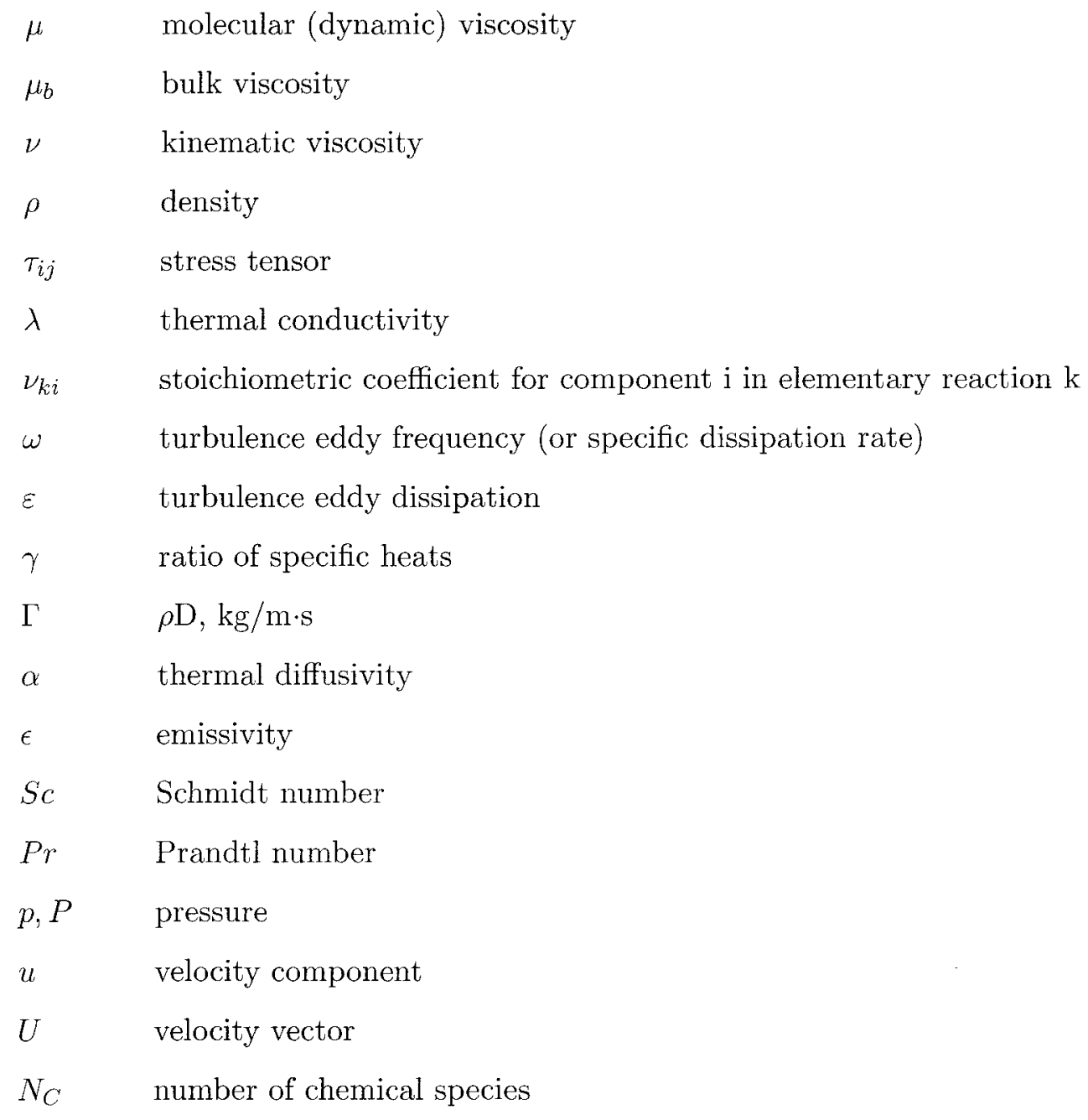

xvi 


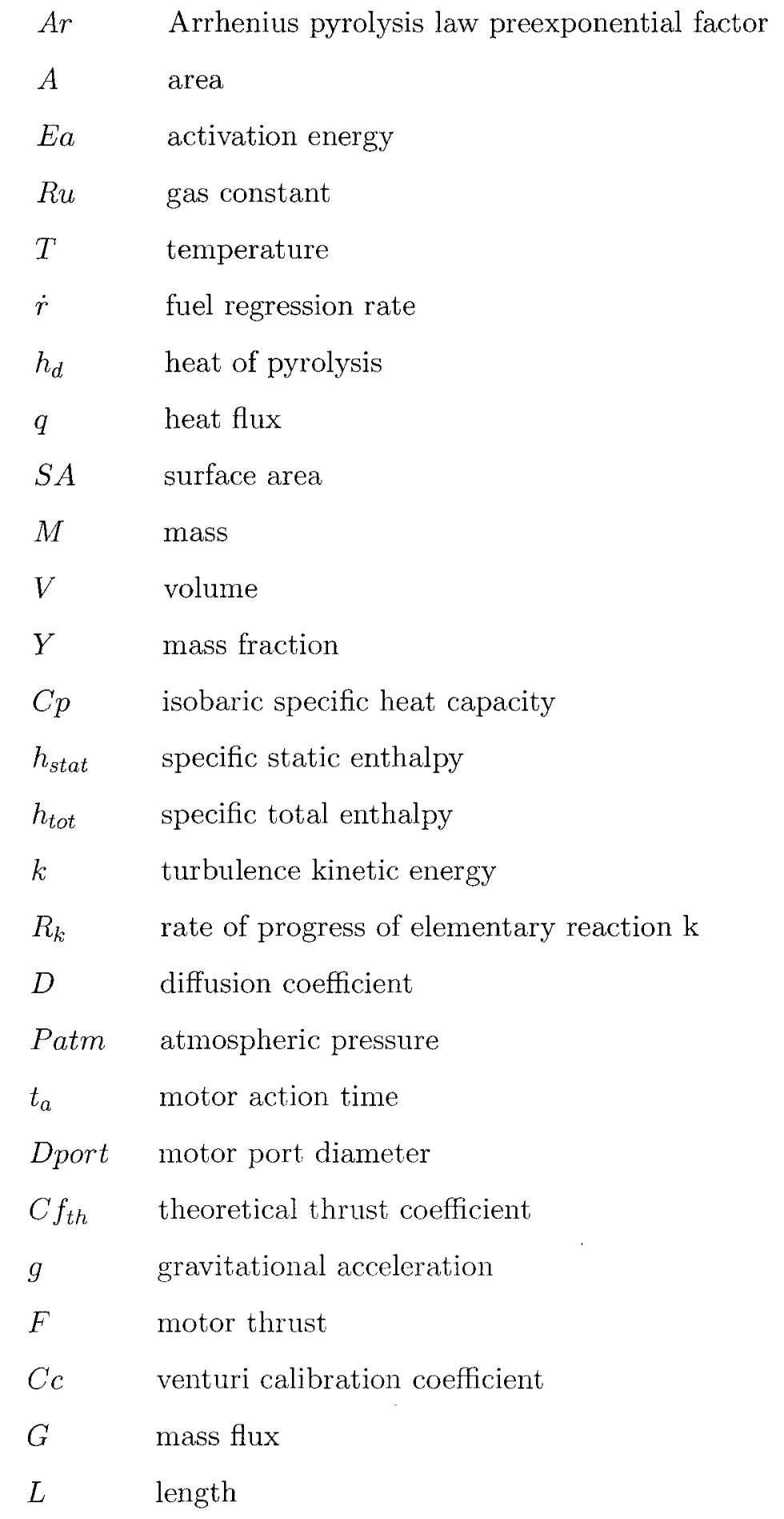

xvii 
$L^{*} \quad$ combustion chamber characteristic length

\section{Subscripts}

$\begin{array}{ll}c & \text { chamber condition } \\ f & \text { fuel, final } \\ o x & \text { oxidizer } \\ s & \text { surface } \\ w & \text { wall } \\ i & \text { mixture component i, initial } \\ r e f & \text { reference quantity } \\ t o t & \text { total quantity } \\ t & \text { turbulent quantity }\end{array}$

xviii 


\section{Chapter 1}

\section{Introduction}

\subsection{Background}

Hybrid rocket propulsion systems offer monetary, safety, and ease of storage advantages over their more traditional solid and bi-propellant counterparts, due to the use of an inert fuel grain that is kept separate from the oxidizer until motor ignition. Additionally, they typically have a higher specific impulse than solid rocket motors, and they can be restarted and throttled. At large scales, hybrid rockets are an unproven technology and have not been employed in commercial space launch applications. They have been used in target missiles and low cost tactical missiles, as well as high-energy upper stage motors. Large hybrid development work was carried out in 1999 when a group of aerospace companies tested several 250000 lbf hydroxyl terminated polybutadiene/liquid oxygen (HTPB/LOX) motors as a part of a feasibility study for strap on boosters for space launch vehicles [1]. The 
most recent example of a hybrid rocket utilized for space launch was the hydroxyl terminated polybutadiene/nitrous oxide system employed on X-Prize winner SpaceShipOne, that was designed and built by SpaceDev Inc. out of Poway, CA.

Hydrogen peroxide (HP) is a storable and nontoxic oxidizer that has been used in torpedoes and turbopumps as a gas generator [2], also in rocket engines for aircraft boost (German Me 163 and US F104) [1], and launchers (British Black Knight). Concentrated hydrogen peroxide has been experimentally investigated within the context of a hybrid rocket by various researchers, Wernimont and Heister[3], Wernimont and Meyer[4], Humble et al.[5], Lund et al.[6], Osmon[7]. The first two investigations utilized polyethylene and $85 \% \mathrm{HP}, 88 \% \mathrm{HP}$ respectively. Additionally, [4] presented data from previous work with HP, specifically one set of data pertaining to hydroxyl terminated polybutadiene (HTPB). The third was research at the USAF Academy to characterize the performance of polyethylene and $85 \%$ HP. The fourth was a team consisting of Lockheed Martin, Thiokol, and Rocketdyne funded by NASA to develop a gas generator fuel for a hybrid rocket to utilize HP as the oxidizer. The last was work out of Northrop Norair to characterize the performance characteristics of $90 \% \mathrm{HP}$ with lithium aluminum hydride.

To the author's knowledge, no previous experimental investigation of the performance characteristics of metallized HTPB fuel with HP oxidizer has been conducted. Likewise, it would appear that a numerical model has not previously 
been constructed to predict the regression rate of an HTPB fuel and hydrogen peroxide propellant combination and compare it directly to experimental data.

The experimental tests reported herein were conducted under the auspices of the Department of National Defence contract "HyperG: Hydro Reactive Gas Generator Hybrids" and performed using equipment and expertise at Cesaroni Technology Incorporated in Gormley, Ontario over the period May-August, 2004. The numerical model was built at Carleton University. It should be noted that despite the title of the HyperG program, no gas generator fuels were used during the experimental testing that is presented in this work.

\subsection{Theory}

The majority of the rocket theory and specifically hybrid rocket motor theory presented in this section is found in [1]. The reader is directed to this reference for details of the results presented here.

A hybrid rocket is traditionally a rocket motor that uses a solid fuel grain with a port through which liquid oxidizer from an injector is flowed. The solid fuel is decomposed and vapourized by heat transfer from the boundary layer to the fuel and diffuses into the boundary layer where it reacts with the oxidizer, which diffuses from the core flow, to produce a diffusion flame. Thus, the fuel flow rate and therefore the oxidizer to fuel ratio (OF) of the motor during operation are coupled to 
the internal aerodynamics of the motor. The reactants are then accelerated through a convergent-divergent nozzle as in traditional rocket motors. Figure 1.1, taken from [1], is a simple schematic of a hybrid rocket motor.

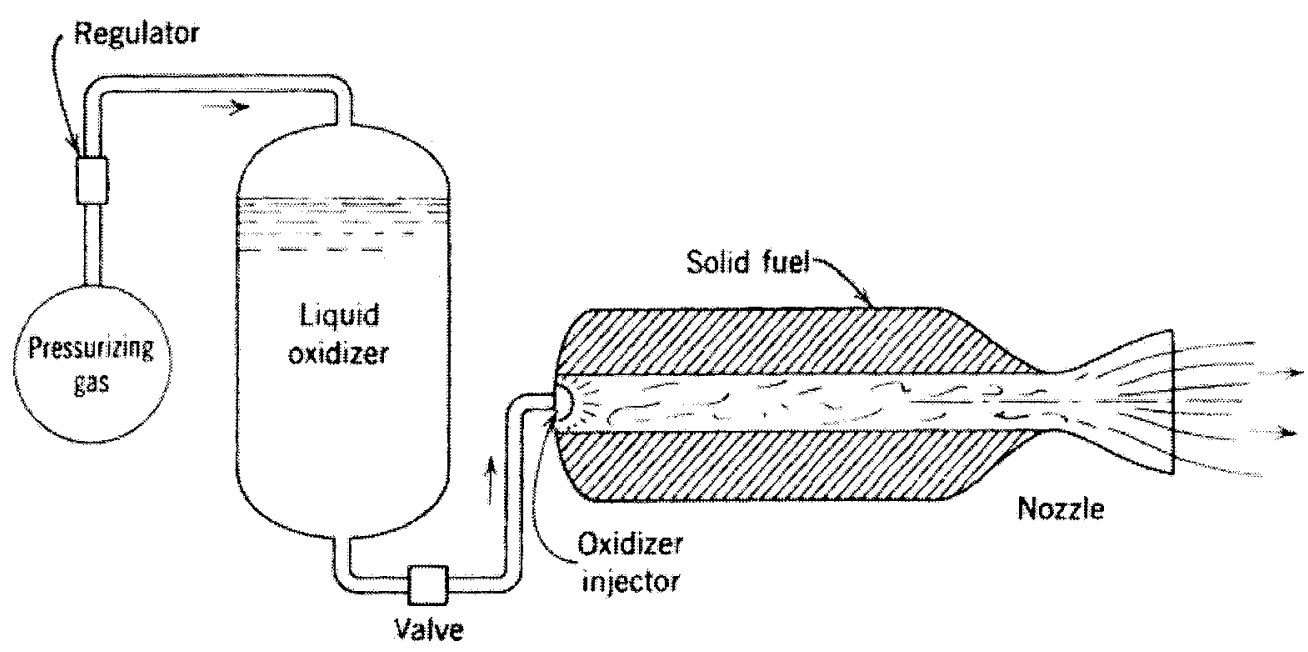

Figure 1.1: Simplified diagram of a hybrid rocket motor[1].

\subsubsection{Rocket theory}

The relations presented in this section are applicable to any device producing thrust by accelerating a gas through a nozzle.

The total impulse of the motor over some time is given as follows.

$$
I_{t o t}=\int_{0}^{t} F d t
$$

Where $\mathrm{F}$ is the motor thrust. A common figure of merit for a rocket propulsion system is the specific impulse, or the thrust per unit weight of propellant. The 
time average value of specific impulse is calculated as follows.

$$
I S P=\frac{\int_{0}^{t} F d t}{g \cdot \int_{0}^{t} \dot{m} d t}
$$

Where $\dot{m}$ is the propellant mass flow rate, $g$ the gravitational acceleration.

The total thrust produced by a rocket is found via the momentum equation:

$$
F=\dot{m} U_{e x i t}+\left(p_{e x i t}-p_{a m b}\right) A_{e x i t}
$$

Where $U_{\text {exit }}$ is the nozzle exit velocity, $p_{\text {exit }}$ the nozzle exit pressure, $p_{a m b}$ the ambient pressure, and $A_{\text {exit }}$ the nozzle exit area. The effective exhaust velocity is defined as:

$$
c=\frac{F}{\dot{m}}=U_{\text {exit }}+\frac{\left(p_{\text {exit }}-p_{a m b .}\right) A_{\text {exit }}}{\dot{m}}
$$

Now, the following assumptions are made.

1. the working fluid is homogeneous,

2. the working fluid is a gas and it behaves as an ideal gas,

3. the rocket chamber and nozzle are adiabatic,

4. the flow is frictionless,

5. there are no shocks or flow discontinuities,

6. the propellant mass flow is constant, 
7. the exhaust velocity is completely axially directed,

8. the gas properties (temperature, pressure, density) and the velocity are uniform in a given cross section,

9. the flow is frozen, that is no chemical reactions occur in the nozzle.

These assumptions permit the use of one-dimensional, isentropic flow relationships in deriving the following formulae. The mass flow through a nozzle with choked flow at the throat is given by the following expression:

$$
\dot{m}=\frac{A_{t} U_{t}}{v_{t}}=A_{t} P_{c} \gamma \frac{\sqrt{\left[\frac{2}{\gamma+1}\right]^{\frac{\gamma+1}{\gamma-1}}}}{\sqrt{\gamma \frac{R u}{M W} T_{c}}}
$$

where MW is the molar mass of the fuel, $\mathrm{P}_{c}$ and $\mathrm{T}_{c}$ the chamber pressure and temperature, $v$ the specific volume, $\gamma$ the ratio of specific heats which is assumed to be constant in this analysis.

The portion of Equation 1.5 that depends only on the thermochemical properties of the propellant and is independent of nozzle characteristics is now defined as the characteristic velocity.

$$
c^{*}=\frac{P_{c} A_{t}}{\dot{m}}=\frac{\sqrt{\gamma \frac{R u}{M W} T_{c}}}{\gamma \sqrt{\frac{2}{\gamma+1}} \frac{\gamma+1}{\gamma-1}}
$$

For an isentropic flow, neglecting changes in potential energy, one can express the nozzle exit velocity, $U_{\text {exit }}$ as follows: 


$$
U_{\text {exit }}=\sqrt{\frac{2 \gamma}{\gamma-1} \frac{R u T_{c}}{M W}\left[1-\left(\frac{p_{e x i t}}{P_{c}}\right)^{\frac{\gamma-1}{\gamma}}\right]+U_{c}^{2}}
$$

where $\mathrm{U}_{c}$ is the velocity of the working fluid in the combustion chamber. It is usually negligibly small relative to the velocity in the nozzle throat and is neglected in the analysis.

Equations 1.7 and 1.5 are then substituted into Equation 1.3. One is then left with the ideal thrust equation:

$$
F=A_{t} P_{c} \sqrt{\frac{2 \gamma^{2}}{\gamma-1}\left(\frac{2}{\gamma+1}\right)^{\frac{\gamma+1}{\gamma-1}}\left[1-\left({\frac{p_{e x i t}}{P_{c}}}^{\gamma-1}\right)\right]}+\left(p_{\text {exit }}-p_{a m b}\right) A_{e x i t}
$$

Dividing Equation 1.8 by the chamber pressure, $\mathrm{P}_{c}$, and the throat area $A_{t}$, the definition of the thrust coefficient, Cf, is obtained.

$$
C f=\sqrt{\frac{2 \gamma^{2}}{\gamma-1}\left(\frac{2}{\gamma+1}\right)^{\frac{\gamma+1}{\gamma-1}}\left[1-\left(\frac{p_{\text {exit }}}{P_{c}}{ }^{\frac{\gamma-1}{\gamma}}\right)\right]}+\frac{\left(p_{\text {exit }}-p_{\text {amb }}\right)}{P_{c}} \frac{A_{\text {exit }}}{A_{t}}
$$

The ideal thrust equation is thus simplified considerably:

$$
F=C f A_{t} P_{c}
$$

Using Equations 1.4, and 1.10, Equation 1.6 can now be written in terms of 
ISP and Cf.

$$
c^{*}=\frac{P_{c} A_{t}}{\dot{m}}=\frac{F}{C f \dot{m}}=\frac{c}{C f}=\frac{I S P \cdot g}{C f}
$$

The figures of merit for the propellant combination $\left(c^{*}\right.$, Equation 1.6) and propellant combination / nozzle performance (ISP, Equation 1.2) were used to gauge the performance of the propellants tested in the HyperG program.

\subsubsection{Hybrid rocket theory}

The fuel regression rate (and hence the OF ratio and total thrust) of a hybrid motor is intrinsically coupled to the internal ballistics of the combustion chamber. Inside the fuel port, a turbulent diffusion flame is formed within a turbulent boundary layer, and the primary mode of heat transfer to the fuel surface is convection.

The majority of analytical work done to characterize the regression rate of hybrid rocket motors was completed by Marxman, Gilbert, Wooldridge, and Muzzy, for example in References [8], [9]. A simplified model of the convective heat transfer in a turbulent boundary layer on a flat plate was applied to the hybrid rocket motor flow field to derive an expression for the fuel regression rate in terms of design parameters. The expression was later modified by the same authors to include the radiative heat transfer contribution from a particle cloud. The analysis is summarized below to provide the origin for the form of the regression rate correlations 
derived from the data in the HyperG tests.

Figure 1.2, adapted from [1], provides the focal point of the discussion. Shown is both a turbulent momentum and thermal boundary layer over a fuel grain surface, the former is assumed to be thicker than the latter. A turbulent diffusion flame is established inside the boundary layer, into which the products diffuse. If one neglects radiation and heat conduction through the fuel mass the resulting surface energy balance is given by:

$$
\dot{Q}_{s}=h \Delta T=\left.\lambda_{g} \frac{\partial T}{\partial y}\right|_{y=0}=\rho_{f} \dot{r} h_{v}
$$

where

$\mathrm{h}$ is the convective heat transfer film coefficient,

$\Delta T$ is the temperature difference between the flame zone and the fuel surface,

$\lambda_{g}$ is the thermal conductivity of the gas phase,

$\rho_{f}$ is the fuel density,

$\dot{r}$ is the fuel surface regression rate,

$h_{v}$, is the overall heat of vapourization of the fuel.

The Reynolds analogy for a turbulent boundary layer over a flat plate is used to relate the skin friction coefficient with blowing, $C_{f}$, to the Stanton number, $C_{h}$ and the Prandtl number, Pr. 
Boundary layer edge

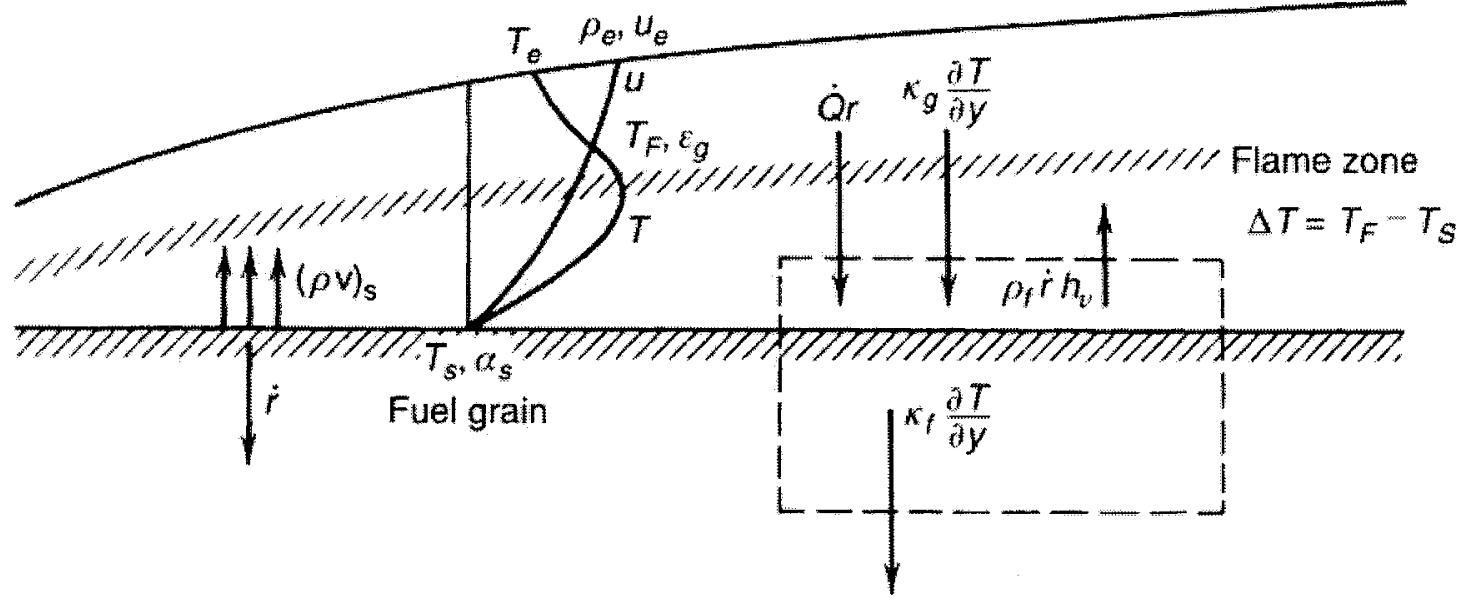

Figure 1.2: Energy balance at the fuel surface in a hybrid rocket motor[1].

$$
C_{h}=\frac{C_{f}}{2} \operatorname{Pr}^{-\frac{2}{3}}
$$

The Stanton number can be written as:

$$
C_{h}=\frac{\left.\lambda_{g} \frac{\partial T}{\partial y}\right|_{y=0}}{\Delta h \rho_{e} U_{e}}
$$

where $\Delta h$ is the change in enthalpy between the flame and fuel surface, and $\rho_{e}$ and $U_{e}$ are the density and velocity at the edge of the boundary layer, which is pure oxidizer.

Substituting Equations 1.14 and 1.13 into Equation 1.12 one obtains: 


$$
\dot{r}=\frac{C_{f}}{2} \frac{\Delta h}{h_{v}} \frac{U_{e} \rho_{e}}{\rho_{f}} \operatorname{Pr}^{-\frac{2}{3}}
$$

The skin friction coefficient without blowing, $C_{f 0}$, can be related to the Reynolds number in a boundary layer without blowing as follows:

$$
\frac{C_{f 0}}{2}=0.0296 R e_{x}^{-0.2}
$$

for $\left(5 \times 10^{5} \leq R e_{x} \leq 1 \times 10^{7}\right)$. Define the blowing coefficient as follows,

$$
\beta=\frac{(\rho v)_{s}}{\rho_{e} U_{e} \frac{C_{f}}{2}}
$$

and note that Sutton[1] has demonstration that following is also true,

$$
\beta=\frac{\Delta h}{h_{v}}
$$

for flows with $\operatorname{Pr}=1.0$.

Workers[10] have developed the following relationship for the effect of blowing on skin friction:

$$
\frac{C_{f}}{C_{f 0}}=1.27 \beta^{-0.77}
$$

for $(5 \leq \beta \leq 100)$. Lastly, note that $\rho_{e} U_{e}$ is the definition of the total mass 
flux through the motor and use Equations 1.19, 1.18 (taking $\operatorname{Pr}=1.0$ ), and 1.16 in Equation 1.15, noting the definition of Reynolds number, $R e_{x}=\frac{\rho_{e} U_{e} x}{\mu_{e}}$.

$$
\dot{r}=0.036 \frac{G_{t o t}^{0.8}}{\rho_{f}}\left(\frac{\mu_{e}}{x}\right)^{0.2} \beta^{0.23}
$$

Note that the coefficient in Equation 1.20 applies for English units only. Equation 1.20 was modified to account for radiative heat flux from a particle cloud in the motor by modifying Equation 1.12 [8]:

$$
\dot{Q}_{s}=\rho_{f} \dot{r} h_{v}=\dot{Q}_{c} e^{-\frac{Q_{r a d}}{Q_{c}}}+\dot{Q_{r a d}}
$$

where the radiative heat flux has the following form,

$$
Q_{\text {rad }}^{\cdot}=\sigma_{f} \alpha T_{\text {flame }}^{4} \epsilon_{g}
$$

where $T_{\text {flame }}$ is the flame temperature, $\alpha_{f}$ the fuel surface absorptivity coefficient, $\sigma$ the Stefan-Boltzmann constant and $\epsilon_{g}$ the emissivity of the particle cloud. The gas emissivity is then assumed to have the following form (this is discussed further in Section 3.4.4):

$$
\epsilon_{g}=1-e^{-A C z}
$$

where $A$ is the particle cloud attenuation coefficient, $C$ the particle cloud 
number density, and $z$ the radiation path length. If one assumes a particle cloud number density proportional to chamber pressure and a path length proportional to port diameter the following functional dependence of the regression rate is obtained.

$$
\dot{r}=\dot{r}\left\{G_{0}, L,\left(1-e^{-\frac{P}{P r e f}}\right),\left(1-e^{-\frac{D}{D_{r e f}}}\right)\right\}
$$

Here $L$ is the length of the port and $P$ and $D$ refer to the motor chamber pressure and port diameter respectively.

In this work and most other experimental work with hybrid motors, all of the functional dependencies of regression rate in Equations 1.20 and 1.24 have been grouped into one constant and a correlation to only total mass flux obtained.

$$
\dot{r}=a G_{t o t}^{n}
$$

Here $a$ and $n$ are constants that are fit to the data obtained from a number of tests for each propellant combination and operating condition. This is the approach that was adopted in this work to search for a correlation for the regression rate data obtained for each propellant combination tested. 


\subsection{Previous experimental studies of hybrid rocket mo- tors}

Due to the lack of published experimental work on the use of HP as an oxidizer with metallized or non-metallized HTPB fuel, a direct comparison with the results obtained for the propellant combinations tested in HyperG program could not be made. The work of Lund et al.[6] was not used for comparison to this work as the fuel in that study was a gas generator which behaves differently than an inert grain. Several sets of experimental results are referred to in this work for comparison to the results presented, and these experiments are summarized in this section. All experimental data used for comparison came from laboratory scale motors which were typically approximately two inches in diameter, or from slab motors with similar port heights. Work with propellant combinations that did not utilize HTPB as the fuel or $\mathrm{HP}$ as the oxidizer were used where necessary for comparison regarding the general behaviour of lab scale hybrid motors.

\section{Wernimont and Heister [3]}

Results from 100 tests spanning a three year study of lab scale hybrid rocket motors utilizing $85 \%$ hydrogen peroxide and polyethylene with an undisclosed "consumable catalytic-ignition device" were presented in this work. Chamber pressures ranged from 100 psia - 400 psia and initial oxidizer fluxes ranged from $0.1 \mathrm{lbm} / \mathrm{in}^{2} \mathrm{~s}-1.2$ 
$\mathrm{lbm} / \mathrm{in}^{2} \mathrm{~s}$. The experimental apparatus and materials of construction were very similar to those of the HyperG experiments. The mass flow was controlled only by pressure differential between combustion chamber and oxidizer tank, no cavitating venturi was used. The authors found a reduced dependence of regression rate on port mass flux in the range $0.1 \mathrm{lbm} / \mathrm{in}^{2} \mathrm{~s}-0.3 \mathrm{lbm} / \mathrm{in}^{2} \mathrm{~s}$ with chamber pressures of 200 psia - 400 psia. At the lower pressures tested, the spatially and temporally averaged regression rates showed the classical turbulent convection dominant behavior with a correlation exponent of 0.8 on the mass flux. Combustion efficiency was shown to increase with increasing motor characteristic length $\left(\mathrm{L}^{*}\right)$. No significant combustion instability was noted.

\section{Wernimont and Meyer [4]}

The purpose of the work was to characterize the combustion efficiency, regression rate, and ignition behaviour of a hybrid rocket motor operating with polyethylene solid fuel and $88 \%$ hydrogen peroxide oxidizer. A pellet catalytic bed was used that was made of refractory cement and potassium permanganate. The lab scale motor that was used had a $2 \mathrm{in}$. port diameter. Various decomposition methods for high test hydrogen peroxide were discussed. A summary of prior work for hydrogen peroxide combustion devices was presented, including both experimental data sets and the regression rate correlations deduced from them. Combustion efficiencies 
ranging from $87 \%-98 \%$ were obtained. Spatial and time averaged regression rates ranging from $0.0144 \mathrm{in} / \mathrm{s}-0.0371 \mathrm{in} / \mathrm{s}$ were obtained. A correlation for the regression rate as a function of mass flux with an exponent of 0.8 was obtained but the authors note that the limited amount of data makes this a loose correlation. Stable ignition and combustion were demonstrated.

\section{Osmon [7]}

A shortfall of hybrid rockets is their inability to maintain constant, optimum OF ratios as they are throttled. For this reason it is desirable to use a propellant combination that has a large regression rate dependency on pressure, as opposed to mass flux. A propellant formulation that has solid or liquid particles in the combustion products would theoretically have a regression rate that is largely dependent on pressure, as radiative heat transfer would play a large role in the heating of the fuel surface. Osmon experimentally determined the performance characteristics of $95 \%$ $\mathrm{LiAlH}_{4}$ (lithium aluminum hydride), $5 \%$ polyethylene with $90 \%$ hydrogen peroxide by weight, a combination that had a theoretical peak ISP of $287 \mathrm{~s}$. It was noted that since $\mathrm{LiAlH}_{4}$ starts to decompose at $125^{\circ} \mathrm{C}$ and reacts with trace amounts of water, large scale manufacture of the fuel grains would pose a serious difficulty. The experiments were carried out at Edwards Air Force Base using lab scale motor hardware with a grain length of 20 in., inner diameter of $1.75 \mathrm{in}$. and web thickness 
of $0.5 \mathrm{in}$. Spontaneous ignition of the $\mathrm{LiAlH}_{4}$ fuel grain with the decomposed HP occurred. Chamber pressures ranged from 120 psia - 404 psia. Low frequency, low amplitude oscillations were observed which were attributed to periodic blowing of a blanket of molten aluminum from the fuel surface (aluminum accounted for $67.5 \%$ by weight of the fuel).

Combustion efficiencies ranged from $78.5 \%-86.0 \%$ and ISP efficiencies from $76.7 \%-79.7 \%$. These relatively low numbers were attributed to: a) lower efficiency predictions for small scale engines having high concentrations of particles in the exhaust products and b) large particles of liquid aluminum oxide leaving the fuel grain, forming a protective oxide coating and having a core that had not reached the boiling temperature of aluminum, thus having no driving force to break through the oxide coating and continue combustion of the aluminum fuel. It was noted that the addition of magnesium to the fuel should both increase the flame temperature and allow more complete burning of the fuel due to the fact that magnesium burns in the vapor phase, while aluminum has a surface reaction. Also, magnesium has a much lower boiling temperature than that of aluminum. A turbulator was employed just downstream of the fuel grain in an attempt to improve the combustion efficiencies by increasing mixing and residence time in the motor. It had no effect, further substantiating the hypothesis that the low chamber temperature was responsible for the low combustion efficiencies. An empirical correlation for the temporal and 
spatially averaged regression rate as a function of oxidizer mass flux was obtained that had both a oxidizer flux and pressure exponent of 0.4 .

\section{Lips $[11],[12]$}

The four propellant combinations tested in this work were $60 \% \mathrm{Al} / 40 \%$ polyurethane(PU) with FLOX $-40^{1}, 60 \mathrm{w} \% \mathrm{Al} / 40 \% \mathrm{PU}$ with liquid oxygen (LOX), $80 \% \mathrm{Al} / 20 \% \mathrm{PU}$ with FLOX-90 and $80 \% \mathrm{Al} / 20 \%$ carboxyl terminated polybutadiene (CTPB) with FLOX-90. All percentages are given by weight. The tests were conducted using a vertical thrust stand. The motor assembly included a mixing diaphragm, reaction chamber, nozzle, injector and splash-block. The vortex-barrier film injector was optimized through several test firings. A tungsten carbide/graphite mixing diaphragm was added downstream of the fuel grain to improve the mixing of the stratified oxidizer and fuel flows. A graphite-lined, porous carbon insulated and ablatively cooled reaction chamber was used to increase the characteristic length and particle residence time in the motor. The nozzle insert was constructed of a high density, fine grade graphite material with high thermal conductivity. The nozzle support section was constructed of an asbestos-phenolic molding compound material and a carbon-phenolic barrier material was applied to the interface between the graphite nozzle insert and the nozzle support material. This barrier served as an insulator which maintained a high enough nozzle throat temperature to prevent

\footnotetext{
${ }^{1}$ FLOX-40 is $40 \%$ fluorine and $60 \%$ oxygen by weight
} 
condensation of aluminum deposits.

The total mass flux range investigated was $2 \mathrm{~g} / \mathrm{cm}^{2} \mathrm{~s}-10 \mathrm{~g} / \mathrm{cm}^{2} \mathrm{~s}$. The chamber pressure for each test was approximately 10 bar. Local regression rates were determined from measurements at ten locations along the length of the fuel grain. The average regression rate in the aft one third of the fuel grain was correlated with oxidizer mass flux averaged over the initial and final port diameters, yielding a correlation exponent of 0.5 on the oxidizer mass flux for both $\mathrm{Al} / \mathrm{PU} / \mathrm{FLOX}$ propellant combinations. The regression rate of the $80 \% \mathrm{Al} / 20 \% \mathrm{PU}$ with FLOX-90 was approximately $33 \%$ higher than that of the $60 \% \mathrm{Al} / 40 \% \mathrm{PU}$ with FLOX-40. The correlation of regression rate with total mass flux yielded the same correlation exponent. Due to the sensitivity of $c^{*}$ calculations to small changes in throat diameter in lab scale motor firings, thrust measurements were used to estimate $c^{*}$ in the majority of the cases.

It was found that firings without the mixing diaphragm resulted in extreme nozzle erosion and low combustion efficiency. Increasing the characteristic length of the reaction chamber resulted in an increase in combustion efficiency, indicating that the majority of aluminum particle combustion occurred in the reaction chamber. The combustion efficiencies obtained in this experiment ranged from 0.78-0.97, with combustion efficiency increasing with increasing fluorine content in the oxidizer. Although the combustion efficiency did not differ between the CTPB and PU binders 
in the $80 \% \mathrm{Al}$ loaded grains with FLOX-90, a low frequency "chuffing" instability was observed in the CTPB system. The $80 \% \mathrm{Al} / 20 \% \mathrm{PU}$ surfaces were clean after firing, while the CTPB surfaces were covered with a dark brown char layer. Overall it was determined that increasing the aluminum loading in the fuel grain increased the regression rate but decreased combustion efficiency.

\section{Strand and Ray [13],[14]}

The lab scale tests carried out in this work used a windowed slab motor geometry and R45M HTPB (fuel) and GOX (oxidizer). Chamber pressures ranged from 140 psia - 280 psia and head end oxidizer mass fluxes ranged from $0.01 \mathrm{lbm} / \mathrm{in}^{2} \mathrm{~s}-0.1$ $\mathrm{lbm} / \mathrm{in}^{2} \mathrm{~s}$. The turbulence level in cold flow conditions duplicating the oxygen mass flows and chamber pressures at test conditions was measured through a spark plug igniter port using a hot wire anemometer. The amplitude of the core flow turbulence was approximately $10 \%$ of the mean velocity in the cold flow simulation tests; this result is obviously strongly injector dependent. A correlation of regression rate with oxidizer mass flux yielded a power law curve fit with an exponent of unity, whereas a curve fit with the total mass flux yielded the near 0.8 power law dependence predicted by turbulent boundary layer theory. The regression rate appeared independent of pressure, although for the lower test pressures the oxidizer mass flux dependence diminished at higher oxidizer mass flux. This would indicate that the 
chemical kinetics was the dominant process in this region. The axial dependence of regression rate was minimal except at the one quarter length position which the authors state was likely due to "turbulent mixing enhancement resulting from vortices shed from the slab forward face". Combustion instability at approximately 3-4 $\mathrm{Hz}$ was observed and attributed to what the authors state was "some type of flow-combustion turbulence interaction along the surface of the fuel slab."

An analytical model for regression rate dependency was developed based on the experiments and was fit to the data for some $40 \% \mathrm{Al} / 30 \%$ coal $/ 30 \% \mathrm{HTPB}$ tests. The observed power law dependence of regression rate on total mass flux was lower than 0.8 for this propellant, indicating the strong effect of radiation on the regression of the metallized fuel, especially at lower values of mass flux. The model predicted a reduced pressure sensitivity with increasing mass flux and under-predicted the pressure dependence at lower mass flux, likely due to decreased efficiency of aluminum combustion at lower mass flux which would have in turn reduced the number density of oxide smoke and therefore the radiative component of fuel surface heat transfer. The pressure dependence in the model was manifested through the radiation terms, and as such data for conditions theorized to lead to a kinetics related pressure dependence (high mass flux, low pressure) were omitted. 


\section{Smoot and Price, non-metallized fuel [15]}

Three different binder compounds (butyl rubber, polybutadiene-acrylic acid copolymer (PBAA), polyurethane) were combusted in a lab scale slab burner with oxidizers ranging from $0 \%-100 \%$ fluorine in oxygen, by weight. The tested chamber pressure range was 20 psia-160 psia, and oxidizer flux range was $0.014 \mathrm{lbm} / \mathrm{in}^{s} \mathrm{~S}-0.170$ $\mathrm{lbm} / \mathrm{in}^{s} \mathrm{~s}$. Regression rates were averaged over the burn time and grain length, and obtained by measuring the grain height at five positions along the length. Regression rate was found to decrease with decreasing fluorine content of the oxidizer and follow the theoretically predicted 0.8 power law relationship with total mass flux in the low mass flux regions $\left(<0.07 \mathrm{lbm} / \mathrm{in}^{s} \mathrm{~s}\right)$. It was found that at high flow rates the regression rate became independent of flow rate, especially at lower pressures. There was little effect of the binder composition on regression rate when tested with the same oxidizers. The regression rate in the pressure independent region of data was found to agree well with flat plate turbulent boundary layer and fully developed duct flow correlations. The authors attributed the pressure dependency of the propellant formulations to "heterogenous attack of active oxidizer on the fuel binder" at high mass flow rates. Varying the oxidizer composition had little effect on the observed pressure dependence. 


\section{Smoot and Price, metallized fuel [16]}

The effects of pressure, oxidizer flow, oxidizer composition and fuel grain composition on the lithium hydride(LiH)/butyl rubber/FLOX system were examined using a slab burner configuration. The tested chamber pressure range was 20 psia-160 psia, and oxidizer flux range was $0.014 \mathrm{lbm} / \mathrm{in}^{2} \mathrm{~s}-0.160 \mathrm{lbm} / \mathrm{in}^{2} \mathrm{~s}$. The following conclusions were drawn from the experimental data. Increasing the $\mathrm{LiH}$ loading in the fuel from $0 \%-50 \%$ by weight at low mass flux conditions had little effect on regression rate however further increases from 50\%-90\% had a substantial effect. At higher mass flux, regression rate increases were produced over the entire range of $\mathrm{LiH}$ loading. At low mass flux the regression rate showed the traditional power law dependency on mass flux with an exponent of 0.8 , however at high mass flux the regression rate exhibited a definite dependence on pressure. An increase in LiH loading reduced the observed pressure dependence in the high mass flux region. The classical regression rate models based on turbulent heat transfer predicted the regression rate well in the low mass flux region, however not in the high mass flux, pressure dependent region. The authors state that, "The most likely cause of this observed pressure dependence is a rate-limiting kinetic reaction, possibly heterogeneous in nature." 


\section{Chiaverini et al. [17]}

The regression rate characteristics of HTPB solid fuel burning with GOX were determined experimentally using windowed slab geometry motors. The following parameters were measured: instantaneous and average solid fuel regression rates under varying operating conditions including GOX flux level, motor pressure and fuel formulation, solid fuel surface temperatures and sub-surface thermal wave profiles as a function of operating conditions, and the effects of solid fuel additives on regression rate behaviour. An Arrhenius rate expression to relate regression rate to surface temperature was developed and empirical correlations to relate the regression rate to operating conditions and geometrical parameters were deduced. It was noted that the addition of carbon black to the fuel had no noticeable effect on either regression rate or surface temperature. A critical flow venturi with adjustable throat area was used to maintain a steady mass flow of GOX through the main line. The diagnostics were fine wire thermocouples, pressure transducers, an x-ray radiography system and an ultrasonic transducer. Thermocouples were embedded in the fuel during casting to measuring the sub-surface fuel temperature profile during the test. Erosion of the graphite nozzle caused a chamber pressure drop during the tests.

Deviation of the mass flux exponent from the value of 0.8 derived for turbulent flow over a flat plate indicated the importance of non-convective process 
such as chemical kinetics and radiative heat transfer on solid fuel regression rates in hybrid motors. Other important parameters such as oxidizer to fuel ratio, motor pressure, axial grain location, and variation of port diameter were not included in the correlation. Regression rates were shown to increase with downstream motor distance after an initial region of decrease. This was due to the increase of mass flux due to fuel addition. The regression rate correlation indicated that in the low mass flux region $\left(<140 \mathrm{~kg} / \mathrm{m}^{2} \mathrm{~s}\right)$ the regression behaviour diverged from the convective solution. It was surmised that this was due to radiant heat flux from sources such as soot and gas-phase combustion products to the fuel. An increase in the regression rate at higher chamber pressures was observed in the lower mass flux regime where convection was less dominant. The effect of finite rate reaction kinetics which may act to reduce the regression rate at very high mass fluxes, was not observed in the investigation.

The addition of UFAL (ultra-fine aluminum powder) resulted in a regression rate increase of approximately $40 \%$ over that measured during the baseline HTPB tests. A higher surface temperature and thinner thermal wave was observed in the solid fuel at downstream locations relative to upstream locations. Additionally, it was observed that higher mass flux had the effect of increasing surface temperature and reducing thermal wave thickness. The fuel surface temperature ranged from $930 \mathrm{~K}$ to $1190 \mathrm{~K}$ depending on operating conditions. The relatively low activation 
energies measured in the study indicated that the overall regression process was limited by physical desorption of high molecular weight fuel fragments from the surface rather than by bulk phase chemical kinetics.

\subsection{Previous numerical studies of hybrid rocket motors}

Although no references to a numerical model using a HTPB/HP propellant combination in a hybrid rocket configuration were found, several numerical studies of HTPB/GOX or HTPB/LOX hybrid motors were located. A summary is presented below of the studies most relevant to this work. The study by Serin and Gogus provided the most useful information as it was carried out using a commercial CFD code. The general method described in that study of interfacing an external code to calculate regression rate to the CFD software was also used here.

\section{Serin and Gogus [18]}

A commercial CFD software package, CFD-ACE, was used to model a HTPB/GOX slab geometry hybrid motor. A FORTRAN code was used to compute the fuel surface temperature and regression rate interactively with CFD-ACE. Results for regression rate were compared with theoretical correlations and experimental results from Penn State High Pressure Combustion Laboratory using a slab motor geometry. The oxygen mass flow in the specific test chosen for the model was $0.108 \mathrm{~kg} / \mathrm{s}$ with maximum chamber pressure of 34.5 bar. A steady state solution was obtained using 
the $\mathrm{k}-\varepsilon$ turbulence, and equilibrium reaction combustion models in CFD-ACE. A structured, single-domain, body-fitted, 2-D cartesian grid system with 10500 cells was used. The fuel injection velocity and surface temperature were specified as boundary conditions in ACE, and were computed iteratively in the FORTRAN code for ten regions along the length of the fuel. The flame height was given by the vertical location of maximum temperature at each location above the fuel wall. The radiative heat flux contribution from the $\mathrm{H}_{2} \mathrm{O}$ and $\mathrm{CO}_{2}$ constituents in the chamber was calculated using the method presented in [19], and the $\mathrm{CO}$ radiation contribution was ignored. An Arrhenius type relation was employed for closure of the energy balance equation to describe temperature dependence of HTPB regression rate and the product of HTPB ablation was assumed to be 1,3-butadiene.

The results of the FORTRAN code became the input for surface temperature and mass injection at the boundaries in CFD-ACE. The CFD-ACE solution was then obtained, and the procedure repeated until the surface temperature ceased to differ between successive runs by more than $10 \mathrm{~K}$. Steady state solutions were obtained for motor conditions corresponding to those measured at the start and end of the test, and the channel and nozzle geometry were varied in the model. It was found that the fuel regression rate varied axially along the duct, and that numerical results agreed well with empirical correlations obtained at Thiokol Corp. that neglected any regression rate dependency on axial coordinate. The regression rate data for 
the experimental motor from which the numerical motor was modeled were not presented in the paper.

\section{Cheng, Farmer, Jones and McFarlane [20]}

A numerical analysis of a hybrid motor utilizing HTPB fuel and LOX was performed, and the results were compared to the $\mathrm{AMROC}^{2} \mathrm{DM}-1250 \mathrm{klbf}$ hybrid motor that was first tested in 1993 . Turbulence was modeled with a temperature modified $k-\varepsilon$ model, and the combustion process was modeled using a simplified chemical kinetics model with the product of the HTPB pyrolysis assumed to be 1,3-butadiene. Both axisymmetric and 3-D motor configurations were simulated at steady state with a solver called the FDNS code, a mature Navier-Stokes solver. The code had the capability of treating the fuel surface pyrolysis as an internal boundary condition, allowing pyrolysis to be modeled as a function of heat load while still solving the energy equation in the fuel grain numerically. Sub-models were used within the FDNS code to describe LOX vapourization, fuel pyrolysis and radiation. The fuel pyrolysis was modeled by balancing the convective and radiative heat transfer at the wall with the heat of pyrolysis of the HTPB. The radiative heat transfer was approximated using an effective gas emissivity of 0.55 and an average emission temperature between the adiabatic flame and pyrolysis temperature.

\footnotetext{
${ }^{2}$ The American Rocket Company was founded in 1985 and existed for eight years. AMROC completed approximately 300 hybrid motor tests with thrust ranges from $100-250000 \mathrm{lb}$. SpaceDev acquired exclusive rights to a large quantity of AMROC data and intellectual property.
} 
The chamber pressure and thrust for a given configuration were calculated using a specified oxidizer mass flux and fuel regression rate to compare with measured AMROC data. The comparison showed that the three dimensional model over-predicted the measured specific impulse because the combustion model did not include radical formation and therefore over predicted the heat release of the reaction.

\section{Merkle and Venkateswaran [21]}

A numerical study of lab scale and large scale hybrid rocket motors was performed using data from Chiaverini et al.[22],[23] for validation. The computational model was subsequently used to predict the effects of size scale-up from lab scale to full size motor, and the effect of going from a planar (slab motor) to axisymmetric configu-

ration. A two step reaction mechanism was employed, assuming the only product of pyrolysis of $\mathrm{HTPB}$ to be 1,3-butadiene. An Arrhenius rate equation for the HTPB pyrolysis was used to close the energy balance at the solid-fuel interface. The $k-\varepsilon$ model was used for turbulence modeling and the radiative heat transfer was modeled using a simplified emissions model that was calibrated using the experimental data mentioned in the references given above. In many of the simulations the computational domain included only the straight section of the motor geometry; the nozzle and reaction chamber sections were omitted. The flow field was assumed to 
be quasi-steady at each stage in the burn and thus the time independent form of the governing equations was solved.

The authors state that the results of the model indicated that, "The strong acceleration arising from the thermal energy release and mass addition along with the depletion of oxidizer on the centerline causes the boundary layer theory which has been frequently used to represent the hybrid combustion process to become quite approximate." Fuel surface temperatures were measured to be $900 \mathrm{~K}-1000 \mathrm{~K}$ in the experiments and were predicted well by the numerical model. The results of the numerical solution of the scaled-up motor geometry showed that the regression rate was dependent not only on the flow rate parameters but also on the geometry of the motor (port shape). This indicated that boundary layer theory could predict qualitative trends in a given configuration but could not account for the effects of port shape. It was shown that the flame was nearer to the wall in non-dimensional terms in large motors; so that non-dimensional flame location did not scale geometrically with motor size. The results showed that the convective heat flux decreased with increasing motor size, while the radiative heat flux increased with increasing motor size. 


\section{Loh and Golafshani [24]}

A numerical solution of the flow field in a HTPB/GOX hybrid rocket motor is presented that was obtained assuming that the flow field had an axisymmetric character. An implicit finite-volume numerical scheme with successive over-relaxation was used to solve the Navier-Stokes equations. Turbulence was modeled using the Baldwin-Lomax algebraic eddy viscosity model, and the combustion process was modeled using a one-step finite rate chemistry model. The fuel regression rate at the surface was calculated using an energy balance and the radiative heat flux to the fuel surface was neglected. Solutions for both laminar and turbulent conditions were calculated to show the difference in the resulting regression rates and chamber pressures. Results were compared to experimental data in a limited fashion, although the source of this data was not given.

\subsection{Summary}

As neither Al/HTPB or AlMg/HTPB fuels have been previously tested with HP in a hybrid motor configuration on a laboratory scale, the experimental portion of this work sought to characterize the performance of these two fuels with HP. The results were compared to data from the literature for various HP/fuel combinations, as well as other highly metallized HTPB fuels with various oxidizers.

A numerical model of the experimental hardware was constructed using 
methods reviewed in the literature, specifically in the work of Serin and Gogus.

The results of the model were compared directly with the experimental data from the HP/HTPB propellant combination. 


\section{Chapter 2}

\section{Experimental Program}

\section{$2.1 \quad$ Program objectives}

The two important performance characteristics for any rocket propulsion system are specific impulse and combustion efficiency. Specific to hybrid propulsion systems is the desire to measure the regression rate of the solid fuel, allowing the determination of the average fuel mass flow rate, overall OF ratio, and the correlation of regression rate with operating parameters. As presented in Section 1.2, turbulent boundary layer theory predicts a power law type variation of regression rate with total mass

flux: $\dot{r} \sim G_{t o t}^{0.8}$. The effect of pressure on the regression rate, whether it be due to the magnitude of the radiative heat flux contribution to the wall heat flux, or pressure dependent chemical kinetics, is manifested as a reduction in this exponent. The tests performed in the experimental program were designed to meet the following objectives. For a lab scale motor using a silver plated nickel screen catalyst pack, 
1. characterize the combustion efficiency of $90 \%$ HP with HTPB, HTPB/aluminum, and HTPB/aluminum/magnesium fuels;

2. characterize the ISP range at sea level of $90 \% \mathrm{HP}$ with HTPB, HTPB/aluminum, and HTPB/aluminum/magnesium fuels;

3. characterize the regression rate range of $90 \% \mathrm{HP}$ with $\mathrm{HTPB}, \mathrm{HTPB} /$ aluminum, and HTPB/aluminum/magnesium fuels;

4. examine the effect of chamber pressure on the combustion efficiency and regression rate;

5. examine the effect of OF ratio on the combustion efficiency;

6. examine the effect of metal loading and metal composition on combustion efficiency and regression rate;

7. develop a regression rate correlation for $90 \% \mathrm{HP}$ with $\mathrm{HTPB} /$ aluminum and HTPB/aluminum/magnesium fuels;

8. to observe and explain any combustion instabilities that arise. 


\subsubsection{Test matrix}

A test matrix was devised to characterize the performance of the following propellant combinations: Al/HTPB/HP, AlMg/HTPB/HP and non-metallized HTPB. Various OF ratios and chamber pressures were tested. The test matrix is included in Figure 2.1 .

The test matrix was constructed as follows. A desired OF ratio and oxidizer mass flux was chosen. An approximate fuel regression rate and available fuel grain length was used to set the required oxidizer flow rate and port diameter of the fuel grain. A chamber pressure was chosen, which, along with the total mass flow, was used to set the nozzle throat diameter. The nozzle throat diameter was limited to approximately $8 \mathrm{~mm}$ to reduce the risk of throat blockage with any debris during the test. Finally, this limit combined with the required oxidizer mass flow set the nitrogen tank feed pressure.

In order to fulfil the test objectives outlined previously, the entries in the test matrix cover chamber pressure ranges from low (200 psi) to high (900 psi), OF ratios for fuel rich, stoichiometric, and oxidizer rich conditions, and total mass flux values from low $\left(60 \mathrm{~kg} / \mathrm{m}^{2} \mathrm{~s}\right)$ to high $\left(300 \mathrm{~kg} / \mathrm{m}^{2} \mathrm{~s}\right)$.

Note that due to very low combustion efficiencies measured in Tests 1 and 2 as well as a large amount of nozzle slag agglomeration and particulate content in the exhaust, all subsequent tests utilized reaction chambers. This is discussed further 


\begin{tabular}{|c|c|c|c|c|c|c|c|c|c|c|c|c|c|}
\hline \multirow[b]{2}{*}{ Commlete } & \multicolumn{13}{|c|}{ Monoprop. } \\
\hline & Test & Fuel & $\begin{array}{l}\text { Pc } \\
\text { (psi) }\end{array}$ & $\begin{array}{l}\text { Po } \\
\text { (psi) } \\
\end{array}$ & $\begin{array}{l}\text { nozzle } \\
\text { (dtide, } \mathrm{mm} \text { ) }\end{array}$ & $\begin{array}{l}\text { pre-comb. } \\
\text { (mm) }\end{array}$ & $\begin{array}{l}\text { post-comb. } \\
\text { (mmm) }\end{array}$ & (mrain & casing & $\begin{array}{l}\operatorname{mox} \\
\text { [kg/s] }\end{array}$ & $\begin{array}{l}\text { port atia. } \\
\text { (mnn) }\end{array}$ & $\begin{array}{l}\text { Gox } \\
\left(\mathrm{kg} / \mathrm{sim}^{\star} 2\right)\end{array}$ & $\begin{array}{l}\text { off } \\
\text { approx. }\end{array}$ \\
\hline \multirow[t]{2}{*}{$\gamma$} & 0 & $\mathrm{H} 2 \mathrm{O}_{2}$ & 110 & 400 & 91763 & nia & nia & nia & 49 & 0.074 & nia & nia & nia \\
\hline & \multicolumn{13}{|l|}{ Hubrid } \\
\hline$Y$ & 1 & 60AI4OHTPB & 630 & 1400 & 9.47 .63 & 36.9 & 0 & 400 & 49 & 0.11 & 25.40 & 133.91 & 2.4 \\
\hline$Y$ & 2 & 60AI40HTPE & 630 & 1400 & 9.47 .63 & 36.9 & 0 & 400 & $4 g$ & 0.11 & 25.40 & 133.91 & 2.4 \\
\hline$Y$ & 3 & 60, $140 \mathrm{HTPB}$ & 630 & 1400 & $9 M 7.63$ & 36.9 & 134.8 & 400 & 59 & 0.11 & 25.40 & 133.91 & 2.4 \\
\hline$Y$ & 4 & ВОД140HТPE & 630 & 1400 & $9 / 23.1$ & 36.9 & 134.8 & 400 & $5 g$ & 0.11 & 31.75 & 85.70 & 2.4 \\
\hline$Y$ & 5 & $60, \mathrm{~A} / 40 \mathrm{HTPB}$ & 225 & 1400 & $15 / 25.1$ & 36.9 & 134.8 & 400 & $5 \mathrm{~g}$ & 0.11 & 25.40 & 133.91 & 2.4 \\
\hline$Y$ & 6 & 60.8140HTPB & 570 & 800 & $9 / 23.1$ & 36.9 & 134.6 & 400 & $5 g$ & 0.08 & 25.40 & 97.39 & 1.3 \\
\hline $\mathrm{Y}$ & 7 & 60A/4OHTPE & 940 & 1400 & $10.5 r 32$ & 36.9 & 134.8 & 400 & $5 g$ & 0.25 & 25.40 & 304.34 & 4 \\
\hline 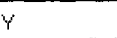 & 8 & ED,, $140 \mathrm{HTPB}$ & 940 & 1400 & $10.5 / 32$ & 36.9 & 134.8 & 400 & 59 & 0.25 & 31.75 & 19478 & 4 \\
\hline$v$ & 9 & 60ANMIG4OHTPB & 600 & 1400 & $9 / 32.1$ & 36.9 & 134.8 & 400 & 59 & 0.11 & 31.75 & 85.70 & 2.2 \\
\hline$Y$ & 10 & 60Allg4OHTPB & 600 & 1400 & $9 / 32.1$ & 36.9 & 134.8 & 400 & $5 \mathrm{~g}$ & 0.11 & 25.40 & 133.91 & 2.2 \\
\hline$Y$ & 11 & 60AMMG 4OHTPE & 930 & 1400 & $10.5 B 32$ & 36.9 & 134.8 & 400 & $5 \mathrm{~g}$ & 0.25 & 31.75 & 194.78 & 4 \\
\hline Y & 12 & 60A.Ping 40HTPE & 930 & 1400 & $10.5 / 32$ & 36.9 & 134.8 & 400 & $5 \mathrm{~g}$ & 025 & 25.40 & 304.34 & 4 \\
\hline$\gamma$ & 13 & 60A & 220 & 1400 & $15 / 26.1$ & 36.9 & 134.8 & 400 & 59 & 0.11 & 25.40 & 133.91 & 2.2 \\
\hline$Y$ & 14 & HTPB & 620 & 1400 & $8 / 20.1$ & 36.9 & 134.8 & 400 & 59 & 0.11 & 25.40 & 133.91 & 6 \\
\hline$Y$ & 15 & HTPE & 620 & 1400 & $8 / 20.1$ & 36.9 & 134.8 & 400 & $5 g$ & 0.11 & 38.10 & 59.52 & 6 \\
\hline
\end{tabular}

Figure 2.1: Test matrix for the HyperG program

in 2.7.7.

\subsection{Theoretical performance calculations}

The program PROPEP [25] was used to determine the stoichiometric OF ratio for each of the three propellant combinations tested. The PROPEP code works by minimizing the Gibbs free energy of the system and solving mass conservation and energy conservation to determine the gas composition, temperature and gas properties at equilibrium. From these values, the ideal characteristic velocity and ideal ISP of the propellant are calculated assuming the working fluid is an ideal gas. PROPEP was also used to determine the theoretical or adiabatic flame temperature, theoretical 
ISP and theoretical characteristic velocity $\left(c^{*}\right)$ for each test at the experimental OF and chamber pressure, in order to compute the combustion efficiency and deviation of ISP from optimal during the data analysis. In all cases the conservative values of theoretical $\mathrm{c}^{*}$ and ISP were calculated assuming frozen flow through the nozzle.

The specifications for Figures 2.2 and 2.3 are: $P_{c}=1000$ psi, reactants originally at $298 \mathrm{~K}$, expansion to Patm $=14.7 \mathrm{psi}$, frozen flow. Note that in all theoretical calculations using PROPEP minor propellant constituents (carbon black, curing agent, etc) were neglected. All fuel metal loading was at $60 \%$ by weight as this delivered the maximum theoretical performance with $90 \% \mathrm{HP}$.

From this figure the following approximate stoichiometric OF ratios were obtained.

HTPB/90\%HP: 6.0

$60 \% \mathrm{Al} / 40 \%$ HTPB $/ 90 \%$ HP: $1.25-2.0$

$60 \% \mathrm{AlMg} / 40 \% \mathrm{HTPB} / 90 \% \mathrm{HP}: 1.75-2.5$

Note that a motor operating at a stoichiometric OF ratio is not necessarily obtaining the highest combustion efficiency, as will be discussed in Section 2.7.7. 


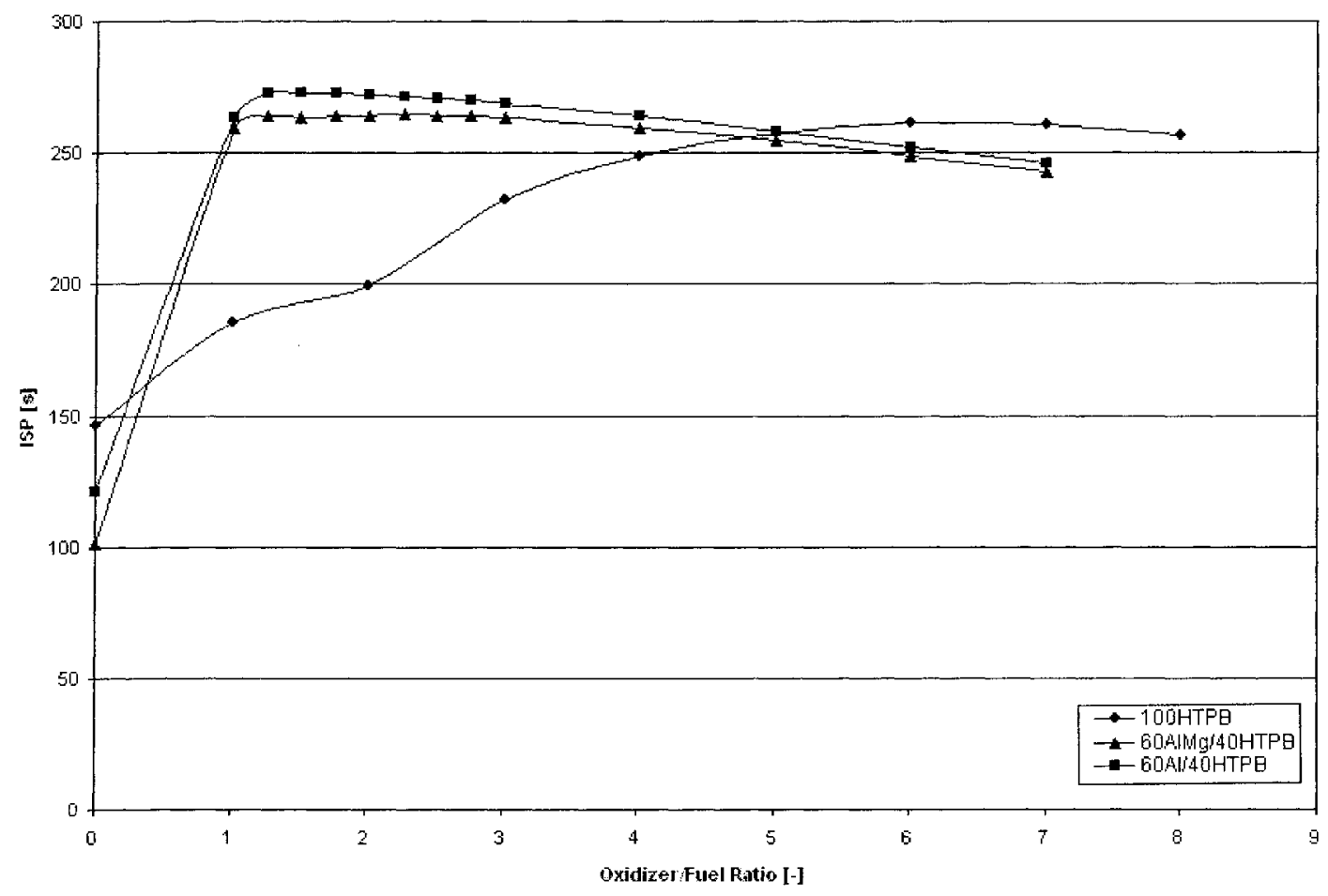

Figure 2.2: Theoretical performance of HyperG propellants: ISP 


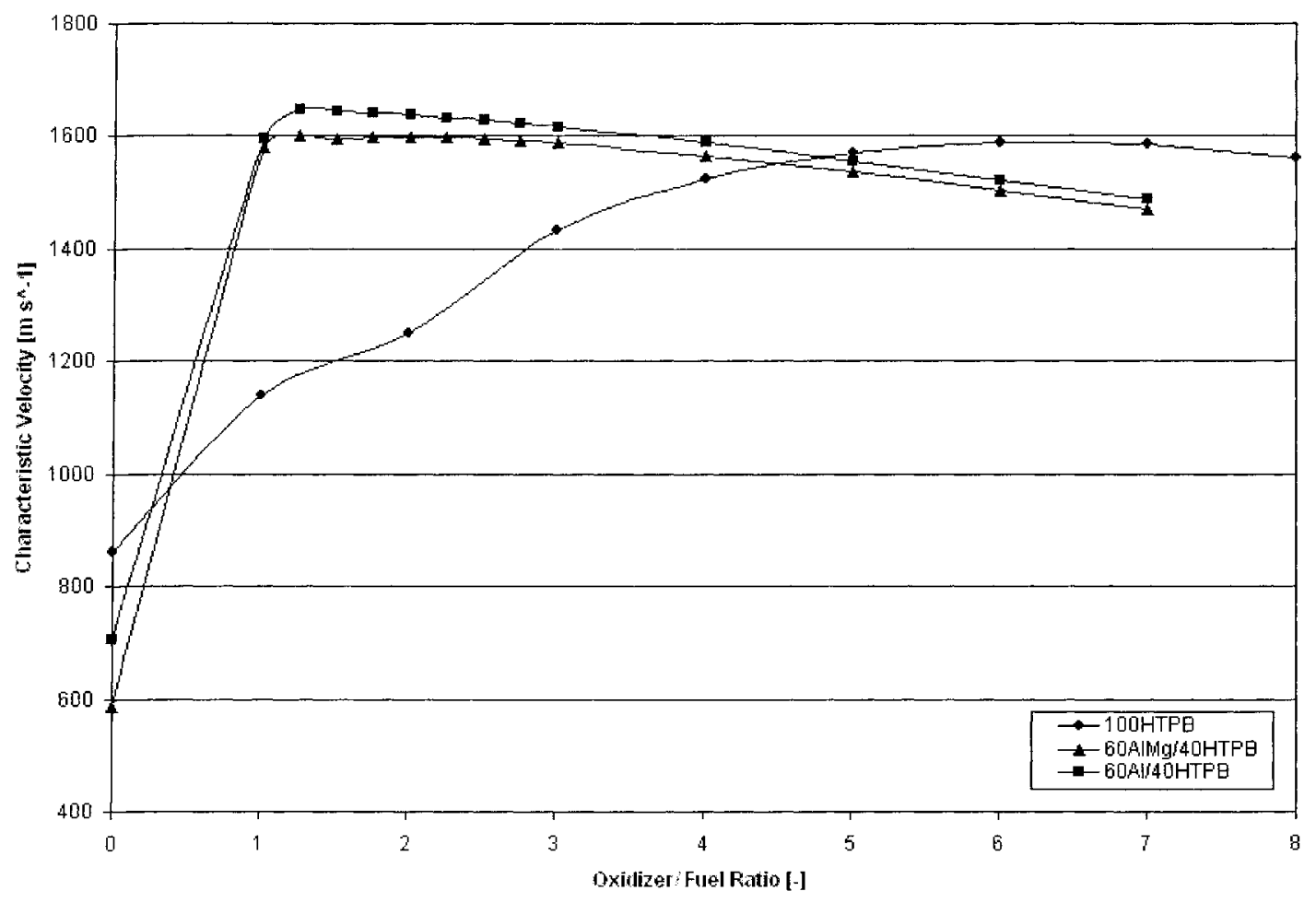

Figure 2.3: Theoretical performance of HyperG propellants: c* 


\subsection{Propellant}

Decomposed $90 \% \mathrm{HP}$ and HTPB are hypergolic at the decomposition temperature, meaning that they ignite spontaneously on contact. The exhaust of decomposed HP is nontoxic. The exhaust of the propellant combinations used in the HyperG experiments was also nontoxic.

In the HTPB/90\%HP tests, the HTPB was the only fuel. In the $60 \% \mathrm{Al} / 40 \% \mathrm{HTPB} / 90 \% \mathrm{HP}$ and $60 \% \mathrm{AlMg} / 40 \% \mathrm{HTPB} / 90 \% \mathrm{HP}$ tests, the HTPB was a secondary fuel but also acted as the binder material for the metal particles. When the HTPB was ablated during the burn, the metal particles were carried into the boundary layer by the gaseous products of the HTPB decomposition, where they began to combust.

\subsubsection{Hydrogen peroxide specifications}

$90 \%$ by weight, concentrated, unstabilized hydrogen peroxide was procured from FMC Corporation, Bayport, Texas. Stabilizers typically used in HP are stannate and phosphate compounds [4], these compounds would have reduced the catalytic activity of the of the material used in the catalyst pack. The adiabatic flame temperature was quoted as $740^{\circ} \mathrm{C}[26]$. The composition of $90 \% \mathrm{HP}$ after the decomposition reaction given in Equation 2.1 was $42.7 \% \mathrm{O}_{2}, 57.3 \% \mathrm{H}_{2} \mathrm{O}$. Note that the $\mathrm{HP}$ was kept in storage at $\mathrm{CTI}$ at $70-80^{\circ} \mathrm{F}$ for approximately a year prior to its use, and a typical 
decomposition rate of HP stored under these conditions is slightly greater than $1 \%$ a year $[27]$.

$$
\mathrm{H}_{2} \mathrm{O}_{2}(l) \rightarrow \frac{1}{2} \mathrm{O}_{2}(g)+H_{2} \mathrm{O}(g)+\triangle H_{\text {decomp }}
$$

\subsubsection{Fuel formulations}

The fuel compositions and particle sizes of the aluminum and aluminum/magnesium additives that were used are summarized in Table 2.1. The HTPB formulation used in these tests was Poly bd R45HT. The fuel additives and their uses are summarized in Table 2.2. Not every additive was used in each fuel. Carbon black was added to the non-metallized HTPB fuel to render it opaque, reducing the amount of radiation transmitted through the grain during curing. In general, the fuel was mixed under a vacuum and cured for at least two weeks prior to use at the CTI facilities in Gormley, Ontario.

Table 2.1: Metallic particle characteristics.

\begin{tabular}{|c|c|c|}
\hline Fuel composition & $\begin{array}{l}60 \% \mathrm{Al} / 40 \% \mathrm{HTPB} \text { by } \\
\text { weight }\end{array}$ & $\begin{array}{l}60 \% \mathrm{AlMg} / 40 \% \mathrm{HTPB} \\
\text { by weight }\end{array}$ \\
\hline Metal composition & aluminum & $\begin{array}{l}50 \% \text { aluminum } / 50 \% \\
\text { magnesium alloy by } \\
\text { weight }\end{array}$ \\
\hline Particle size/shape & 1-3 $\mu m$ spherical & $\begin{array}{l}200 \text { mesh (approx. } 127 \\
\mu m \text { ) spherical }\end{array}$ \\
\hline
\end{tabular}


Table 2.2: Fuel additives and their uses.

\begin{tabular}{|l|l|l|}
\hline Item & Chemical Name & Use \\
\hline \hline Poly bd R45HT & HTPB & binder resin \\
Antifoam & - & - \\
Carbon black & - & render fuel grain opaque \\
Cabosil & silicon dioxide & resin thickener \\
FEAA solution & ferric acetyl acetonate & curative catalyst \\
DBTDL solution & dibutyltin dilaurate & curative catalyst \\
DOA & dioctyl adipate & plasticizer \\
Desmodur W & dicyclohexylmethane diisocyanate & curing agent \\
\hline
\end{tabular}

\subsection{Test hardware}

A large portion of the thrust bench hardware and rocket motor components were either assembled at CTI prior to the start of the work described herein, or were modified versions of existing hobby motor components from the CTI Pro75 motor line. As such, a mainly qualitative overview of the experimental hardware will be given in this section.

\subsubsection{Catalyst pack}

High test hydrogen peroxide decomposes according to Equation 2.1 in the presence of heat and impurities and a catalyst pack is generally used to accomplish this in HP gas generator and mono-propellant systems. The catalyst pack used for HyperG was designed based on a literature review of previous experiments that had been performed to characterize the performance of various pack configurations. The best 
success had been obtained with various configurations of silver screen catalysts, and this was the configuration chosen.

The catalyst pack was $60 \mathrm{~mm}$ in diameter and composed of silver-plated nickel wire mesh screens that were treated with samarium nitrate to improve durability and efficiency. The screens were silver-plated with a high current to achieve a rough surface finish in order to increase surface area and flow turbulence and therefore improve the HP decomposition efficiency. The screens were arranged so as to optimize the oxidizer distribution and flow, from coarse to fine mesh starting at the injector plate. A sintered stainless steel material was used as the injector plate. The screens were contained in a $316 \mathrm{~L}$ stainless steel assembly with an aluminum bulkhead separated by a linen phenolic heat shield and supported downstream of the injector by a wagon wheel type stainless steel support. $316 \mathrm{~L}$ stainless was chosen due to its compatibility with high test hydrogen peroxide.

Images of the catalyst pack components are shown in Figures 2.4, 2.5, and 2.6. An image of $45 \% \mathrm{HP}$ decomposing on a finished screen is shown in Figure 2.7 $[2][28]$.

\subsubsection{Rocket motor components}

The HyperG motor hardware was a modified version of the CTI Pro75 hobby rocket motor. The Pro hobby motor line is a cartridge, re-loadable system with modular 


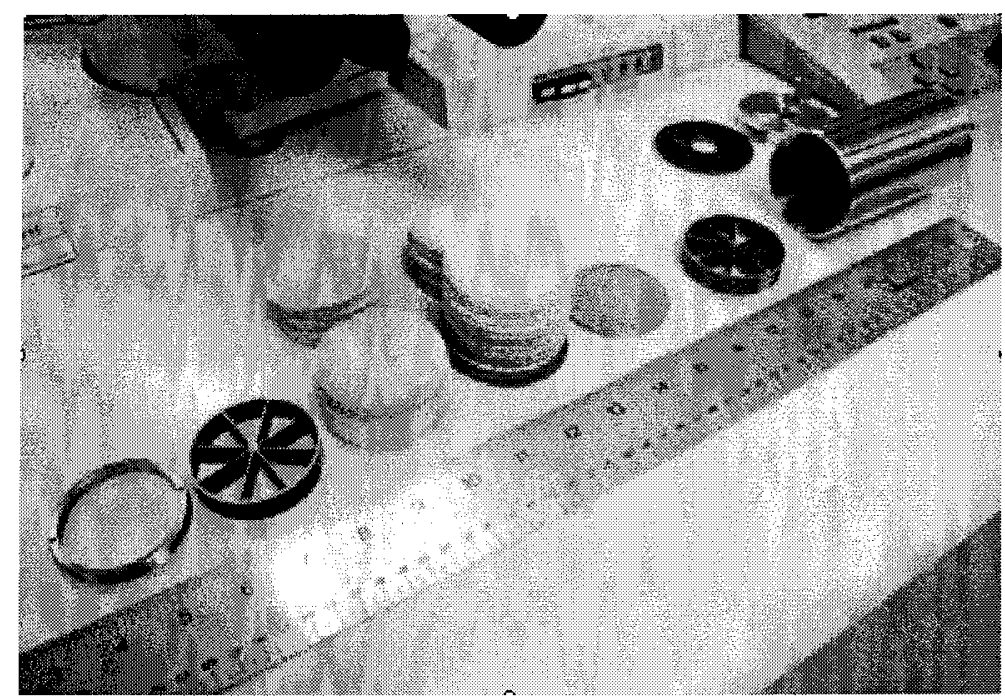

Figure 2.4: Catalyst pack and injector components.

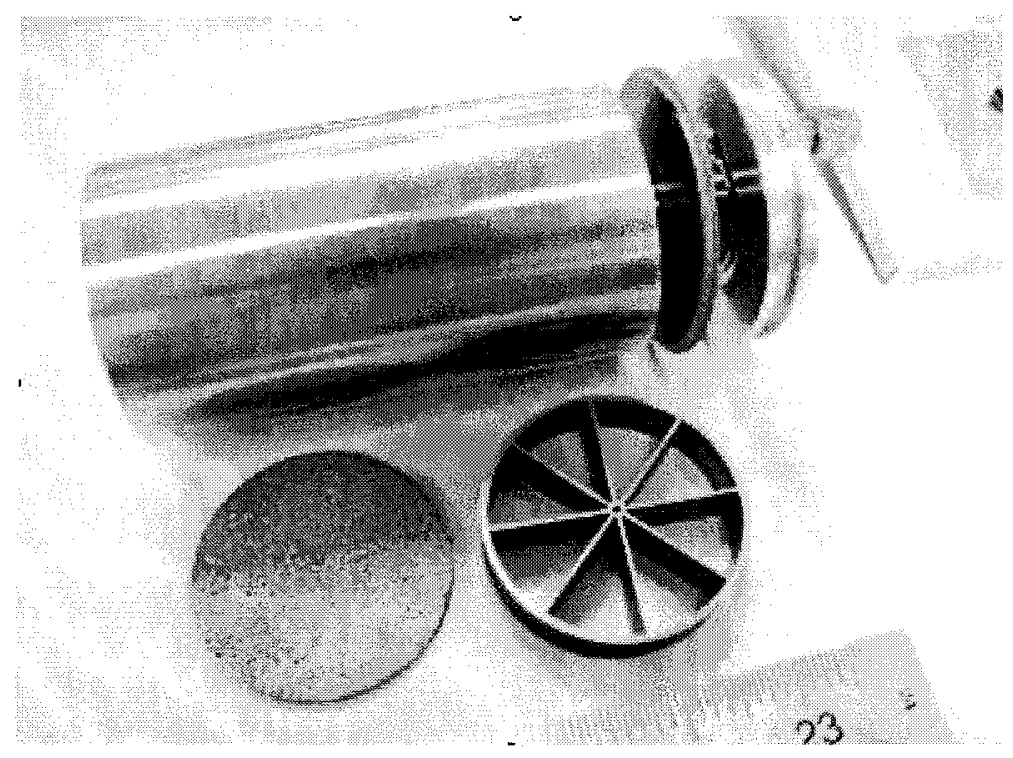

Figure 2.5: Catalyst pack and injector assembly. 


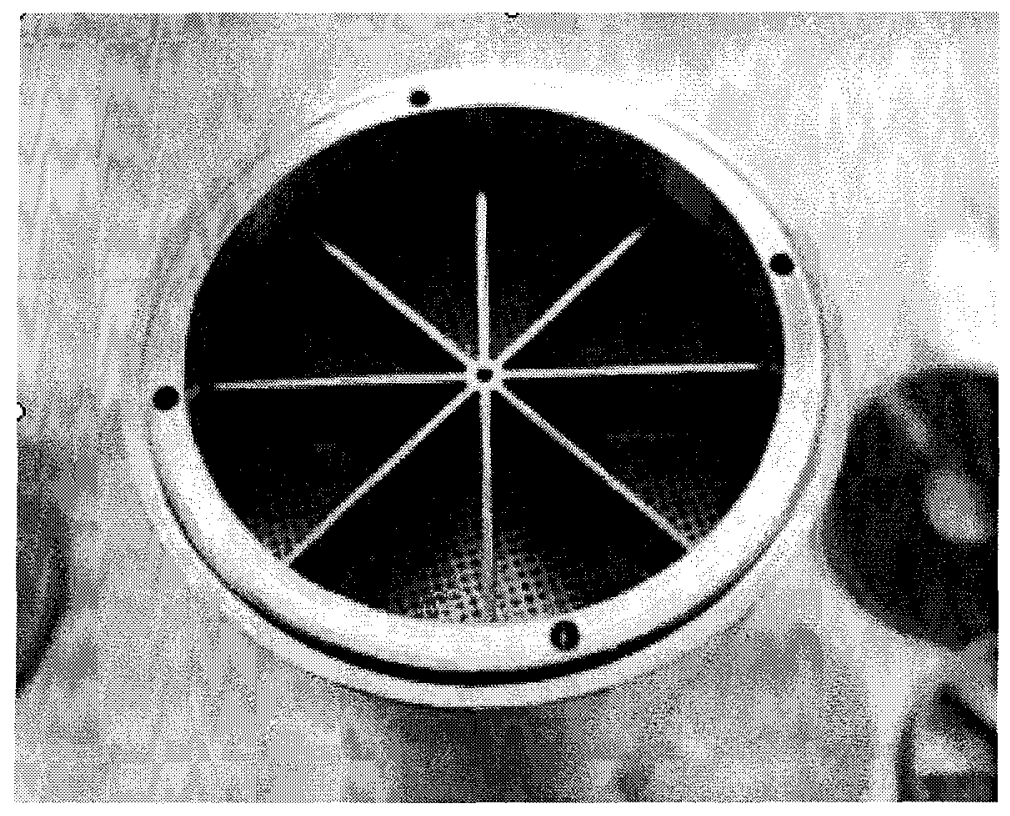

Figure 2.6: Support for catalyst screens.

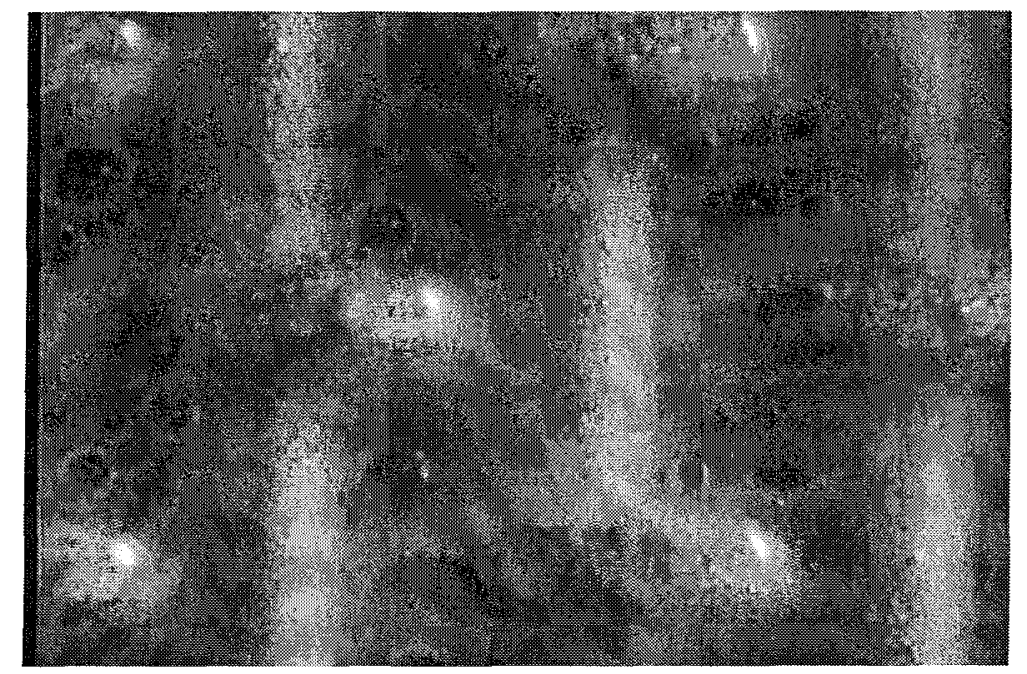

Figure 2.7: Decomposition of $45 \% \mathrm{HP}$ on catalyst screen. 
forward and aft closures. The conical nozzles were compression molded using a silica phenolic resin, and a graphite insert was then bonded into the throat. The throat and nozzle diameters were then post machined to the desired dimensions to achieve the required chamber pressure and to expand to atmospheric pressure.

Theoretically, the hot products of decomposition of the hydrogen peroxide are hypergolic with HTPB, and as such an igniter was not used to initiate combustion.

Table 2.3 summarizes the components of the HyperG motor assembly and materials of manufacture. Figure 2.8 shows the different case lengths, case liner and foward/aft closures for the Pro75 line. Figure 2.9 shows an assembly view of the motor with the catalyst pack in place [2].

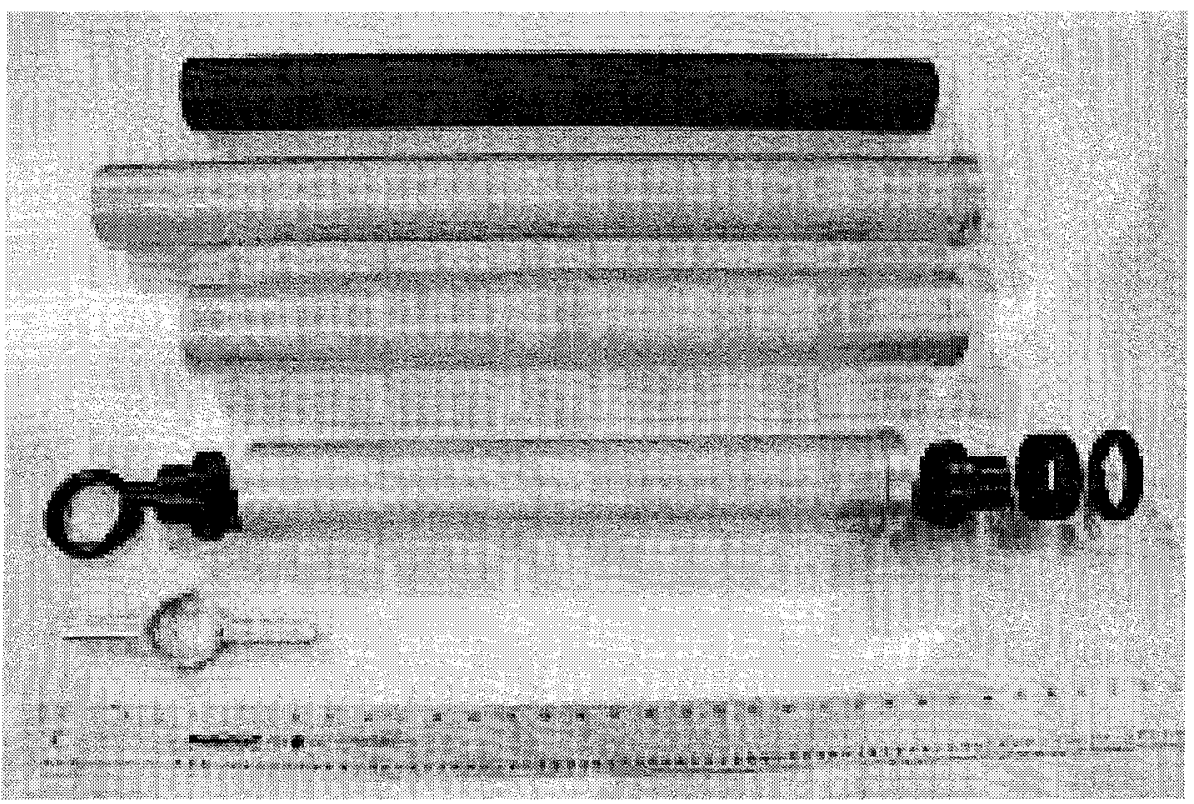

Figure 2.8: Cases, liner, and forward and aft closures for Pro75 hobby rocket line. 


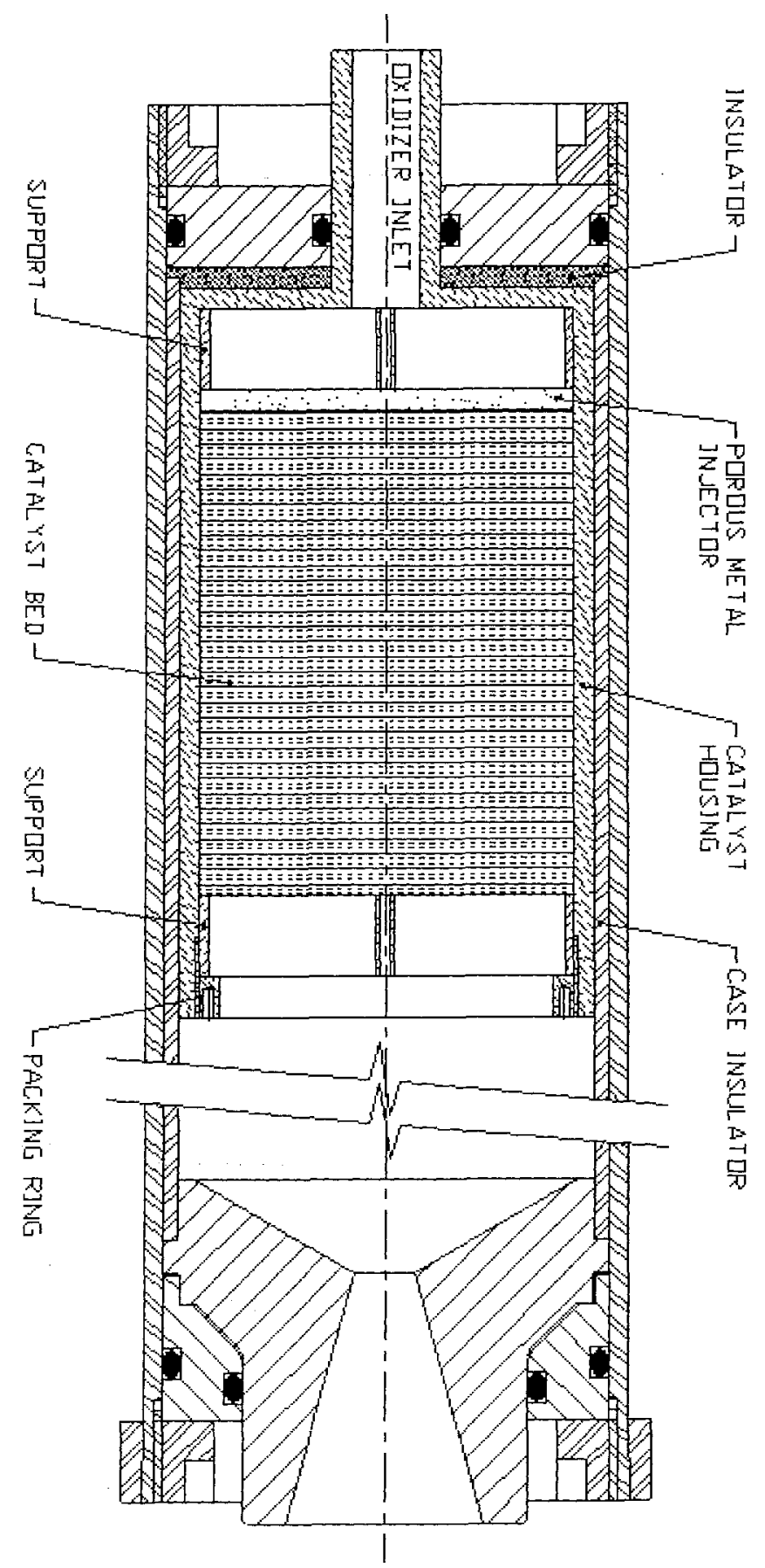

Figure 2.9: Assembly drawing of the HyperG motor. 
Table 2.3: Materials of construction for the HyperG motor and catalyst pack components.

\begin{tabular}{|l|l|}
\hline Component & Material \\
\hline \hline Catalyst Pack & \\
\hline wire mesh screens & silver plated nickel, treated with samarium nitrate \\
supports, packing ring & $316 \mathrm{~L}$ stainless steel \\
injector plate & sintered porous stainless steel \\
forward insulator & linen phenolic \\
forward bulkhead & aluminum \\
bulkhead o-rings & Buna-N \\
housing & $316 \mathrm{~L}$ stainless steel \\
\hline Other Motor Components & \\
\hline motor casing & aluminum \\
case liner & paper phenolic \\
precombustion and reaction chambers & linen phenolic \\
nozzles & molded silica phenolic, graphite insert \\
forward and aft closures & molded silica phenolic \\
retainer rings & molded silica phenolic \\
forward/aft closure o-rings & Buna-N \\
\hline
\end{tabular}

\subsubsection{Thrust bench hardware and instrumentation}

The thrust bench hardware was built entirely of Swagelok stainless steel 316L tube, fittings, and valve components. A CTI Hypertek tank was used for the oxidizer storage (Hypertek is the CTI nitrous oxide/thermoplastic hybrid motor line). Figure 2.10 shows a schematic of the test stand assembly.

The pressure transducers in this system are denoted with a "P". In the remainder of this document they are referred to by number starting from the main oxidizer tank. For example, the pressure tap downstream of the venturi is thus 
designated "P3". The temperature transducers were not installed.

The dump valve was used for emergency HP purge to a small tank in the test cell. Neither this nor the emergency water purge was required during any of the HyperG tests. The main oxidizer valve, preheat valve and dump valve were all actuated pneumatically using the compressed air supply in the compound from the test cell bunker/control room. The main oxidizer and preheat tanks were filled manually immediately prior to the start of each test, and both the manual fill valves and the vent filter valve were left open until the tank fill was complete. At that time the two fill valves were closed, and the vent valve was moved to the nitrogen fill position. A 5000 psi nitrogen cylinder with a regulator was used to pressurize the preheat and main oxidizer tanks using a three-way servo-valve. When the system was not pressurized, it was automatically venting through this valve.

The pressure relief valve was installed to allow venting of the system should the main or preheat tanks become contaminated, causing uncontrolled decomposition of the hydrogen peroxide during the time period between the tank fill and the start of the test. P1 also provided a means by which to monitor the tank pressure for decomposition. Such an event did not occur during any of the HyperG tests.

The pressure transducer at the combustion chamber (P4) was fed from the chamber by means of a small channel machined along the side of the catalyst housing, and through the forward insulator to a fitting in the forward bulkhead. These 
features are visible in Figures 2.4 and 2.5.

The cavitating venturi located upstream of P3 in Figure 2.10 initially was the $1.0 \mathrm{~mm}$ diameter throat venturi, and was replaced with the $1.5 \mathrm{~mm}$ diameter throat venturi for Tests $7,8,11,12$. Each venturi was passivated with a nitric acid solution prior to installation in the assembly to remove any surface contaminants from the machining process. Once the test assembly was used with HP it was not flushed with water between tests to avoid risk of contamination of the internal component surfaces.

Figure 2.11 is an image of the test cell before a test. Note the location of the four pressure transducers in the image; the amplifier for these sat to the left of the test stand and shielded BNC cables were used to deliver the signal to the control room. Each transducer had a slightly better than $2 \mathrm{mV} / \mathrm{psi}$ sensitivity. The thrust was measured using a 300 lbf Omega instruments load cell; the signal was amplified by the unit on the right cell wall and was delivered to the control room underground.

\subsubsection{Venturi calibration}

A cavitating venturi was used in the HyperG experiments as a mechanically simple means by which to provide instantaneous oxidizer mass flow measurements. The cavitating venturi decoupled any chamber pressure fluctuations from the mass flow 


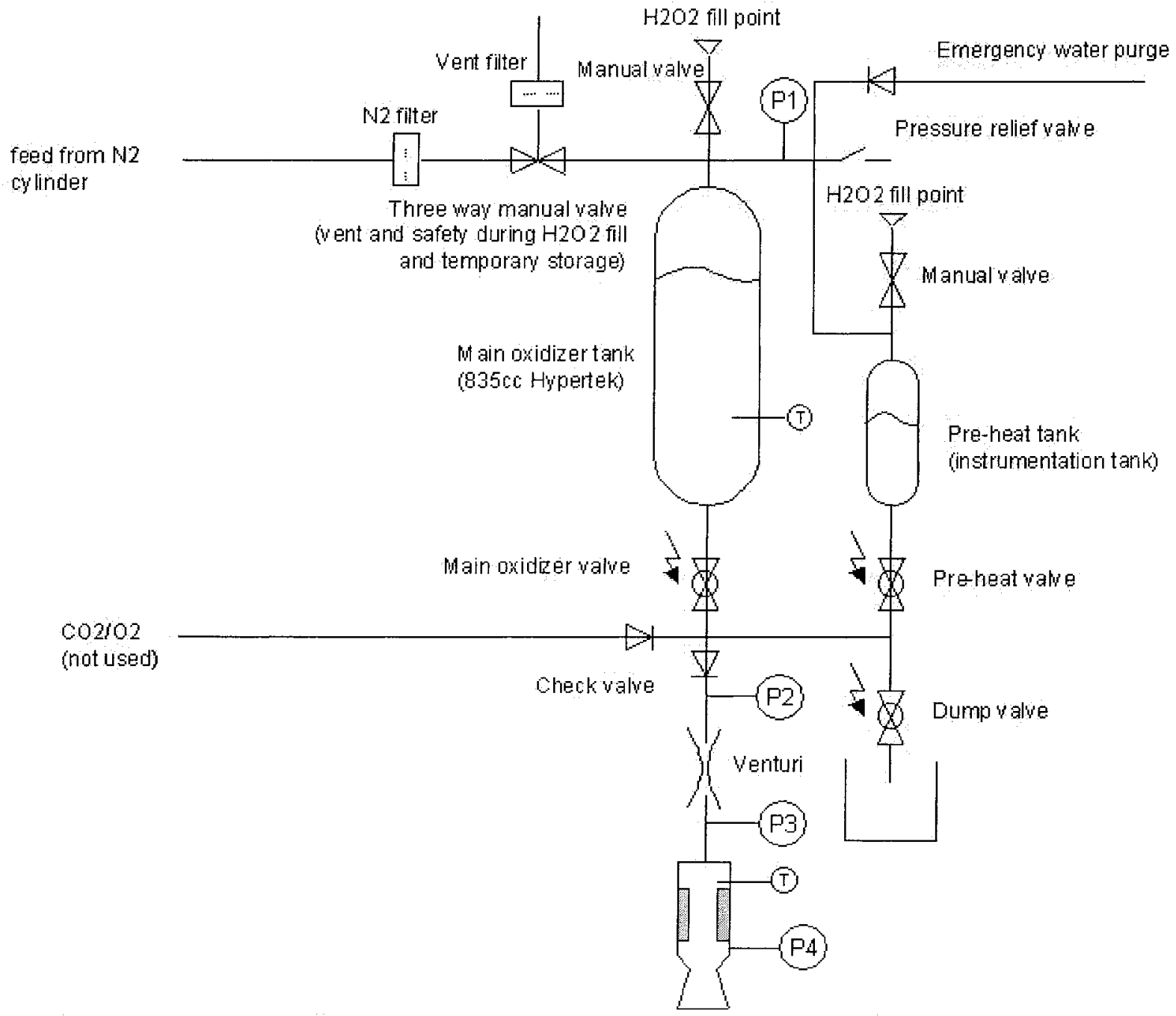

Figure 2.10: Schematic of the HyperG thrust bench assembly. 


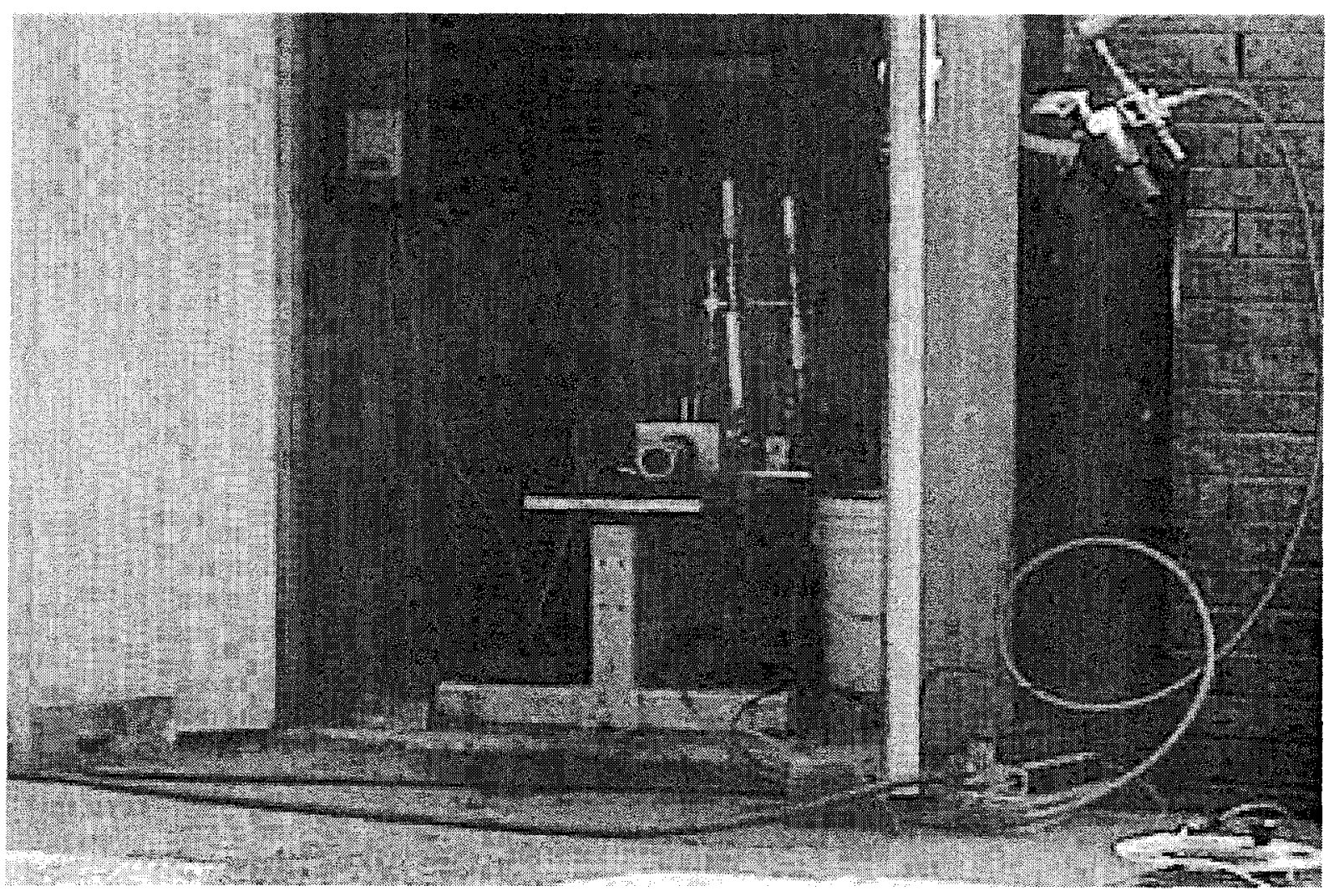

Figure 2.11: Photograph of the HyperG assembly in the test cell. 
rate of the oxidizer and ensured that it remained constant throughout the duration of each test. A venturi with a $1.0 \mathrm{~mm}$ diameter throat was used for all tests except Tests $7,8,11,12$. In those tests a venturi with a $1.5 \mathrm{~mm}$ diameter throat was used in order to increase the chamber pressure by increasing the oxidizer mass flow, allowing the nozzle throat diameter to be kept at a safe size.

Assuming a pressure drop large enough to cause cavitation at the throat, the mass flow through a cavitating venturi is calculated using Equation 2.2. The loss coefficient in Equation 2.2 must be quantified by a calibration of the device.

$$
\dot{m}_{o x}=\sqrt{2 \rho_{H P} \frac{\left((P 2+P a t m)-P_{v, H P}\right)}{\frac{1}{C_{c}^{2} A_{\text {throat }}^{2}}-\frac{1}{A_{\text {pipe }}^{2}}}}
$$

$P_{v, H P}=358.63 \mathrm{~Pa}$ is the vapour pressure of the $\mathrm{HP}$ at $25^{\circ} \mathrm{C}, \mathrm{P} 2$ is the pressure immediately upstream of the venturi, $A_{p i p e}$ is the pipe area immediately upstream of the venturi, $A_{t h r o a t}$ is the venturi throat area. The venturi was machined in such a way that the throat had a sharp edge rather than a smooth radius. As such, $\mathrm{C}_{c}$ is the contraction coefficient representing the reduction in throat area due to the formation of a vena contracta, as well as other losses due to a departure from ideal frictionless flow. $\mathrm{C}_{c}$ was quantified using the calibration process described below.

Distilled water was pressurized and then passed through the system, collected and the mass measured. Equation 2.2 was then numerically integrated using an 
iterative procedure to determine the correct value of $\mathrm{C}_{c}$ such that the integrated mass equalled that which was measured. The calibration coefficient may have been slightly different for hydrogen peroxide than for water due to the slight difference in molecular viscosity of the two fluids, however this could not be safely avoided as it was not safe to calibrate the venturi with hydrogen peroxide, due to the possibility of skin exposure during the process. Also, it was not possible to accurately account for the small amount of liquid that likely remained trapped in the dump lines of the HyperG test hardware downstream of the venturi during the calibration. Thus, a small error was introduced into the mass flow measurement due to these effects that was not accounted for in the error analysis presented in Table 2.5.

Calibration of the $1.5 \mathrm{~mm}$ venturi using the same method failed due to the high pressures required to achieve a mass flow rate that caused cavitation. The high pressures prevented the water from being collected accurately at the outlet of the venturi. The data reduction for Tests $7,8,11,12$ was performed using the measured value for the mass of hydrogen peroxide that was taken before each test rather than integrating the oxidizer flow rate calculated at each time step. Note that the venturi was cavitating so that a constant oxidizer mass flow rate over the duration of the test was assumed. 


\subsubsection{Data acquisition}

Data collection was accomplished using Cesaroni in-house data acquisition (DAQ) software, DATACAD, and a Measurement Computing PCI-DAS6013 card. The card had sixteen 16 bit channels, each sampled at 1000 samples/second, and did not filter the sampled data before writing to disk. Five channels were used for each test, four for the pressure transducers and one for the load cell. The DATACAD software allowed user programmable channel calibration coefficients enabling real-time pressure and thrust measurements to be displayed on screen prior to the initiation of data collection. The DAQ PC was located inside the control room.

\subsection{Test procedure}

Before the test program was initiated, HP was flushed through the catalyst pack in successive concentrations of approximately $60 \%, 70 \%, 85 \%$, and $90 \%$, in order to safely ensure that the pack would perform as expected for the tests. A significant change in the colour of the exhaust from the catalyst pack was noted. The exhaust at $60 \% \mathrm{HP}$ appeared almost entirely white, and become progressively more clear with each increase of concentration. At $85 \%$ concentration, white exhaust was only visible during the initial second of the flush. Flushing $90 \% \mathrm{HP}$ through the catalyst pack resulted in a completely clear exhaust throughout the duration of the flush. The clear nature of the exhaust signified complete vapourization of the water in the 
HP decomposition products. The motor was fired in a mono-propellant configuration initially in an effort to quantify the efficiency of the catalyst pack. Detailed results of that test are not included in this document, as the motor liner began to combust approximately seven seconds into the burn making a reliable determination of catalyst pack efficiency impossible.

A generic description of the typical test procedure follows.

1. Fuel grain was weighed, motor assembled, weighed and attached to thrust bench.

2. A peristaltic pump was used to fill the HP transfer bottle. Main and preheat tanks were filled with HP. Masses of HP transfer bottle were taken prior to main fill, prior to preheat tank fill and after preheat tank fill.

3. DAQ channels were zeroed, tanks were pressurized, a small amount of HP from preheat tank was flushed through catalyst pack 2-3 times to preheat heat catalyst, tanks depressurized.

4. DAQ was started, tanks were pressurized, valve to main oxidizer tank was opened.

5. After firing was complete, nitrogen was flushed through both preheat tank and main tank feed lines, tanks were depressurized. 
6. Motor, grain, nozzle, precombustion chamber and reaction chamber were weighed and measured.

\subsection{Data reduction}

The reading from pressure tap $\mathrm{P} 4$ (chamber pressure) lagged behind that from P3 during the initial seconds of the tests, and the channel that ran from the combustion chamber to the port in the bulkhead became blocked by accumulated soot from the motor. To avoid this difficulty, the pressure reading from transducer P3 was used as the chamber pressure in the data analysis. Figure 2.12 from Test 1 shows an example of the lag of the $\mathrm{P} 4$ transducer reading behind the $\mathrm{P} 3$ reading. When this figure is scaled to show a fraction of the test duration in Figure 2.13, the very small difference between the $\mathrm{P} 3$ and $\mathrm{P} 4$ readings is clear; this difference is a measure of the pressure drop across the catalyst pack. This figure indicates that the overestimation of the chamber pressure due to using the P3 transducer reading was no more than 15 psi. This value was included in the error analysis in Section 2.6 .2 as the worst case estimated error on the measured $\mathrm{P}_{c}$ value.

Because the venturi with the $1.5 \mathrm{~mm}$ throat diameter that was used in the high pressure tests (Tests $7,8,11,12$ ) was not calibrated, the mass of HP measured during the tank fill procedure was used in the data analysis as opposed to the following integral. 


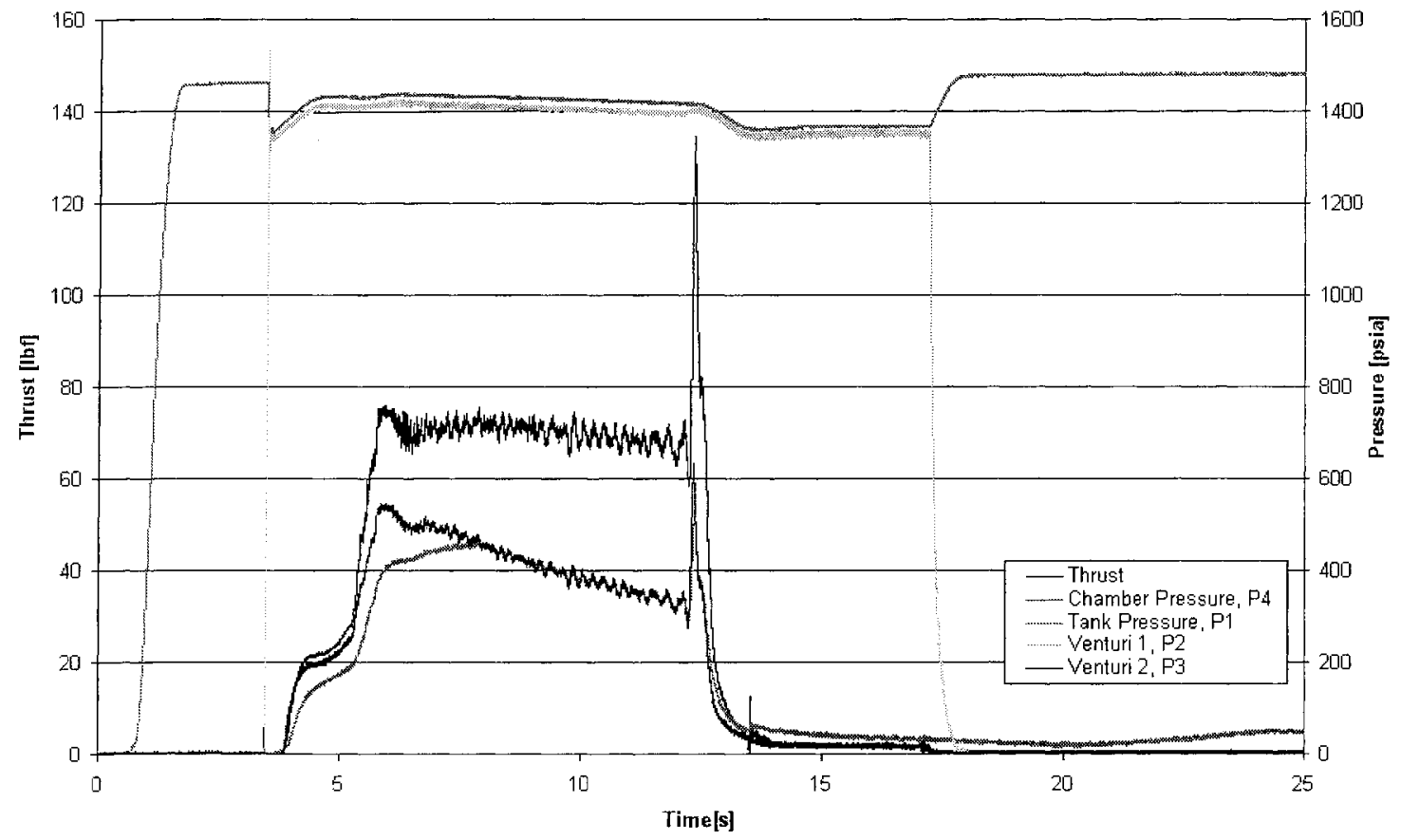

Figure 2.12: Data for Test 1 showing the lag of the P4 transducer at the start of the test. 


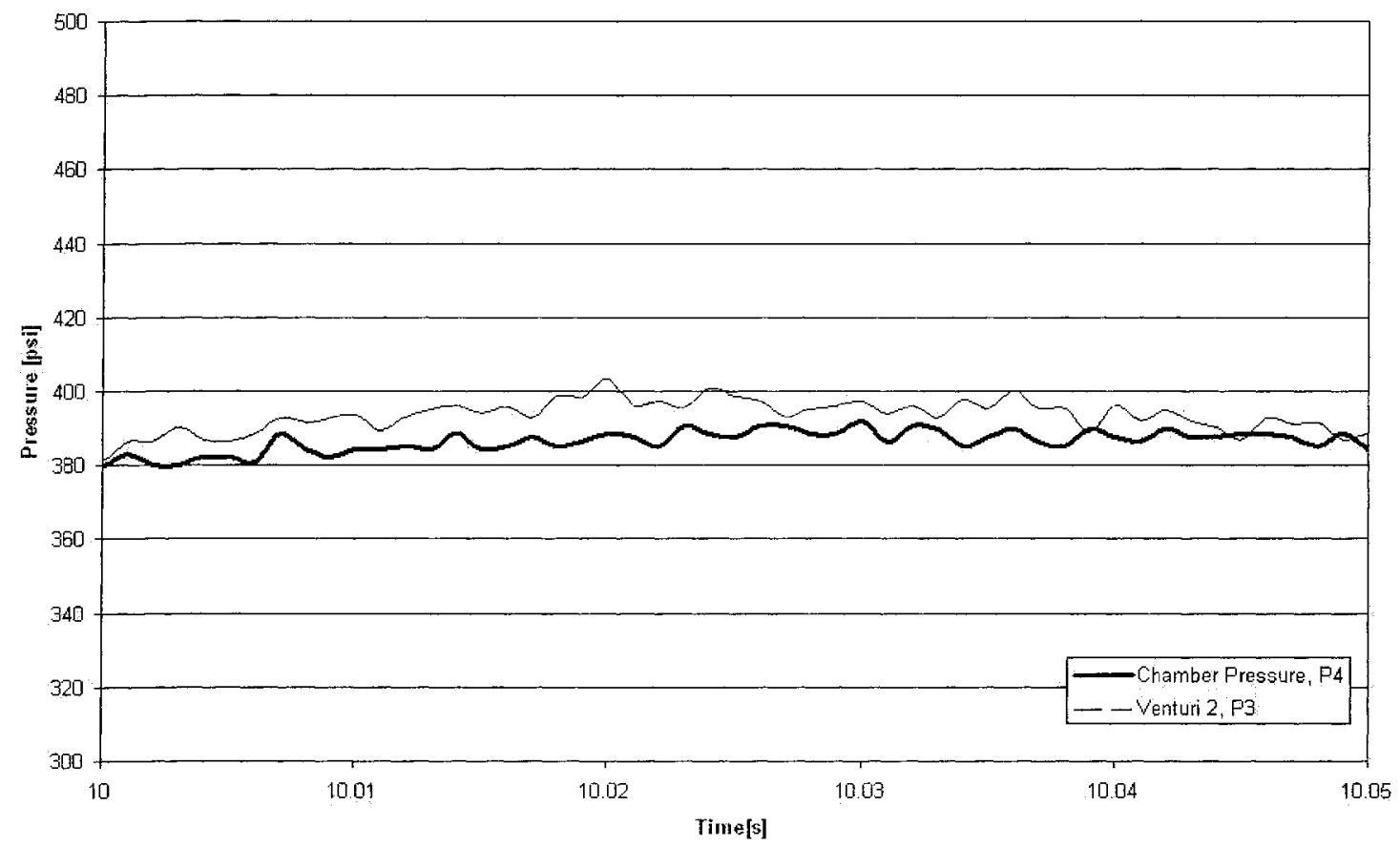

Figure 2.13: $0.05 \mathrm{~s}$ of data for Test 1 showing the small difference between the P3 and $\mathrm{P} 4$ transducer readings. 


$$
M_{o x}=\int_{t i}^{t f} \dot{m}_{o x} d t
$$

\subsubsection{Time averaged analysis}

The data was exported to a capture file in raw voltage format to allow calibration of the channels and bias removal using the Excel software package. All subsequent data analysis was accomplished using Excel and the Visual Basic for Applications programming language. Time averaged data reduction was performed by calculating parameter averages over the action time of the motor, consistent with the analysis presented in [3]. Integrals were calculated numerically using trapezoidal integration. The theoretical specific impulse and characteristic velocity were calculated using the program described in [25]. The motor start time was taken as the first movement on the chamber pressure trace that was greater than the rms fluctuation amplitudes, and the stop time was determined using the "tan-bisector" method as described in [1] and consistent with the method used in [3].

The temporally and spatially averaged regression rate, assuming that the grain regressed cylindrically, was calculated using the mass of fuel consumed during the test.

$$
\overline{\dot{r}}=\frac{1}{t_{a}}\left[\left[\frac{\Delta M_{f}}{\pi \rho_{f} L_{\text {grain }}}+\left(\frac{\text { Dport }_{i}}{2}\right)^{2}\right]^{\frac{1}{2}}-\frac{\text { Dport }_{i}}{2}\right]
$$


The mass flow rates of the inert material (precombustion and reaction chambers) and fuel were temporally averaged.

$$
\begin{gathered}
\overline{\dot{m}_{\text {inert }}}=\frac{\Delta M_{\text {inert }}}{t_{a}} \\
\overline{\dot{m}_{f}}=\frac{\Delta M_{f}}{t_{a}}
\end{gathered}
$$

The temporally averaged oxidizer mass flux and total mass flux were calculated using an average port area, integral of the instantaneous oxidizer mass flow rate over the action time, and the consumed mass of fuel. The subscripts 1 and 2 in Equation 2.7 refer to measurements taken at the start and end of the grain respectively.

$$
\begin{gathered}
\overline{\text { Aport }}=\frac{\pi}{64}\left[\left(\text { Dport }_{f}+\text { Dport }_{i}\right)_{1}+\left(\text { Dport }_{f}+\text { Dport }_{i}\right)_{2}\right] \\
\overline{G_{o x}}=\frac{\int_{t i}^{t f} \dot{m}_{o x} d t}{\overline{A p o r t} \cdot t_{a}} \\
\overline{G_{t o t}}=\overline{G_{o x}}+\frac{\Delta M_{f}}{\overline{A p o r t} \cdot t_{a}}
\end{gathered}
$$

The temporally averaged OF ratio was calculated as follows: 


$$
\overline{O F}=\frac{\int_{t i}^{t f} \dot{m}_{o x} d t}{\Delta M_{f}}
$$

The temporally averaged chamber pressure was calculated as follows:

$$
\overline{P_{c}}=\frac{1}{t_{a}} \int_{t i}^{t f}(P 3+P a t m) d t
$$

The temporally averaged ISP is a measure of the "propellant efficiency" or the thrust per unit weight of propellant. The ISP efficiency reflects losses due to incomplete exhaust expansion, flow separation, surface roughness, two phase nozzle flow, non-ideal gas behaviour.

$$
\begin{gathered}
\overline{I S P}=\frac{\int_{t i}^{t f} F d t}{g\left(\int_{t i}^{t f} \dot{m}_{o x} d t+\Delta M_{f}+\Delta M_{\text {inert }}\right)} \\
\overline{\eta I S P}=\frac{\overline{I S P}}{I S P_{\text {theory }}}
\end{gathered}
$$

The temporally averaged characteristic velocity and characteristic velocity efficiency are a measure of the thermochemical merit of the propellant combination and the combustion efficiency obtained during the test respectively. $A_{\text {throat }}$ refers to the instantaneous throat area of the nozzle. The instantaneous nozzle throat area was calculated by assuming that the nozzle throat regressed linearly during the test. The total regressed distance of the nozzle throat was measured after each test, and 
a fraction was added to the original throat diameter at each time step in the data analysis.

$$
\begin{gathered}
\overline{c^{*}}=\frac{\int_{t i}^{t f}(P 3+\text { Patm }) A_{\text {throat }} d t}{\int_{t i}^{t f} \dot{m}_{o x} d t+\Delta M_{f}+\Delta M_{\text {inert }}} \\
\overline{\eta c^{*}}=\frac{\overline{c^{*}}}{c_{\text {theory }}^{*}}
\end{gathered}
$$

Because the amount of erosion of the nozzle throat was substantial during some of the tests, the average combustion efficiency given by Equation 2.15 proved to be erroneously high in those tests. The quality of graphite used in the nozzle throat inserts was improved after Test 5, this will be discussed further in Section 2.7.5. To obtain valid results for the affected tests, the combustion efficiency was estimated from the calculated ISP value using an ideal coefficient of thrust $\left(\mathrm{Cf}_{\text {ideal }}\right)$ which allowed a value for $\overline{\eta c^{*}}$ to be obtained without the use of $\mathrm{A}_{\text {throat }}$. The coefficient of thrust was calculated using the ratio of specific heats $(\gamma)$ for the propellant composition assuming ideal gas behaviour, obtained from PROPEP, and the ratio of nozzle exit pressure to combustion chamber pressure, $\frac{P_{e x i t}}{P_{c}}$, for each test. Because the nozzle throat erosion caused this ratio to change, $\mathrm{Cf}_{i d e a l}$ was calculated for the start and end conditions of the test and averaged to obtain a final value. The derivation of Equation 2.16 was presented in 1.2. This method of estimating the 
combustion efficiency was also used by Lips [11].

$$
\begin{gathered}
C f_{\text {ideal }}=\sqrt{\frac{2 \gamma^{2}}{\gamma-1}\left[\frac{2}{\gamma+1}\right]^{\frac{\gamma+1}{\gamma-1}}\left[1-\left(\frac{P_{\text {exit }}}{P_{c}}\right)^{\frac{\gamma-1}{\gamma}}\right]}+\left(\frac{P_{\text {exit }}}{P_{c}}-\frac{P_{\text {amb }}}{P_{c}}\right) \frac{A_{\text {exit }}}{A_{\text {throat }}} \\
\overline{\eta c_{\text {est. }}^{*}}=\frac{1}{c_{\text {theory }}^{*} C f_{\text {ideal }}} \frac{\int_{\text {ti }}^{t f} F d t}{\dot{m}_{\text {ox }} d t+\Delta M_{f}+\Delta M_{\text {inert }}}
\end{gathered}
$$

It is important to note that the characteristic velocity obtained using Equation 2.17 actually reflects both combustion efficiency and thrust conversion efficiency or nozzle losses. However, the use of the start and end values of $\frac{P_{\text {exit }}}{P_{c}}$ when calculating $\mathrm{Cf}_{\text {theory }}$ decreased the magnitude of the losses due incomplete exhaust expansion that were included in Equation 2.17.

\subsubsection{Error analysis}

The error analysis was performed following the method presented in [29]. The method involves estimating the experimental error on $\overline{c^{*}}, \overline{I S P}$, and $\overline{\dot{r}}$ by calculating the bias error for each parameter. One writes the parameter of interest in terms of the measured parameters and then derives expressions for the partial derivatives of the parameter of interest with respect to each of the measured quantities. The total error is then the root sum square of all of the errors from the measured quantities. Table 2.4 gives the parameter errors and nominal values used in the error analysis. 
The nominal values in this table were taken from Test 14, however in general the bias error will differ for every test as a function of the nominal values for that particular test. As such the calculation must be repeated to obtain a bias error specific to each test. A basic example of the method of calculation is given in Equation 2.18 for the error on the fuel mass flow rate quantity.

$$
\begin{aligned}
& \frac{\partial \overline{\dot{m}_{f}}}{\partial \Delta M_{f}}=\frac{1}{t_{a}} \\
& \frac{\partial \overline{\dot{m}_{f}}}{\partial t_{a}}=\frac{\Delta M_{f}}{t_{a}^{2}} \\
& \text { Error }_{\overline{m_{f}}}=\left[\left(\frac{\partial \overline{\dot{m}_{f}}}{\partial \Delta M_{f}} \cdot \delta \Delta M_{f}\right)^{2}+\left(\frac{\partial \overline{\dot{m}_{f}}}{\partial t_{a}} \cdot \delta t_{a}\right)^{2}\right]^{0.5}
\end{aligned}
$$

Table 2.5 gives the experimental error calculated for each parameter of interest, applicable to Test 14. The error calculated for the experiments described in $[29]$ is also included in this table for comparison.

\subsection{Results and discussion}

The data obtained for Test 10, all channels, is shown in Figure 2.14. The regions labeled on this figure were common to each test. This test also exhibited some characteristics that occurred in the majority of the other tests and is thus used for discussion purposes in the sections that follow. 
Table 2.4: Parameter uncertainty values used in error analysis.

\begin{tabular}{|c|c|c|c|}
\hline Parameter & Nominal Value & $\pm \delta$ & Basis \\
\hline $\mathrm{P}_{c}$ & $535 \mathrm{psi}$ & $15 \mathrm{psi}$ & $\begin{array}{l}\text { estimated error due to use of P3 } \\
\text { transducer }\end{array}$ \\
\hline $\mathrm{P} 2$ & 1300 psi & 5 psi & $\begin{array}{l}\text { estimated rms fluctuation ob- } \\
\text { served }\end{array}$ \\
\hline$t_{a}$ & $8.08 \mathrm{sec}$ & $0.12 \mathrm{sec}$ & from $[29]$ \\
\hline $\mathrm{F}$ & $70 \mathrm{lbf}$ & $0.32 \mathrm{lbf}$ & $\begin{array}{l}\text { estimated rms fluctuation ob- } \\
\text { served }\end{array}$ \\
\hline$M_{f}$ initial & $1.0 \mathrm{~kg}$ & $0.001 \mathrm{~kg}$ & $1 \mathrm{gm}$ scale resolution \\
\hline$\Delta M_{f}$ & $0.2 \mathrm{~kg}$ & $0.002 \mathrm{~kg}$ & $2 \times 1 \mathrm{gm}$ scale resolution \\
\hline Dport $_{i}$ & $25.4 \mathrm{~mm}$ & $0.5 \mathrm{~mm}$ & estimated deviation of mold OD \\
\hline $\mathrm{OD}$ of grain & $64.5 \mathrm{~mm}$ & $0.5 \mathrm{~mm}$ & estimated deviation of mold ID \\
\hline grain length & $391.5 \mathrm{~mm}$ & $1.0 \mathrm{~mm}$ & estimated deviation cut length \\
\hline venturi throat diameter & $1.0 \mathrm{~mm}$ & $0.0127 \mathrm{~mm}$ & machining precision \\
\hline nozzle throat diameter & $8.0 \mathrm{~mm}$ & $0.0127 \mathrm{~mm}$ & machining precision \\
\hline $\mathrm{Cc}$ & 0.97 & 0.018 & $\begin{array}{l}\text { standard deviation from calibra- } \\
\text { tion }\end{array}$ \\
\hline$\rho_{f}$ & $907.1 \frac{\mathrm{kg}}{\mathrm{m}^{3}}$ & - & error was calculated ${ }^{a}$ \\
\hline$\rho_{H P}$ & $1387 \frac{\mathrm{kg}}{\mathrm{m}^{3}}$ & - & error assumed negligible \\
\hline$P_{\text {vapour }, H P}$ & $359 \mathrm{~Pa}$ & - & error assumed negligible \\
\hline$\overline{\dot{m}_{t o t}}$ & $0.140 \frac{\mathrm{kg}}{\mathrm{s}}$ & - & error was calculated \\
\hline & $0.669 \frac{\mathrm{mm}}{\mathrm{s}}$ & - & error was calculated \\
\hline$c_{\text {theory }}^{*}$ & $1576 \frac{\mathrm{m}}{\mathrm{s}}$ & - & theoretical calculation \\
\hline$I S P_{t h}$ & $243.5 \mathrm{sec}$ & - & theoretical calculation \\
\hline
\end{tabular}

"This phrase indicates that the parameter error was calculated using the procedure described as it was a function of other measured quantities.

Table 2.5: Experimental errors for Test 14.

\begin{tabular}{|l|l|l|}
\hline Parameter & HyperG Error & Error from Ref. [29] \\
\hline \hline characteristic velocity $\left(\overline{c^{*}}\right)$ & $53.3 \frac{m}{\mathrm{~s}}, 3.4 \%$ of nominal & $2.7 \%$ of nominal \\
$\overline{I S P}$ & $6.6 \mathrm{sec}, 2.7 \%$ of nominal & not given \\
regression rate $(\bar{r})$ & $0.015 \frac{\mathrm{mm}}{\mathrm{s}}, 2.3 \%$ of nominal & $1.9 \%$ of nominal \\
\hline
\end{tabular}


Table 2.15 is a summary of the quantitative results of each test. Specific aspects of these results are discussed in the following sections.

\subsubsection{Ignition transient}

The ignition transient observed in Test 10 (Figure 2.14) of approximately 1 second occurred in all of the tests except Test 8 . This phenomenon was primarily an indication of the residence time required to bring the fuel to ignition temperature. The short time delay of approximately 10-15 seconds between the end of the preheat and the start of the main burn, may also have allowed the catalyst pack to cool slightly; the pack would then require a short time to return to full decomposition efficiency.

The nominal one second ignition transient observed in Tests 1-5 was not eliminated in Tests 6-15 with the use of an initiator and small quantities of solid propellant bonded to the interior of the fuel grain. It was likely that there was an insufficient amount of propellant to sustain combustion until the main oxidizer tank was fired, after the preheat was completed and the DAQ was initiated.

\subsubsection{Pressure and thrust anomalies}

The gradual decrease in chamber pressure during the motor burn shown in Figure 2.14 was caused by erosion of the nozzle throat and occurred during all tests.

The rise in chamber pressure and thrust beginning at approximately 10.2 


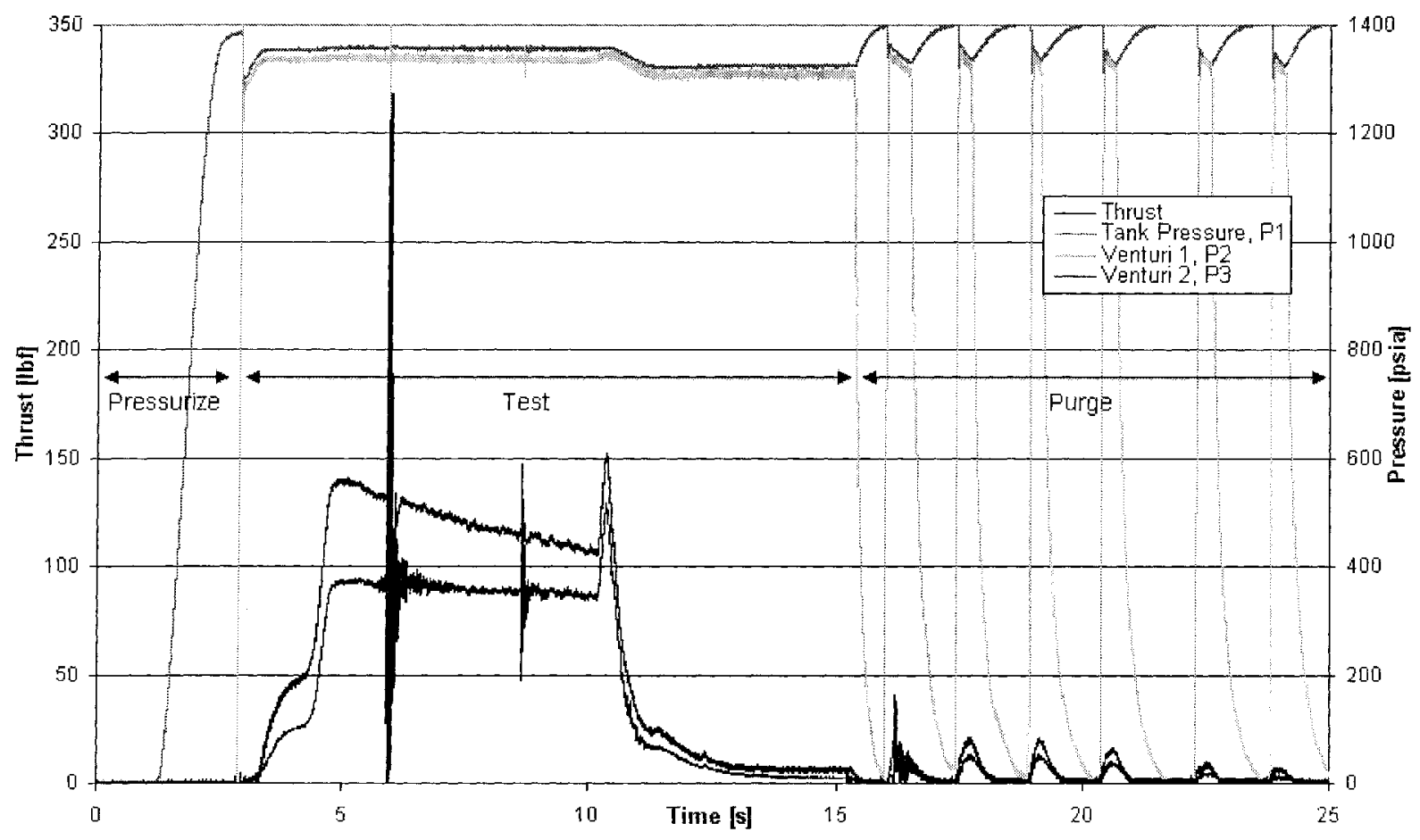

Figure 2.14: Data from all channels, Test 10. 


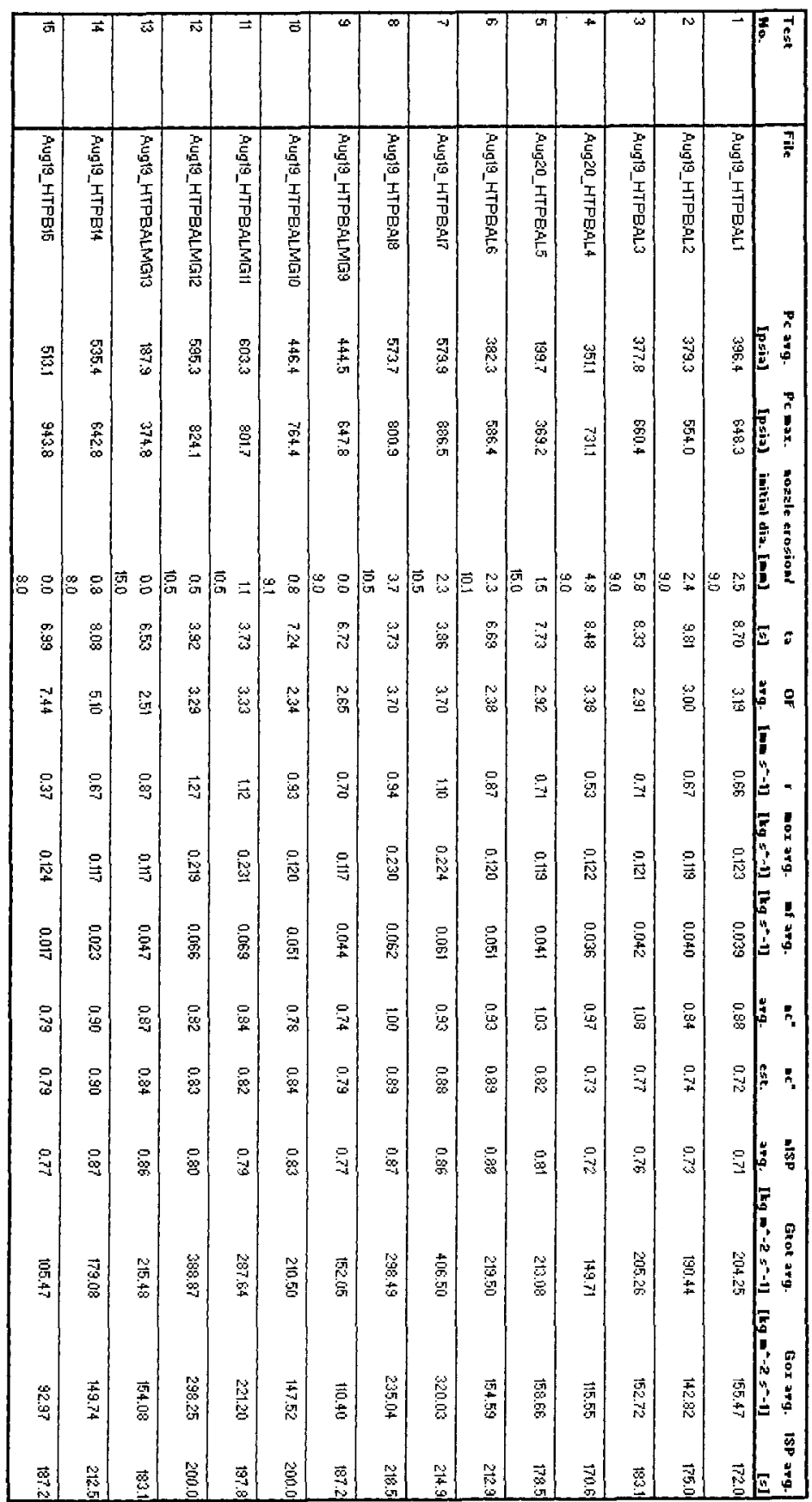

Figure 2.15: Experimental results for Tests 1-15. 
seconds into the motor action time shown in Figure 2.14 occurred during every test. This was due to high pressure nitrogen purging the motor when the main oxidizer tank had emptied, flushing out a volume of hydrogen peroxide that had accumulated in the catalyst pack.

Test 10 showed anomalous spikes in the P2, P3, and thrust measurements at approximately 5.9 seconds and 8.6 seconds. This was likely due to partial blockage of the nozzle by slag. The upstream venturi measured the anomaly because the temporarily higher chamber pressure would have caused the venturi to de-cavitate. Similar anomalies occurred in Tests 4, 11, 13, 15 .

Test 6 showed a similar spike, however the pressure anomaly led the thrust anomaly by 6 msec. and there was not a corresponding anomaly in the P2 pressure data. A possible explanation was transient combustion instability, as blockage of the nozzle by fuel slivers was unlikely with the single cylindrical port geometry of the HyperG fuel grains.

\subsubsection{Qualitative observations}

\section{$60 \% \mathrm{Al} / 40 \%$ HTPB Fuel}

While the tests run at lower chamber pressures (Tests 1-6) all exhibited solidified fuel/slag agglomeration on the reaction chamber and nozzle inlet face, this effect was greatly reduced during the higher pressure tests (Tests 7,8). A coating of fine 
aluminum oxide powder remained on the fuel grain surfaces from all tests after firing. Figure 2.16 shows slag/unreacted fuel agglomeration on the nozzle from Test 1. Agglomeration of unreacted fuel was most prevalent during Tests 1 and 2 as reaction chambers were not employed. Tests $1-5$ all exhibited visible pieces of ejected matter in the exhaust, again the effect was most prominent in Tests 1 and 2. Figure 2.17 shows the aluminum oxide powder on a spent fuel grain.

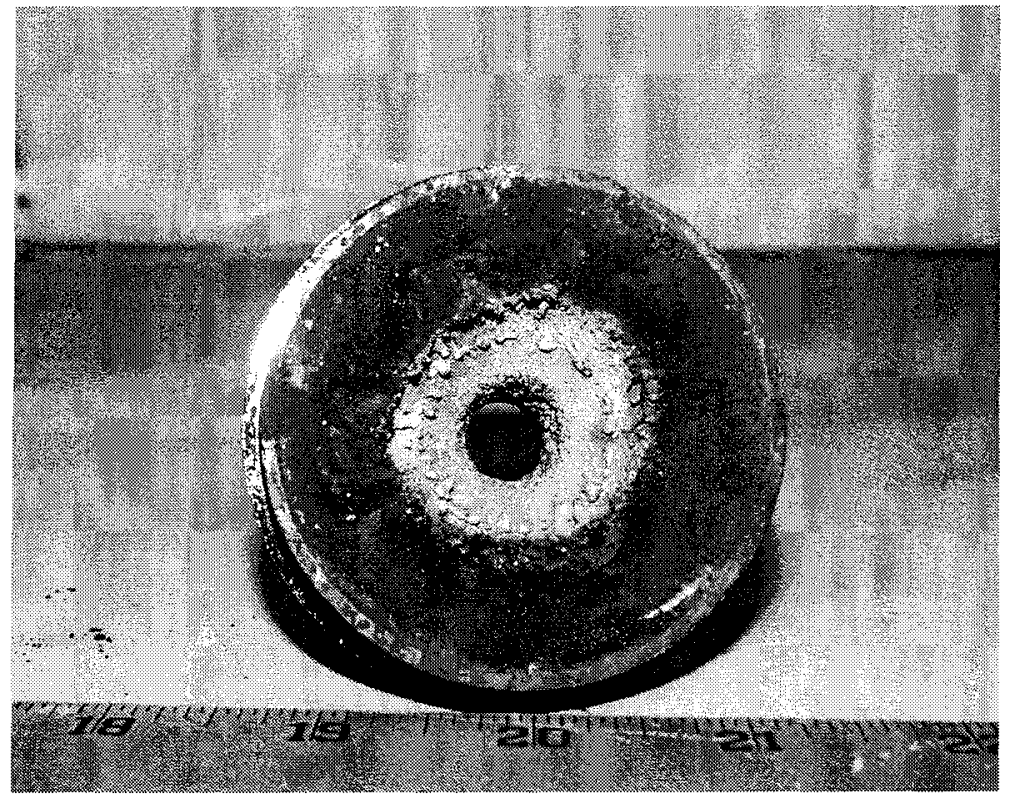

Figure 2.16: Slag agglomeration on a nozzle from Test 1.

\section{$60 \% \mathrm{AlMg} / 40 \% \mathrm{HTPB}$ Fuel}

The low pressure tests (Tests 9, 10,13) exhibited some solidified fuel/slag agglomeration on the reaction chamber and fuel grain, however the effect was not as pronounced as with the $60 \% \mathrm{Al} / 40 \% \mathrm{HTPB}$ fuel. The high pressure tests (Tests 11, 12) 


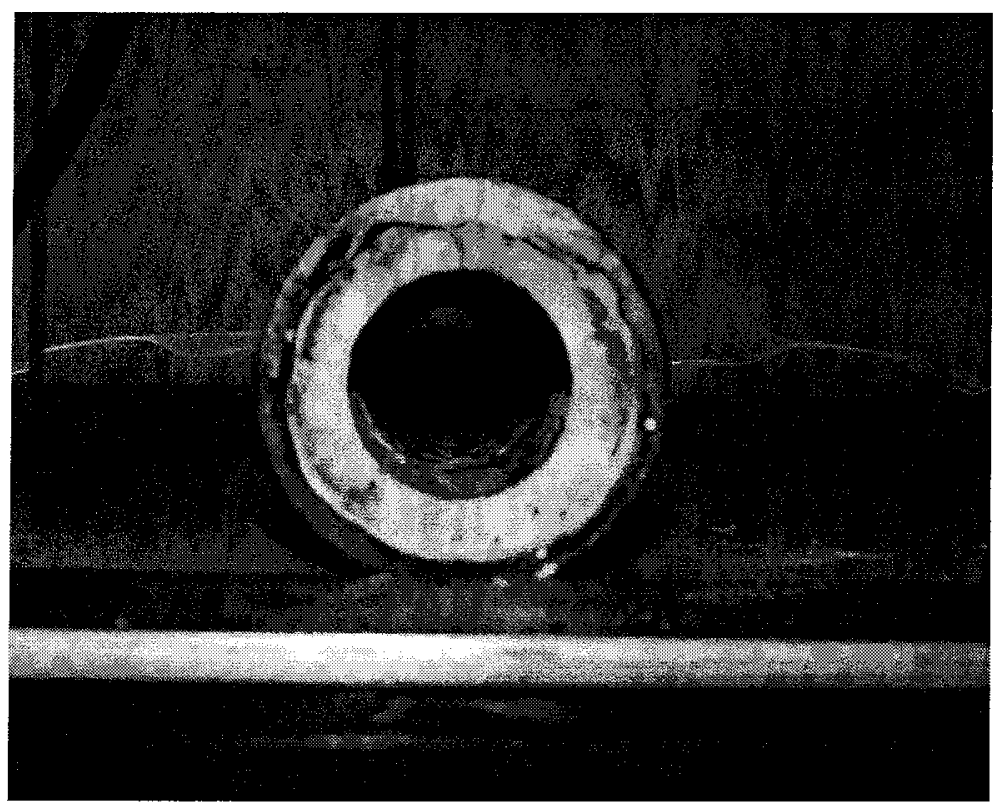

Figure 2.17: Aluminum oxide powder on a spent fuel grain.

showed a very clean grain burn surface and almost no agglomeration on any motor components. Chamber pressure fluctuations on the order of $2 \%-5 \%$ of mean pressure were observed indicating stable combustion.

\section{HTPB Fuel}

Both tests showed a clean grain surface after firing and no material agglomeration on any motor components. Chamber pressure fluctuations on the order of $2 \%-5 \%$ of mean pressure were observed indicating stable combustion.

Figure 2.18 shows the nozzles from Tests 3,10 and 15 along with a new nozzle for reference. The decrease of slag agglomeration with the AlMg fuel versus the $\mathrm{Al}$ fuel and the complete lack of any material agglomeration with the HTPB 
fuel is evident. Note that some of the agglomerated material on the nozzles from

Tests 3 and 10 was inadvertently removed during the disassembly of the motor.

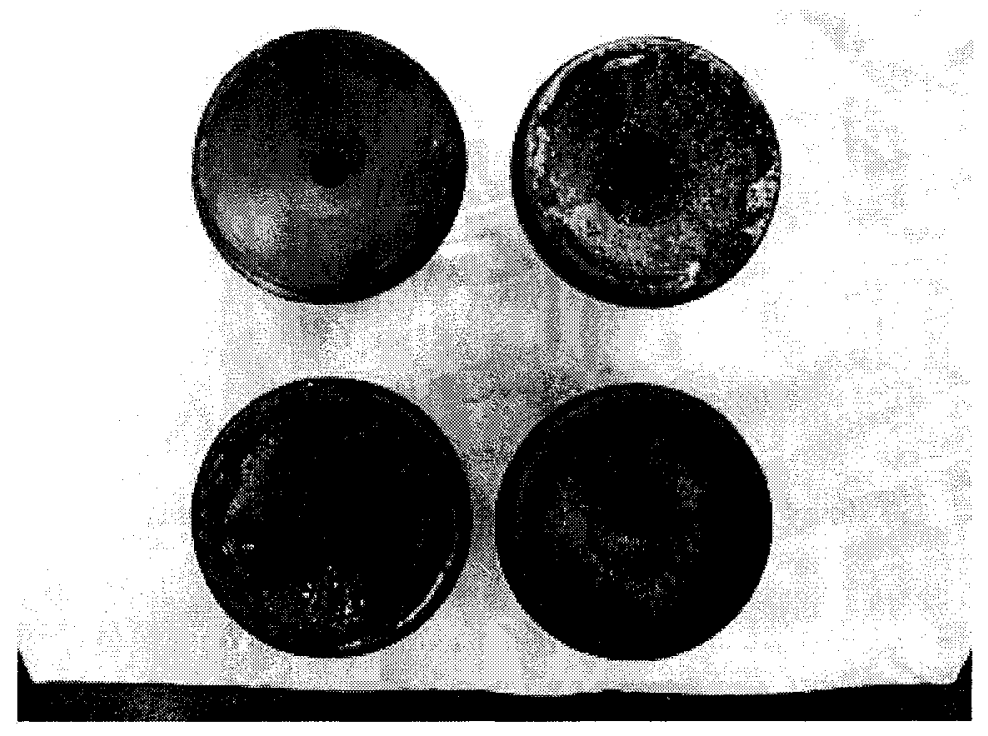

Figure 2.18: Hyper $\mathrm{G}$ nozzles with slag agglomeration. From left to right, top to bottom: new, Test 3, Test 10, Test 15 .

\subsubsection{Combustion instability}

Combustion instability was apparent in Tests $1-5$, and was approximately quantified as follows:

Test 1: random instability throughout burn duration with approximately 10 psi peak to peak amplitude.

Test 2: instability for approximately the last 6 seconds of motor firing with an approximate frequency and amplitude of $4 \mathrm{~Hz}$ and 70 psi peak to peak, respectively.

Test 3: instability for approximately the last 5 seconds of motor firing with an 
approximate frequency and amplitude of $4 \mathrm{~Hz}$ and 40 psi peak to peak, respectively. Test 4: instability for approximately the last 3 seconds of motor firing with an approximate frequency and amplitude of $3 \mathrm{~Hz}$ and 50 psi peak to peak, respectively. Test 5: instability for approximately the last 3 seconds of motor firing with an approximate frequency and amplitude of $4 \mathrm{~Hz}$ and 50 psi peak to peak, respectively.

The mean chamber pressure fluctuations in all tests subsequent to Test 5 were $3 \%-5 \%$ of nominal, except for the transient anomalies discussed previously.

It is pertinent to note that there was no possibility of feed system "chugging" in the test assembly due to the high stiffness of the stainless steel construction, and the location of the cavitating venturi that prevented interaction between the pressure fluctuations in the combustion chamber and that of the feed system. The instability appeared to be independent of oxidizer mass flux, and the thrust oscillations were in phase with the chamber pressure oscillations indicating that the instability was not a result of plugging of the nozzle throat with slag or otherwise unreacted material. It should be noted that although the oscillations observed during Test 2 had the highest absolute amplitude of the five tests, the relative magnitude of the oscillations to the average chamber pressure in the lower pressure Test 5 was the largest of any test, indicating that the combustion instability was greater at lower chamber pressures. It was also noted that the magnitude of the instability increased with burn time 
in all tests while the chamber pressure decreased, indicating a relationship between chamber pressure and combustion instability in the experiment.

In Tests 1-5, there were visible pieces of ejected matter in the exhaust of the motor. Neither the pure HTPB nor the $60 \% \mathrm{AlMg} / 40 \% \mathrm{HTPB}$ fuel exhibited any instability at any chamber pressure. These facts, combined with the reasoning discussed above led to the conclusion that the instabilities were a result of "chuffing"; periodic shedding of accumulated molten $\mathrm{Al}_{2} \mathrm{O}_{3}$ from the grain surface and virgin fuel being ignited[30].

The characteristic frequency of the fuel grain was calculated using Equation 2.21 taken from [30] with an approximation of the thermal diffusivity for the $\mathrm{Al} / \mathrm{HTPB}$ fuel combination based on molar averages of the major constituents of the fuel [31]. The resulting frequency was approximately $2 \mathrm{~Hz}$; lower than the $3-4 \mathrm{~Hz}$ frequency observed in these tests. However, the thermal diffusivity for aluminum was taken at $21^{\circ} \mathrm{C}$ while the combustion chamber temperature is obviously much higher. As thermal diffusivity decreases with increasing material temperature, the predicted frequency from Equation 2.21 was likely higher than that calculated, presumably closer to the observed test frequencies.

$$
f_{\text {chuffing }}=\frac{\dot{r}^{2}}{\alpha}
$$

It was hypothesized that a turbulator insert, acting like a forward facing step, 
with a port diameter smaller than that of the grain port diameter placed upstream of the grain entrance would induce recirculation and therefore increase combustion stability and heat transfer to the grain. The result would be a reduction of the build-up of molten $\mathrm{Al}_{2} \mathrm{O}_{3}$ on the grain surface, the periodic shedding of which was likely the cause of the observed combustion instability. It was also possible that an increase in chamber pressure would act to mitigate the combustion instability due to a pressure dependence of the rate of aluminum particle combustion estimated as $t_{\text {burn }} \sim P_{c}^{-0.2}[32]$. Both of these solutions were implemented; the turbulator in Test 6 and an increase in mean chamber pressure in Tests 7 and 8.

Figure 2.19 shows the chamber pressure traces from Test 3 (indicating severe combustion instability), Test 6 (with turbulator) and Test 7 (with increased chamber pressure). The traces were lagged shifted in time on the figure for ease of readability. The addition of the turbulator clearly eliminated the combustion instability. It appeared that the increase in chamber pressure also eliminated the combustion instability. However, because the chamber pressure was increased by nearly doubling the oxidizer mass flow and therefore the mass flux by using a venturi with a larger throat diameter in this experiment, it was likely that the elimination of combustion instability in Test 7 was not due to a chamber pressure increase. The higher flow velocity relative to the grain and larger quantity of pyrolyzation gases liberated due to the increase in mass flux may acted to reduce the thickness of the layer of molten 
$\mathrm{Al}_{2} \mathrm{O}_{3}$ on the grain surface or remove it from the surface altogether, mitigating or eliminating the combustion instability observed in Test 3.

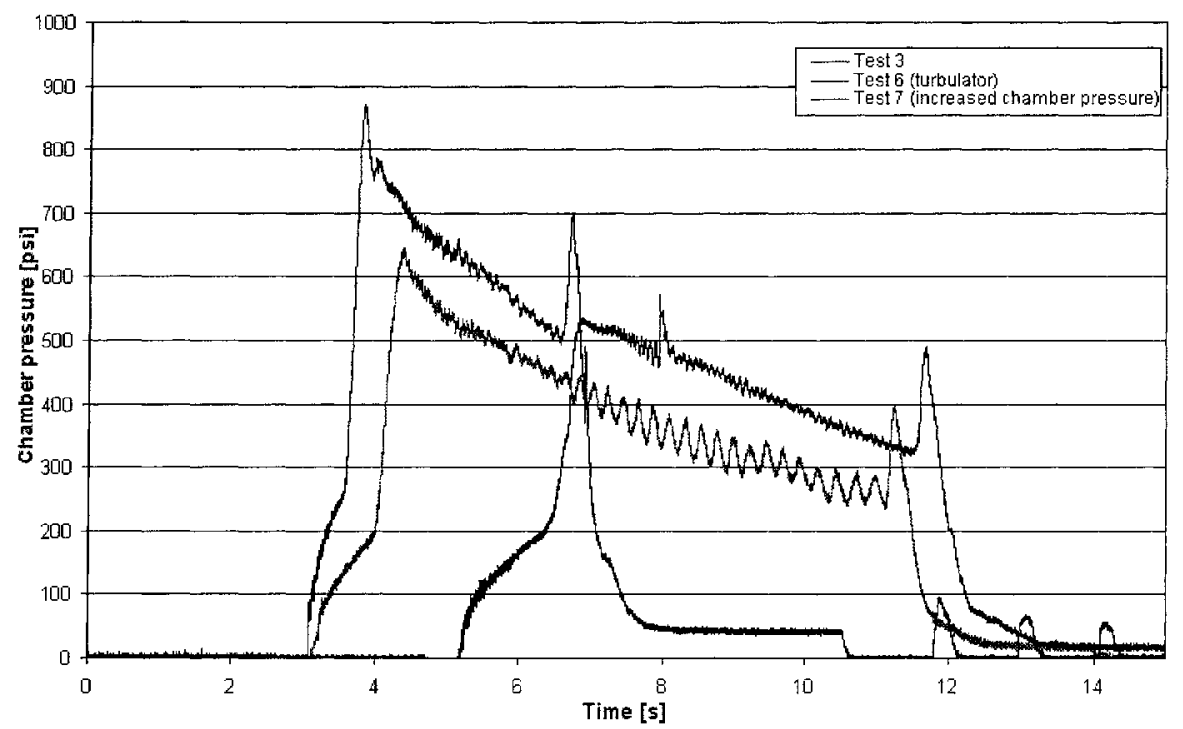

Figure 2.19: Combustion instability in Test 3, elimination of combustion instability in Tests 6,7 .

\subsubsection{Nozzle erosion}

Significant nozzle erosion occurred in Tests 1-5 and as such higher quality graphite (material with higher density) was used to manufacture the nozzles for the remaining tests. The marked difference in the graphite ablation rate even with the higher quality graphite for $60 \% \mathrm{Al} / 40 \% \mathrm{HTPB}$ tests relative to the other tests in this study was explained by the higher combustion temperature of the $60 \% \mathrm{Al} / 40 \% \mathrm{HTPB}$ fuel relative to the other fuels, as well as the high concentration of particulate material observed in the exhaust of the $60 \% \mathrm{Al} / 40 \% \mathrm{HTPB}$ combination. Both of these 
characteristics would have increased the erosion rate of the material. Table 2.6 summarizes the measured nozzle erosion as a function of fuel combination, and presents the theoretical combustion temperatures for each combination at an average of the OF ratios tested in this work. The average nozzle erosion during Tests $6-8$ with the higher quality graphite was $0.6 \mathrm{~mm}$ lower than that during Tests 1-5. Extremely high nozzle erosion with highly aluminized fuels was also noted by Lips[11]. In general, work by Bunker[33] indicates that a higher rate of nozzle regression can be expected from hybrid motors than from solid motors, due to the higher concentration of oxygen containing species in the exhaust.

Table 2.6: Nozzle throat erosion for various Fuel/90\%HP propellant combinations.

\begin{tabular}{|l|l|l|l|}
\hline Fuel & Flame Temp. $[\mathrm{K}]$ & Erosion Range $[\mathrm{mm}]$ & Avg. Erosion $[\mathrm{mm}]$ \\
\hline \hline $60 \% \mathrm{Al} / 40 \%$ HTPB & 3356 & $2.4-5.8$ & 3.4 \\
original graphite & & & \\
$60 \% \mathrm{Al} / 40 \% \mathrm{HTPB}$ & 3344 & $2.3-3.7$ & 2.8 \\
$60 \% \mathrm{AlMg} / 40 \% \mathrm{HTPB}$ & 3274 & $0.0-1.1$ & 0.5 \\
$\mathrm{HTPB}$ & 2820 & $0.0-0.8$ & 0.4 \\
\hline
\end{tabular}

\subsubsection{Regression rate}

Figure 2.20 shows the measured regression rate for each fuel combination as a function of average total mass flux, $\overline{G_{t o t}}$. Data from other relevant work is also shown for comparison purposes and references are included in the figure legend. An increase in regression rate was noted with the addition of the magnesium to the fuel 
composition; the regression rate was consistently approximately $0.2 \mathrm{~mm} / \mathrm{s}$ greater at each oxidizer flux level tested than that of the $60 \% \mathrm{Al} / 40 \% \mathrm{HTPB}$ fuel, possibly due to increased reactivity of the $\mathrm{AlMg} / \mathrm{HTPB}$ fuel combination. The test with a turbulator upstream of the fuel grain demonstrated a regression rate increase of the $60 \% \mathrm{Al} / 40 \% \mathrm{HTPB}$ fuel relative to the other tests, as the increased turbulence level resulted in an increase in convective heat transfer to the fuel grain surface. Test 6 exhibited a $0.87 \mathrm{~mm} / \mathrm{s}$ regression rate, almost $0.2 \mathrm{~mm} / \mathrm{s}$ higher than that of Test 3 , which was performed under nearly identical conditions to that of Test 6 without the turbulator insert. Test 6 is shown as a single data point on Figure 2.20, and not included in the correlation presented in Equation 2.22 as the motor geometry for this test varied from that of the baseline configuration.

The regression rates exhibited by the pure HTPB fuel compared well to those given in [3] for a CTPB/96\%HP propellant combination. Although a reference for metallized HTPB fuel grains combusting with HP could not be located, a comparison to the results reported in [11] indicated that the regression rates observed in this work for the $60 \% \mathrm{Al} / 40 \% \mathrm{HTPB} / 90 \% \mathrm{HP}$ combination were consistently approximately half of those reported for $60 \% \mathrm{Al} / 40 \% \mathrm{PU} / \mathrm{FLOX}-40$. The oxidizer flux exponent in the regression rate correlation presented in[11] is shown to be 0.52 on $G_{o x}$ for the $60 \% \mathrm{Al} / 40 \% \mathrm{PU} / \mathrm{FLOX}-40$ propellant combination. In comparison, when performing a correlation like that shown in Equation 2.22 using $\overline{G_{O x}}$ rather 
than $\overline{G_{t o t}}$ as the independent variable, one obtains an exponent of 0.67 for the $60 \% \mathrm{Al} / 40 \% \mathrm{HTPB} / 90 \% \mathrm{HP}$ data presented here. It should be noted that the data and correlation in [11] included only the regression data from the aft $\frac{1}{3}$ length of the fuel grain in an attempt to eliminate the influence of injector effects on the correlations. Additionally, it is pertinent to note that FLOX is a highly toxic and corrosive oxidizer.

The data presented in Figure 2.20 from [7] refer to experiments performed with lithium aluminum hydride/polyethylene fuel and 90\%HP. Lithium aluminum hydride, like hydrides in general, is a pyrophoric and water-reactive compound meaning it will ignite spontaneously in air and reacts violently with water. These characteristics produce the high regression rate relative to the other fuels presented in Figure 2.20. Although not included in Fig. 2.20, it is also pertinent to note that work by Chiaverini et al. [17] showed that the addition of UFAL (ultra-fine aluminum powder) resulted in a regression rate increase of approximately $40 \%$ over baseline HTPB tests with GOX. UFAL particle sizes are typically $0.05 \mu \mathrm{m}-0.10$ $\mu \mathrm{m}$.

A meaningful power law correlation for the regression rate data as a function of total mass flux for both the $60 \% \mathrm{Al} / 40 \% \mathrm{HTPB} / 90 \% \mathrm{HP}$ and $60 \% \mathrm{AlMg} / 40 \% \mathrm{HTPB} / 90 \% \mathrm{HP}$ fuel combinations are given in Equations 2.22 and 2.23 . 


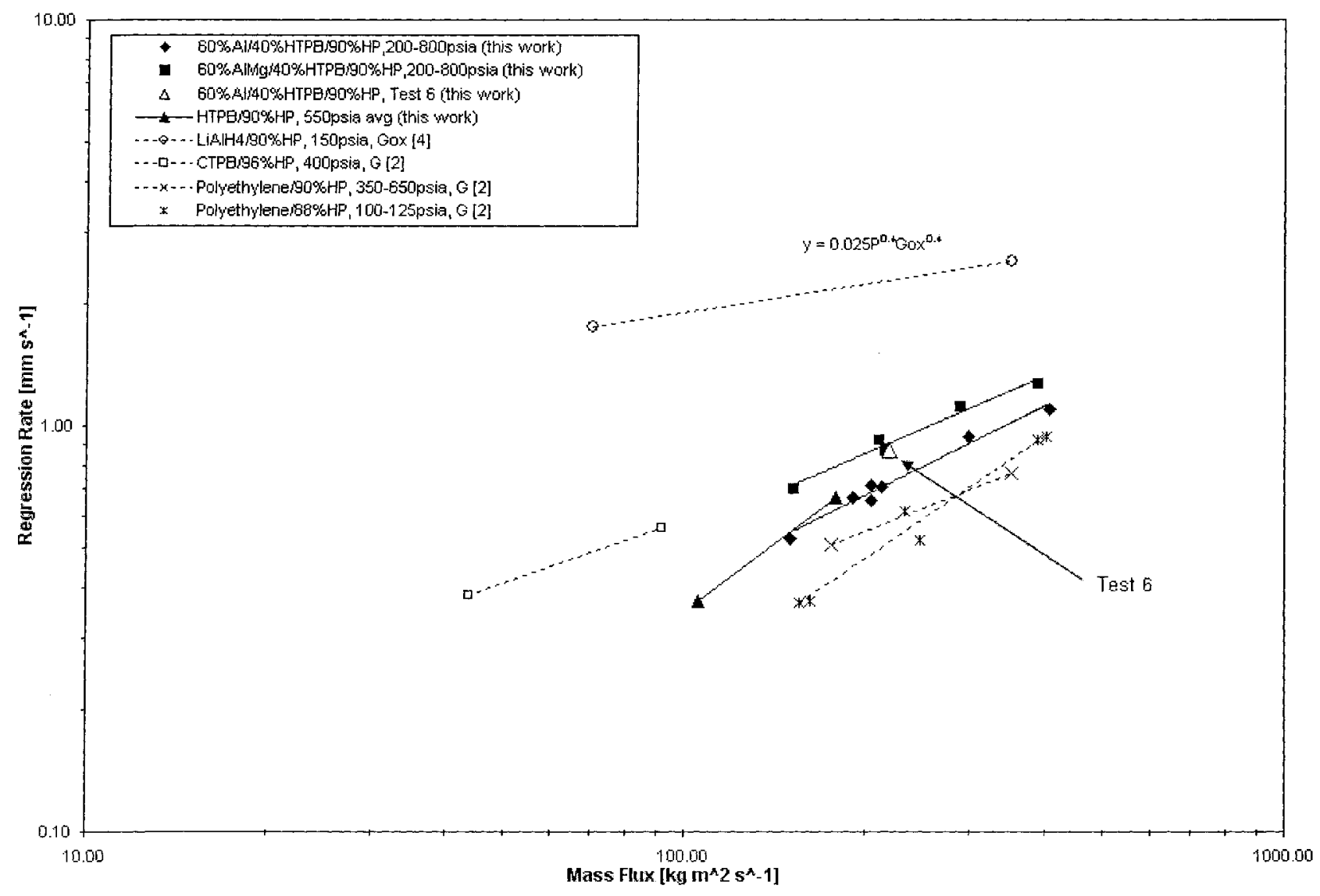

Figure 2.20: Regression rate data from all HyperG tests, comparable data from other experiments using lab scale hybrid motors and HP oxidizer. 
For the $60 \% \mathrm{Al} / 40 \% \mathrm{HTPB} / 90 \% \mathrm{HP}$ combination:

$$
\overline{\dot{r}}=0.014{\overline{G_{t o t}}}^{0.74} \mathrm{~mm} / \mathrm{s}
$$

For the $60 \% \mathrm{AlMg} / 40 \% \mathrm{HTPB} / 90 \% \mathrm{HP}$ combination:

$$
\overline{\dot{r}}=0.029{\overline{G_{t o t}}}^{0.64} \mathrm{~mm} / \mathrm{s}
$$

The exponent on the total mass flux is less than the theoretical value of 0.8 expected for pure convective heat transfer in a turbulent boundary layer over a flat plate (refer to Section 1.2) indicating that radiation likely plays a role in the combustion of these propellant combinations, as is expected for a highly metallized fuel grain. This correlation does not attempt to account for possible injector or motor geometry effects. Data was not obtained for multiple chamber pressures at fixed oxidizer mass flux levels with this motor hardware therefore any dependence of regression rate on combustion pressure could not be explicitly identified with the results of the tests performed.

\section{Influence of pressure on regression rate}

There exists some experimental data to support a dependence of regression rate on chamber pressure due to a dependence of radiative heat transfer on chamber pressure in laboratory scale HTPB/GOX motors, see [34] for example. A quantitative reason 
for this dependence is presented in Equation 3.48, which describes the exponential dependence of gas absorption coefficient on pressure. A general form of a regression correlation applicable to motors exhibiting pressure dependent regression rates due to a significant radiative contribution to fuel surface heat flux was presented in Equation 1.24. Pressure dependencies of this type would apply to motors with significant portions of soot in the combustion chamber or metal additives in the fuel grain. The correlation obtained in [11] for the metallized $60 \% \mathrm{Al} / 40 \% \mathrm{PU} / \mathrm{FLOX}-40$ propellant combination indicated a dependence of regression rate on pressure.

A definitive pressure dependence of fuel regression rate was noted in [15] and [16] for a lithium hydride based fuel grain with a FLOX oxidizer, and various non-metallized polymer fuels operating with a FLOX oxidizer. The pressure dependencies were observed at mass flux $>70 \mathrm{~kg} / \mathrm{m}^{2}$ s and low pressures, $<150 \mathrm{psia}$, and were attributed to a heterogeneous rate limiting kinetic reaction at the oxidizer/fuel interface.

An illustration of the regression rate regimes where a pressure dependence is predicted for a hybrid rocket motor is presented in Figure 2.21 taken from [35]. Note that the axes ranges are not labeled, as the existence and extent of each regime would vary with fuel formulation. Portions of this figure have been experimentally explored for various fuels (for example in References [15] and [16]) but a complete picture of the regression rate functionality has not been produced over large pressure 
and mass flux ranges for any fuel to date.

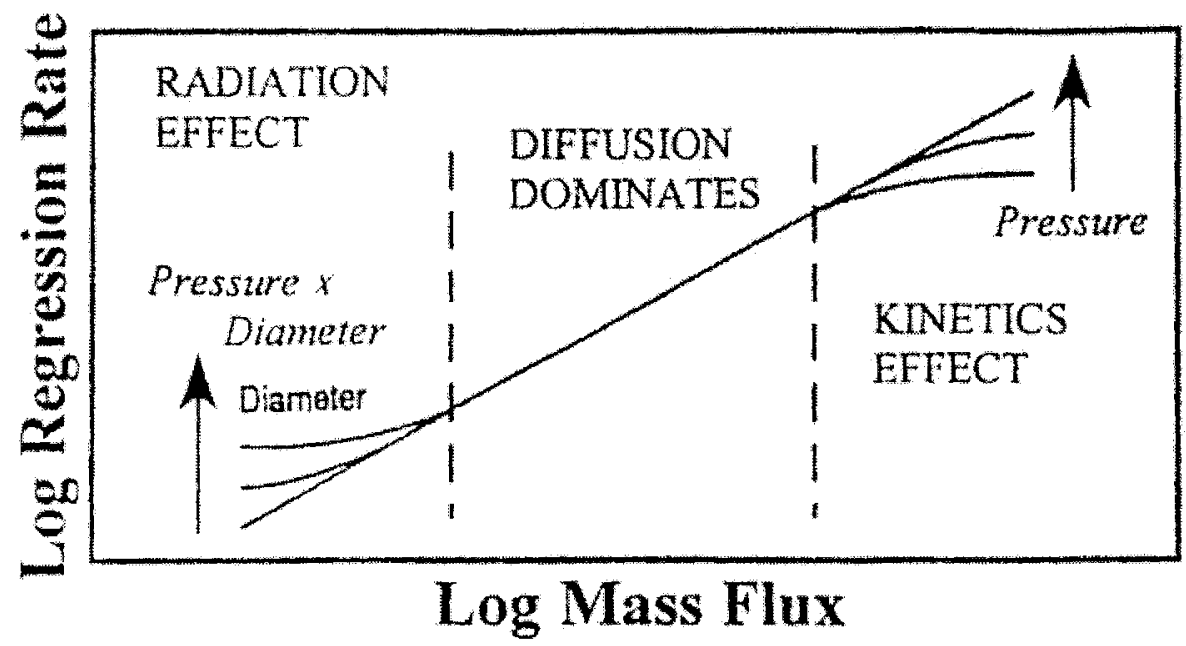

Figure 2.21: Qualitative illustration of the effects of pressure and total mass flux variations on the regression rate in a hybrid motor.

\subsubsection{Combustion efficiency}

Tests 1 and 2 did not utilize a reaction chamber downstream of the fuel grain and exhibited large amounts of slag agglomeration on the nozzle and fuel, as well as significant amounts of ejected matter in the motor exhaust. These phenomena are indicators of incomplete combustion of the reactants. A common measure of the effective combustion length for a motor is the characteristic length, $L^{*}$. Tests 1 and 2 had an effective characteristic length of zero as they did not have a reaction chamber. In all subsequent tests, a reaction chamber was employed bringing the initial motor characteristic length to $L^{*}=1.6 \mathrm{~m}-4.3 \mathrm{~m}$. In the experiments per- 
formed in [3], a correlation between characteristic length and combustion efficiency for polyethylene/ $85 \% \mathrm{HP}$ gave the following results in lab scale motors, providing a baseline for the $\overline{\eta c^{*}}$ range expected in the HyperG tests.

$$
\begin{aligned}
& L^{*}=1.5 m, \eta c^{*}=0.90-0.98 \\
& L^{*}=3.0 m, \eta c^{*}=0.92-1.00
\end{aligned}
$$

Table 2.7 shows a summary of the combustion efficiencies obtained for each propellant combination. Note that the average combustion efficiency for Tests 1 and 2 was lower than that of the other $60 \% \mathrm{Al} / 40 \% \mathrm{HTPB}$ tests and that data has been separated from the data for Tests 3-8. Due to the large amount of nozzle throat regression prevalent in the $60 \% \mathrm{Al} / 40 \% \mathrm{HTPB}$ test data and subsequent overestimation of the value for $\overline{\eta c^{*}}$ using Equation 2.15 , the estimated value of $\overline{\eta c_{e s t}^{*}}$. from Equation 2.17 was used in the analysis of the data for Tests 1-8.

It was hypothesized that the low combustion efficiency of the $60 \% \mathrm{Al} / 40 \% \mathrm{HTPB}$ fuel combination was a result of incomplete combustion of the aluminum due to the formation of aluminum oxide $\left(\mathrm{Al}_{2} \mathrm{O}_{3}\right)$ around aluminum particles during the combustion process, inhibiting mass and heat diffusion to the interior of the particle. This effect has been recorded in literature repeatedly [7], [11]. Due to the hypothesis 
Table 2.7: Summary of combustion efficiencies of various Fuel/90\%HP combinations and data from other relevant lab scale hybrid motor experiments.

\begin{tabular}{|c|c|c|}
\hline Fuel & $\overline{\eta c^{*}}$ Range & $\overline{\eta c^{*}}$ Average \\
\hline $60 \% \mathrm{Al} / 40 \% \mathrm{HTPB}$, Tests 1,2 & $0.72,0.74$ & 0.73 \\
\hline $60 \% \mathrm{Al} / 40 \% \mathrm{HTPB}$ & $0.73-0.89$ & 0.83 \\
\hline $60 \% \mathrm{AlMg} / 40 \% \mathrm{HTPB}$ & $0.74-0.87$ & 0.81 \\
\hline HTPB & $0.79,0.90$ & 0.85 \\
\hline $60 \% \mathrm{Al} / 40 \% \mathrm{PU} / \mathrm{FLOX}-40[11]^{a}$ & - & 0.92 \\
\hline $80 \% \mathrm{Al} / 20 \% \mathrm{PU} / \mathrm{FLOX}-40[11]$ & - & 0.89 \\
\hline $60 \% \mathrm{Al} / 40 \% \mathrm{PU} / \mathrm{LOX}[11]$ & - & 0.81 \\
\hline Polyethylene $/ 88 \% \mathrm{HP}[4]^{b}$ & $0.87-0.98$ & 0.93 \\
\hline $95 \% \mathrm{LiAlH}_{4} / 5 \%$ polyethylene $/ 90 \% \mathrm{HP}[7]$ & $0.79-0.86$ & 0.83 \\
\hline
\end{tabular}

${ }^{a}$ The motors used in this test utilized a mixing diaphragm (similar to the turbulator used in this work) in the reaction chamber to increase the combustion efficiency. The values shown here are the uncorrected values, they have not been adjusted for the author's estimate of efficiency losses due to motor heat loss, nor that due to increased mass flow from $\dot{m}_{\text {inert }}$.

"The authors note that significant nozzle erosion occurred during these tests, and may have effected the calculation of $\overline{\eta c^{*}}$.

that magnesium combusts in the gaseous phase [36], the combustion efficiency of the magnesium/aluminum alloy fuel, used in Tests 9 through 13, was expected to be higher than that of the aluminum fuel combination. However, the much larger particle size of the $\mathrm{Al} / \mathrm{Mg}$ alloy used in the fuel for those tests may have contributed to the inefficient combustion. The increased combustion time of the larger diameter particles relative to the chamber residence time [32] may have offset the gains of utilizing magnesium in the fuel grain. Furthermore, the exhaust of the $60 \% \mathrm{AIMg} / 40 \% \mathrm{HTPB}$ fuel combination contained two solid constituents: $\mathrm{MgO}$ and $\mathrm{MgAl}_{2} \mathrm{O}_{4}$. Failure of these oxides to condense completely in the combustion chamber would have resulted 
in a lower combustion temperature due to the loss of the latent heat of fusion from these reactions [12].

The incomplete combustion of the aluminum and aluminum/magnesium alloy particles in the combustion chamber contributed to low ISP efficiency as, in addition to a lowered combustion temperature, high two-phase flow losses due to unreacted fuel were expected in the nozzle. The change of area ratio and therefore the change of back pressure at the nozzle exit due to throat and exit diameter erosion, as well as the subsequent roughening of the surface of the graphite in the nozzle throat, had a reducing effect on the $\overline{\eta I S P}$ term. These latter two effects are estimated in [12] to account for $2.5 \%$ of the reduction in $\overline{\eta I S P}$. Lastly, heat loss from the motor casing during each test contributed to the loss of combustion efficiency; this effect was estimated at $2.0 \%$ in [12] on both the $\overline{\eta I S P}$ and $\overline{\eta c^{*}}$ terms.

A source of data regarding the combustion efficiency of HTPB $/ 90 \%$ HP has not been located for comparison to the results of these tests. The HTPB tests were conducted to provide a baseline for comparison to the metallized fuel grains, and more tests are required to give a reliable indication of the combustion efficiency of this fuel. Significant amounts of data exist for polyethylene/HP, and excerpts are shown in Table 2.7. Note that the motor used for the polyethylene/88\%HP tests described in [4] utilized a pellet catalytic bed while this work utilized a silver screen catalytic bed, further differentiating the two motor configurations. 
Data regarding the combustion efficiency of metallized fuel grains in hybrid operation was included in Table 2.7 for a tri-propellant combination utilizing FLOX oxidizer. It should be noted that in general an increase in combustion efficiency with increasing fluorine content of the oxidizer were observed in the work referenced, due to a more favorable composition of the exhaust [11]. The data for $95 \% \mathrm{LiAlH}_{4} / 5 \%$ polyethylene $/ 90 \% \mathrm{HP}$ from [7] showed combustion efficiencies comparable to those measured in this experiment.

Scaling effects have not been considered in this work, and previous work in this area has found that scaling effects are not negligible for laboratory scale motors, nor for motors with a large particulate concentration in the exhaust products [7], [37]. It is important to note that the HP was stored for approximately one year at $70^{\circ} \mathrm{F}-80^{\circ} \mathrm{F}$ temperatures, and a typical decomposition rate for this environment is slightly greater than $1 \%$ per year [27]. Calculations using PROPEP showed that this decrease in HP concentration would produce an estimated $1 \%$ increase in the $\overline{\eta \overline{I S P}}$ and $\overline{\eta c^{*}}$ values obtained for the HTPB/90\%HP propellant combination, and this increase was representative of that expected for the other propellant combinations tested.

Figures $2.22,2.23$ and 2.24 show the combustion efficiency as a function of the average OF ratio for each test. Due to the variation of motor geometry in Tests 1 and 2 from the baseline motor configuration, these data were included in a separate 
data set in Figure 2.22. The measured combustion efficiency appears to be higher when the constituents are not at stoichiometric conditions, and especially when the motor is running oxidizer rich. Qualitatively this is due to the fact that the fuel is completely consumed in a slightly oxidizer rich environment. Similar effects of OF ratio on combustion efficiency were reported in [14]. There was not enough data from Tests 14 and 15 to draw similar conclusions for the HTPB/90\%HP propellant combination. Additional data spanning the entire OF spectrum is required to completely characterize this effect for all the propellant combinations tested.

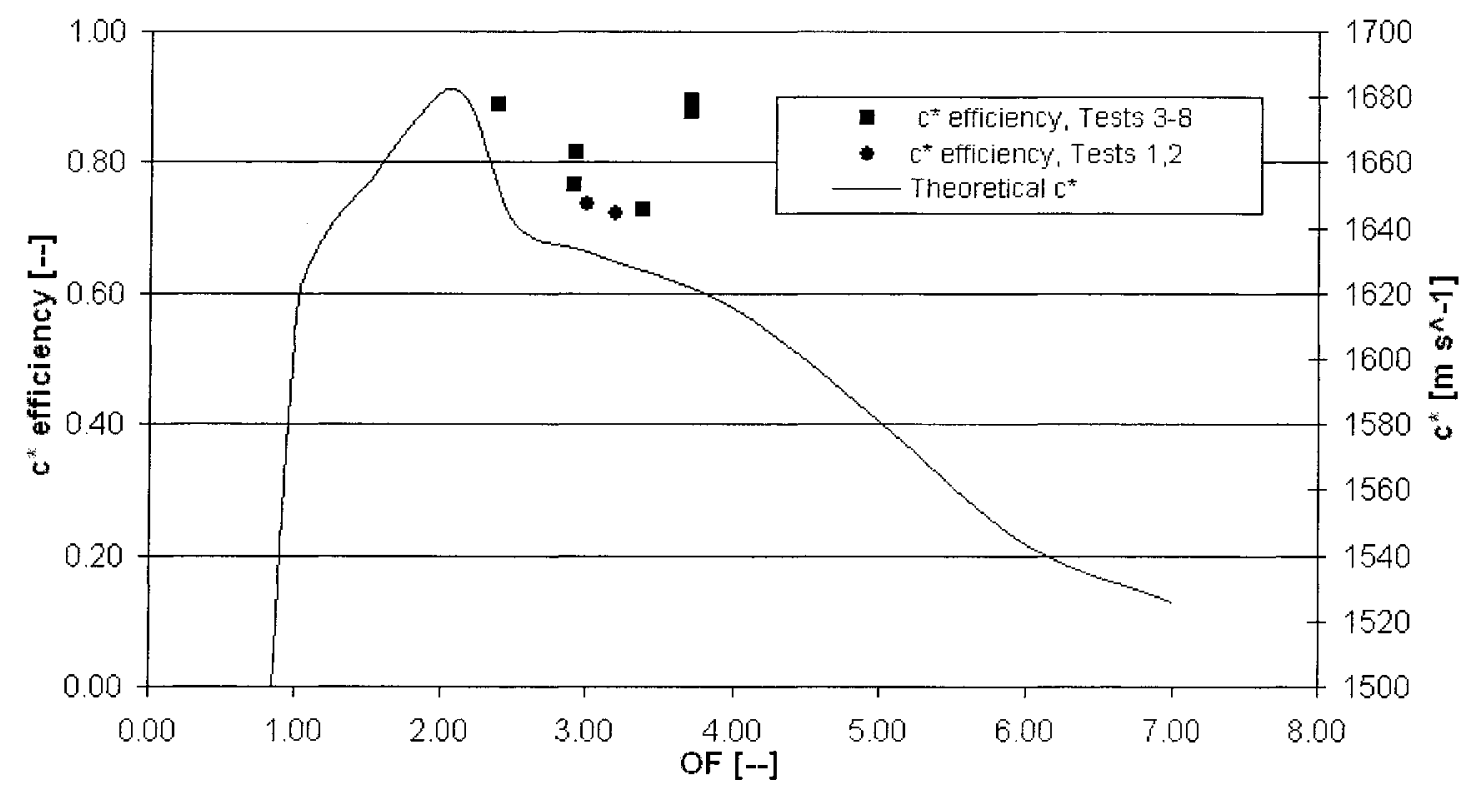

Figure 2.22: Combustion efficiency as a function of $O F$ for $60 \% \mathrm{Al} / 40 \% \mathrm{HTPB} / 90 \% \mathrm{HP}$. 


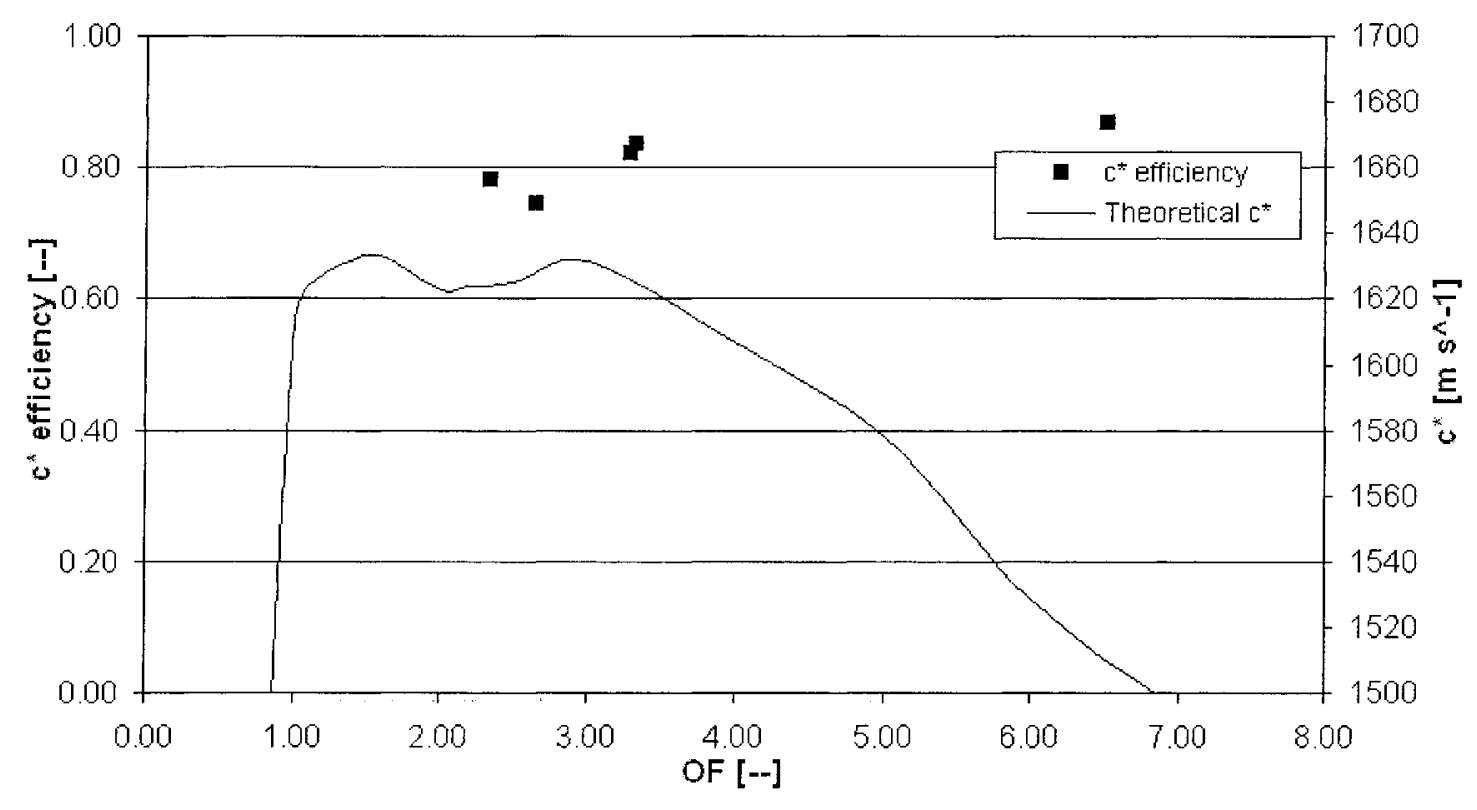

Figure 2.23: Combustion efficiency as a function of $\mathrm{OF}$ for $60 \% \mathrm{AlMg} / 40 \% \mathrm{HTPB} / 90 \% \mathrm{HP}$. 


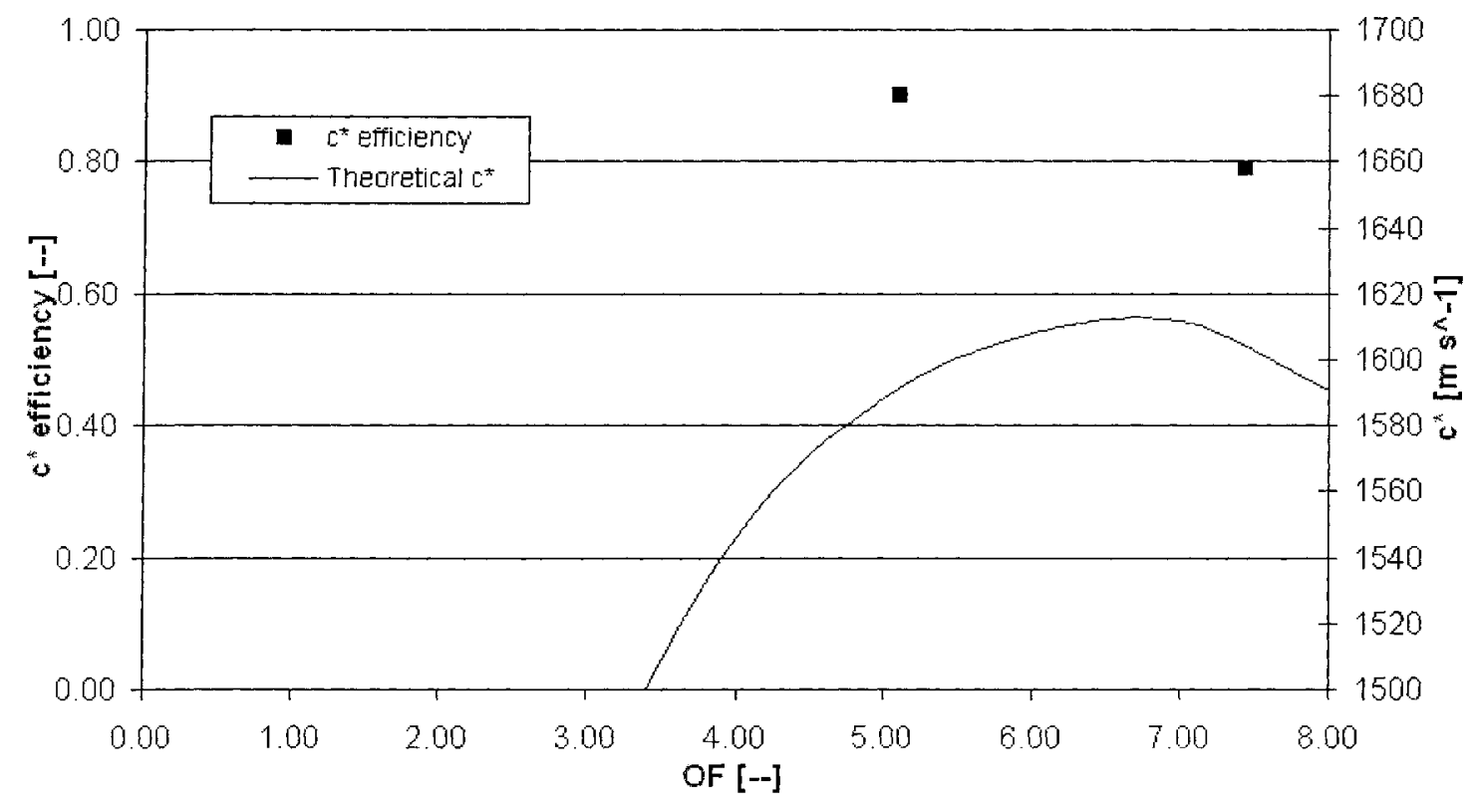

Figure 2.24: Combustion efficiency as a function of OF for HTPB/90\%HP. 


\section{Chapter 3}

\section{Numerical Modeling}

\subsection{Objectives}

Although it is very enjoyable, testing propellant combinations for hybrid rockets is time consuming and costly, as is any other sort of experimental testing. Diagnostics are not always available to allow all parameters of interest to be measured during the test, nor are facilities always available to allow large scale motors to be tested. Numerical models will provide a means by which to gather performance information for hybrid rocket motors while reducing the number of tests required.

A numerical model must be validated with experimental data before the results can be used with any amount of confidence. The model of a hybrid rocket motor is especially complex as it is a transient process, and to obtain a meaningful solution requires the modeling of fluid structure interaction with solid phase heat transfer, resolution of the combusting boundary layer with wall blowing, modeling of 
turbulent flow, turbulent combustion and radiation, and an accurate representation of the incoming flow from the injector, or modeling of the injector component in addition to the chamber. Additionally, the model would span several scales, from retarded flow in the boundary layer to sonic flow at the nozzle throat, to supersonic flow at the nozzle exit. Finally, if fuel with metal additives was to be included in the model then particle transport and multi-phase flow would be added to the list, along with complex burn models for metal particles, multi-component solid models and an increasingly complex combustion model.

Obviously the construction of such a model would be a daunting task. The numerical model described in this chapter was built using many approximations and assumptions that greatly simplified the physics described above. The main goal was to predict the regression rate of the non-metallized HTPB fuel at the start of Tests 14 and 15 of the HyperG program, and to illustrate the interesting characteristics of the hybrid rocket motor flow field: a turbulent boundary layer with blowing and combustion. The model will provide a basic framework on which to add components of increasing complexity, such as: the geometry of the HyperG motor components, moving fluid-solid interfaces, solid phase heat transfer, and solution of the transient flow field. 


\subsection{Model overview}

The numerical model of the hybrid rocket motor (HRM) was constructed using the commercial computational fluid dynamics package CFX Release 5.7.1 at Carleton University. Two models were built, one for each of the HyperG tests conducted with non-metallized HTPB fuel.

In the interest of expanding on the model described herein, the full threedimensional Navier-Stokes equations were solved. However for this first model, a $10^{\circ}$ section of the domain was modeled and symmetry conditions were applied to produce an axisymmetric solution. The spent fuel grains examined during the experiments exhibited an axisymmetric character, supporting the validity of this approximation.

Sources to the continuity equation cannot be applied at a boundary in CFX 5.7.1. In order to simulate the injection of the fuel along the fuel wall, a continuity source term was specified inside a subdomain that had a height equal to the thickness of the first element at the wall, $0.001 \mathrm{~mm}$. The implication of this approximation regarding the physics of the HRM is discussed further in Section 3.3.3.

The solution of the numerical model was completed using only one CPU for various logistical reasons. The geometry of the experimental hardware was simplified, and interfacing the CFX solution to the FORTRAN code to model the spatial variation in the regression rate of the hybrid motor was the primary goal of this model. The precombustion chamber and subsequent forward facing step associated 
with the fuel grain, the reaction chamber, and the nozzle were not included in the model; only the combustion chamber surrounded by the fuel grain was modeled. Similar geometrical simplifications were used by Venkateswaran and Merkle [21]. The implications of the omittance of the precombustion chamber on the prediction of the average regression rate of the fuel are discussed in Section 3.5.4.

A steady-state solution was obtained for each model at the operating conditions present at the start of the respective experimental test. Thus, the results obtained are strictly valid only for the initial steady-state operating conditions of the motor, as the regression of the fuel grain in a hybrid motor causes a change in the flow field characteristics as the test progresses. Additionally, the chamber pressure decreased during the test due erosion of the nozzle throat. Because the fuel surface is constantly regressing in a HRM, strictly speaking the motor is never operating at a steady state. However, the regression rate of the propellant combinations tested was several orders of magnitude lower than the mean velocity in the combustion chamber, and thus the steady-state approximation was valid for a specified set of operating conditions. Experimental verification of this point is given by Stamatov et al. [38]. 


\subsection{Solution of the HRM model using CFX 5.7.1}

\subsubsection{Governing equations}

The governing equations utilized for the HRM model were the three-dimensional, unsteady, Reynolds Averaged Navier-Stokes (RANS) equations consisting of continuity, momentum, and energy. The system was closed with the ideal gas equation of state and constitutive relations, which were modified to account for varying mixture composition. Refer to Equations 3.1 to 3.5, and for further explanation of individual terms to References [39], [40], [41] and [42]. In Equations 3.1, 3.2, 3.3 and 3.4 the symbology corresponds to standard definitions of Reynolds and Favre averaging.

The motion of the bulk fluid is modeled in CFX using single velocity, pressure, temperature, and turbulence fields [39]. However, because the working fluid had multiple components, N-1 species conservation equations were solved. The species production terms due to chemical reactions were modeled using the combined Eddy Dissipation and Finite Rate Chemistry Model and a simplified two step reaction. The influence of the different fluid components on the solution was felt by the mean fields by virtue of variation in the fluid properties. Turbulence closure of the RANS equations was accomplished using the Shear Stress Transport (SST) turbulence model requiring the solution of two additional turbulence transport equations. Finally, one radiative energy transport equation was solved using the P-1 Radiative Transfer model. Details of the equations and simplifications used 
in each submodel are presented in the next section.

$$
\begin{aligned}
& \frac{\partial \bar{\rho}}{\partial t}+\frac{\partial\left(\bar{\rho} \tilde{u_{j}}\right)}{\partial x_{j}}+S=0 \\
& \frac{\partial\left(\bar{\rho} \tilde{Y}_{i}\right)}{\partial t}+\frac{\partial\left(\bar{\rho} \tilde{u}_{j} \tilde{Y}_{i}\right)}{\partial x_{j}}=\frac{\partial}{\partial x_{j}}\left(\tilde{\Gamma_{i}} \frac{\partial \tilde{Y}_{i}}{\partial x_{j}}\right)-\frac{\partial}{\partial x_{j}}(\underbrace{\overline{\rho Y_{i}^{\prime \prime} u_{j}^{\prime \prime}}}_{\mathbf{I}})+S_{i} \\
& \frac{\partial\left(\bar{\rho} \tilde{u}_{i}\right)}{\partial t}+\frac{\partial\left(\bar{\rho} \tilde{u}_{i} \tilde{u}_{j}\right)}{\partial x_{j}}=-\frac{\partial \bar{p}}{\partial x_{i}}-\frac{\partial}{\partial x_{j}}(\overline{\tau_{i j}}+\underbrace{\overline{\rho u_{i}^{\prime \prime} u_{j}^{\prime \prime}}}_{\mathbf{I I}}) \\
& \frac{\partial\left(\bar{\rho} \widetilde{h_{t o t}}\right)}{\partial t}+\frac{\partial\left(\bar{\rho} \widetilde{h_{t o t}} \tilde{u_{j}}\right)}{\partial x_{j}}=\frac{\partial \bar{p}}{\partial t}-\frac{\partial}{\partial x_{j}}(\overline{q_{j}}-\sum_{i}^{N_{C}} \widetilde{\Gamma_{i}} \tilde{h}_{i} \frac{\partial \tilde{Y}_{i}}{\partial x_{j}}+\underbrace{\overline{\rho u_{j}^{\prime \prime} h^{\prime \prime}}})_{\mathbf{I I I}} \\
& -\frac{\partial}{\partial x_{j}}(\underbrace{\widetilde{u_{j}} \overline{\rho u_{i}^{\prime \prime} u_{j}^{\prime \prime}}}_{\mathbf{I V}}+\underbrace{\tilde{u_{i}} \overline{\tau_{i j}}}_{\mathbf{V}}+\underbrace{\overline{u_{i}^{\prime \prime} \tau_{i j}}}_{\mathbf{V I}}+\underbrace{\frac{1}{2} \overline{\rho u_{j}^{\prime \prime} u_{i}^{\prime \prime} u_{i}^{\prime \prime}}}_{\text {VII }}) \\
& \overline{\tau_{i j}}=-\underbrace{\mu_{b}^{\frac{\partial u_{k}}{\partial x_{k}} \delta_{i j}}}_{\text {VIII }}-\overline{\mu\left(\frac{\partial u_{i}}{\partial x_{j}}+\frac{\partial u_{j}}{\partial x_{i}}\right)} \\
& q_{j}=-\lambda \frac{\partial T}{\partial x_{j}}
\end{aligned}
$$

In the species conservation equation (Equation 3.2) the differential motion of individual fluid components is assumed to be entirely due to concentration gradients 
(Fick's Law). The source term includes the effects of chemical reactions. In the energy equation (Equation 3.4) the "Dufour" and "Soret" effects are neglected (see Reference [41], page 198, for a discussion of the implications of this assumption). The fluctuations in the diffusion coefficient are neglected in the energy and species conservation equations. The fluctuations in the component enthalpy and species mass fractions are neglected in the energy equation. The bulk viscosity is replaced by Stokes' hypothesis, $\mu_{b}=-\frac{2}{3} \mu$.

The following terms were neglected in the implemented form of the governing equations [43]:

1. Term VIII of the stress tensor, $\overline{\tau_{i j}}$, in the momentum equation;

2. Term VIII of the stress tensor, $\overline{\tau_{i j}}$, in the energy equation;

3. Terms V,VI, VII in the energy equation, describing the viscous energy dissipation, molecular diffusion, and turbulence transport of kinetic energy, respectively.

The neglect of the dilatation term in the molecular stress tensor is possible because the flow field is of non-isothermal, low speed character, and this term is small relative to the others in the momentum and energy equations. Additionally, it is further assumed that the correlation between the fluctuating viscosity and velocity gradients can be neglected for low speed flows so that Equation 3.5 becomes: 


$$
\overline{\tau_{i j}} \approx-\bar{\mu}\left(\frac{\partial \tilde{u}_{i}}{\partial x_{j}}+\frac{\partial \tilde{u_{j}}}{\partial x_{i}}\right)
$$

The neglect of Term $\mathrm{V}$ in the energy equation is justified by the fact that in a turbulent flow, the Reynolds stresses are much larger than the molecular stresses. Terms VI and VII are commonly neglected in flows where $\bar{\rho} k<<P$ and therefore $k<<\tilde{h}$, as in this study [44].

\subsubsection{Discretization and solution methodology}

The following subsection is a brief summary of the information in the CFX Reference Manual, Reference [46], and is included for completeness.

A finite volume method is used in CFX to discretize the governing equations for solution on the mesh. The nodes in the mesh are placed at the center of each volume, and the governing equations are integrated over each finite volume such that the relevant quantities are conserved for each volume. The solution variation within a volume is calculated via shape functions that are linear in the parametric coordinates.

The unsteady forms of the governing equations are marched in time to the steady-state solution using First Order Euler Backward differencing. The decoupling of the pressure and velocity fields is overcome using an unstaggered, collocated grid with a second order central difference discretization of the continuity equation 
modified by a fourth order spatial derivative in pressure, a damping term.

A second order accurate discretization of the advection terms in the governing equations is used. Ralph-Newton linearization is used to solve the pressure/density coupling of compressible flows.

The coefficients of each non-linear system of equations (mass and momentum, radiation and energy, species conservation, and turbulence transport) are linearized, and the system is solved using an implicit and iterative incomplete lower upper (ILU) factorization technique accelerated with an Algebraic Multigrid method. Note that the mass and momentum system is solved as a fully coupled equation set.

\subsubsection{Wall functions}

A turbulent boundary layer with combustion and blowing exhibits very different characteristics than that without either. A turbulent boundary layer with blowing exhibits a log-law velocity region that is shifted vertically relative to that without blowing due to the strong streamwise convective acceleration added to the boundary layer near to the wall [47]. A turbulent boundary layer with combustion and blowing exhibits virtually no log-law velocity profile region, and demonstrates large velocity overshoots in the boundary layer in the vicinity of the flame [48]. This phenomenon is shown in Figure 3.1 taken from [48].

It was necessary to maintain a mesh resolution of $y+<1.0$ at the wall to 


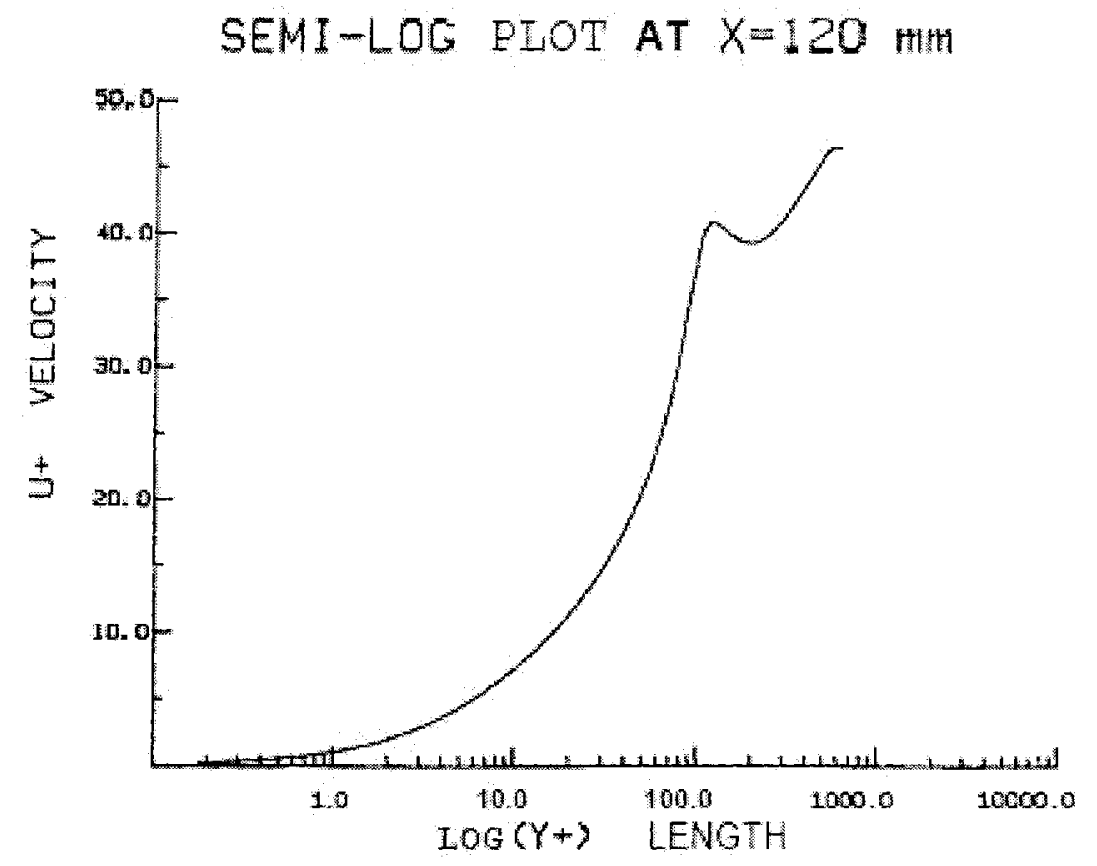

Figure 3.1: Velocity overshoot and lack of log-law velocity profile typical of a turbulent boundary layer with blowing and combustion [48]. 
accurately resolve the temperature and velocity gradients at the wall, and therefore the wall shear stress and wall heat transfer. Doing so kept the first node inside the viscous sub-layer of the boundary layer and therefore did not impose a universal log-law velocity or temperature profile on the flow beyond the first node away from the wall. The form of the wall functions used in CFX for determining the velocity and temperature gradients at the wall in turbulent flow are given in Equations 3.8 and 3.13 and are discussed below [40].

In the overlap or logarithmic region, $\mathrm{y}^{+}>30$, the universal velocity profile is given by the law of the wall:

$$
u^{+}=\frac{1}{\kappa} \ln \left(y^{+}\right)+C
$$

Where $\kappa=0.41$ is the von Kármán constant, $\mathrm{C}=5.0$ and $u^{+}, y^{+}$are defined below.

$$
\begin{array}{r}
y^{+}=\frac{\Delta y u_{\tau}}{\nu} \\
u^{+}=\frac{\tilde{U}_{t}}{u_{\tau}} \\
u_{\tau}=\sqrt{\frac{\tau_{w}}{\bar{\rho}}}
\end{array}
$$

In this notation, $\Delta y$ is spacing of the first node from the surface in the 
surface normal direction and $\mathrm{U}_{t}$ is the known velocity tangential to the surface at the distance $\Delta y$. This form of the universal velocity profile is modified in CFX to allow mesh refinement to very high near wall resolution as is required for the HRM model. The modified equations are given below.

$$
\begin{array}{r}
\tau_{w}=\bar{\rho} u^{*} u_{\tau} \\
u_{\tau}=\sqrt{\nu\left|\frac{\Delta \tilde{U}_{t}}{\Delta y}\right|} \\
u^{*}=\max \left(\sqrt{a_{1} k}, u_{\tau}\right)
\end{array}
$$

Note here that $a_{1}$ is a model constant, and $\mathrm{k}$ is the turbulence kinetic energy.

The universal temperature profile is given as follows (see also Ref. [47]):

$$
T^{+}=\frac{\bar{\rho} C_{p} u^{*}\left(T_{w}-T_{f}\right)}{q_{w}}
$$

Where $\mathrm{T}_{w}, \mathrm{~T}_{f}$ are the wall temperature and fluid temperature at the first node from the wall. The curve fit expression for $\mathrm{T}^{+}$used in CFX 5.7.1 is given below.

$$
T^{+}=\operatorname{Pry}^{*} e^{-\Gamma}+\left[2.12 \ln \left(y^{*}\right)+\beta\right] e^{-\frac{1}{\Gamma}}
$$




$$
\begin{array}{r}
\beta=\left(3.85 \operatorname{Pr}^{\frac{1}{3}}-1.3\right)^{2}+2.12 \ln (\operatorname{Pr}) \\
\Gamma=\frac{0.01\left(\operatorname{Pr} y^{*}\right)^{4}}{1+5 \operatorname{Pr}^{3} y^{*}} \\
y^{*}=\frac{\Delta y u^{*}}{\nu}
\end{array}
$$

In the current model, $\mathrm{y}^{+}<1.0$ and the first node is in the viscous sublayer region. Equation 3.14 reduces to $\mathrm{T}^{+}=\operatorname{Pr} \mathrm{y}^{+}$giving $q_{w}=\lambda \frac{T_{w}-T_{f}}{\Delta y}$. Equation 3.10 reduces to $\tau_{w}=\mu\left|\frac{\Delta \tilde{U}_{t}}{\Delta y}\right|$ because the boundary layer is dominated by viscous shear in this region [47].

The use of subdomain sources to apply the fuel mass injection did not result in the complete inclusion of the effect of wall blowing on wall shear stress and heat transfer, because in the model the fuel injection actually occurred partially at the first node away from the wall. The geometry of a single volume subdomain source along a wall is shown in Figure 3.2 to illustrate this point. In this figure, the mass flow specified for the shaded source would be applied at nodes 1-4 in a control volume-weighted manner, so nodes 3,4 would receive twice the amount of mass injection as nodes 1,2 , since the control volumes associated with those nodes are twice as large [49]. Thus, the near wall velocity and temperature profiles computed in the model were slightly different than those that would have occurred if the entire mass injection term been applied at nodes 1,2 at the wall. Figure 3.3, taken from White[47], illustrates the magnitude of heat transfer variation for a turbulent flat 


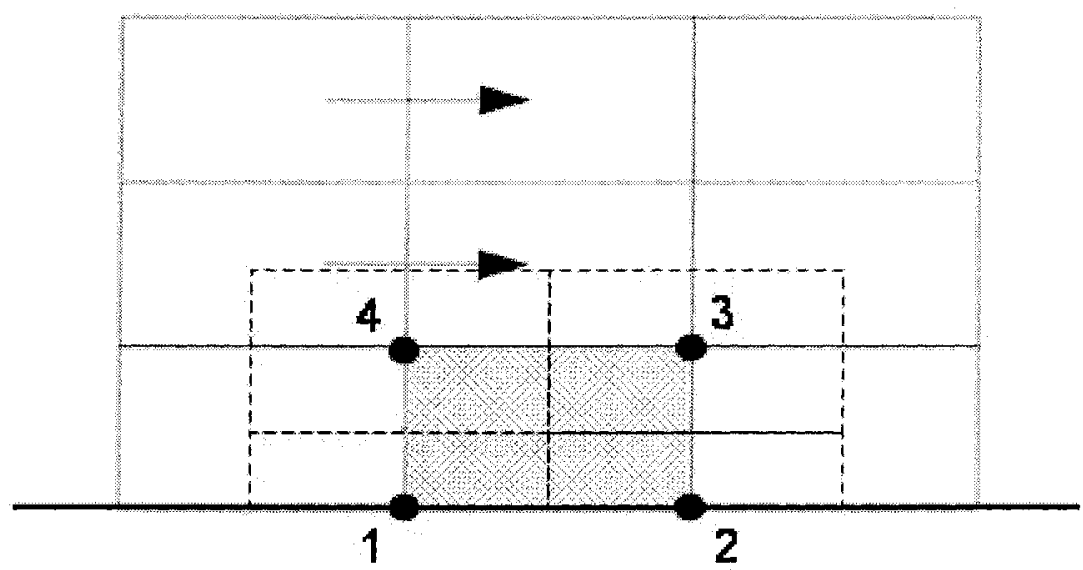

Figure 3.2: Illustration of the application of a subdomain source in CFX 5.7.1. plate flow with blowing or suction. In this figure, $F=v_{w} / U_{e}$ where $v_{w}$ is the transpiration velocity and $U_{e}$ the free stream velocity. For both the HTPB14 and HTPB15 models the parameter $F \sim 0.0005-0.001$.

\subsubsection{Mesh generation and refinement}

The structured, predominantly hexahedral, mesh was generated in ANSYS ICEM CFD Release 5.1. All mesh was generated using to the "Geometric 1" meshing law in ICEM given by Equation 3.18.

$$
h=y_{1} \sum_{x=0}^{n-1} r^{x}
$$

Where $h$ is the total edge length, $r$ is the mesh growth ratio, $y_{1}$ the first node 


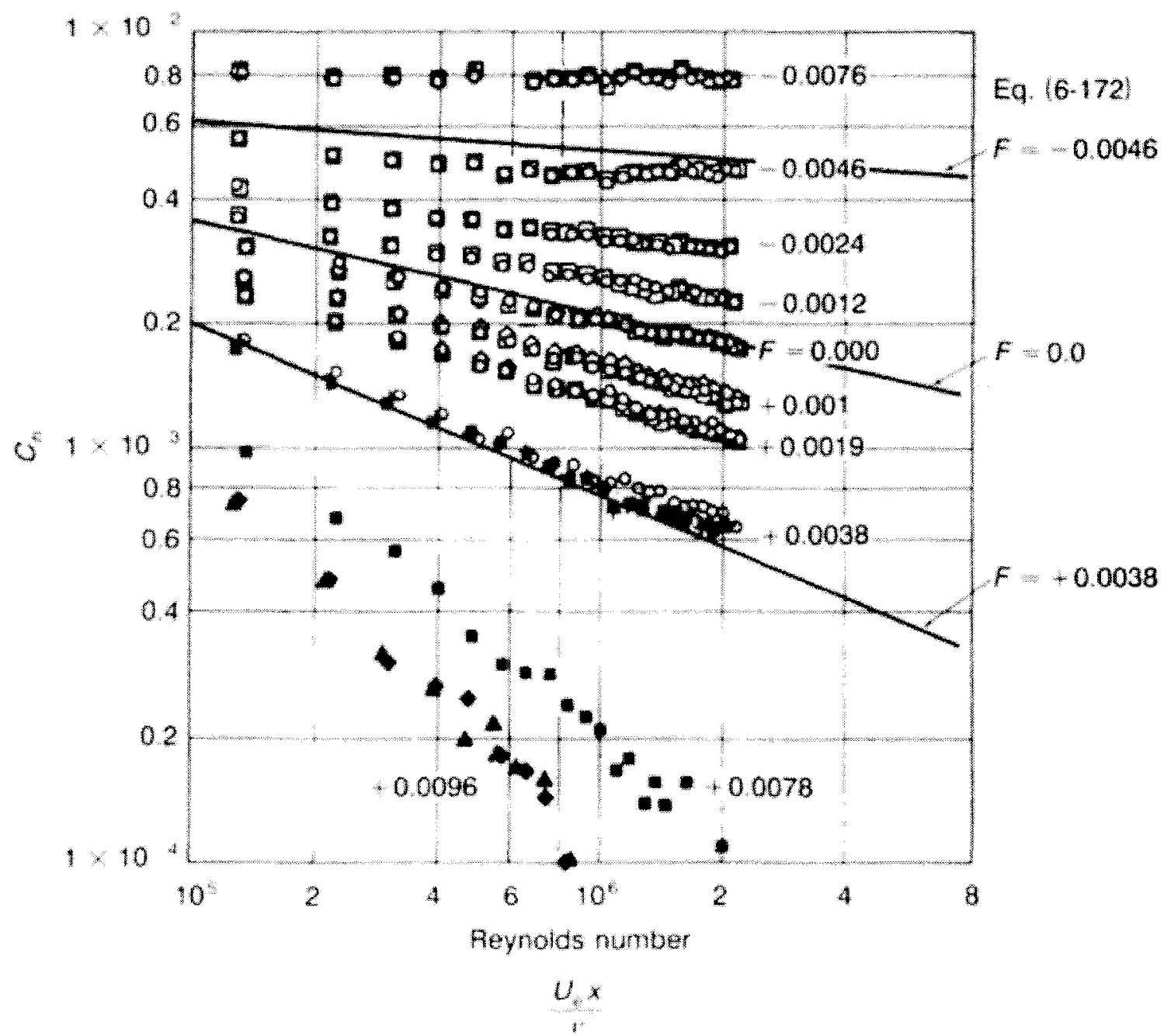

Figure 3.3: Effect of suction or blowing on local Stanton number in a turbulent boundary layer over a flat plate [47]. 
height, and $n$ the number of desired elements along the edge in question.

Three different meshes were generated for the HTPB14 model, nominal, coarse and fine. The statistics for each mesh are summarized in Table 3.1. Figures 3.4 and 3.5 illustrate the change in regression rate and temperature profile at $0.5 \mathrm{~L}$ with mesh refinement. The change in regression rate with mesh refinement was more pronounced than that of the near wall temperature profile, and the regression rate profiles of the nominal and fine mesh are virtually indistinguishable from Figure 3.4. Figure 3.6 shows the change in regression rate for each source relative to that obtained with the nominal mesh. It is evident from this figure that the largest variation in regression rate from the nominal to fine mesh was $0.009 \mathrm{~mm} / \mathrm{s}$, which was less than the experimental error on the $\overline{\dot{r}}$ parameter for Test 14 of $0.015 \mathrm{~mm} / \mathrm{s}$. Thus, the nominal mesh was sufficient for the solution of the HTPB14 model, and the mesh for the HTPB15 model was scaled such that it had the same number of nodes per unit port height, and the same first node spacing as the HTPB14 model. Table 3.2 lists the final mesh statistics for both models. Figure 3.7 shows a portion of the mesh as well as the coordinate axes for the Test 14 model (HTPB14). The entire mesh could not be clearly shown due to the very small near wall spacing. 


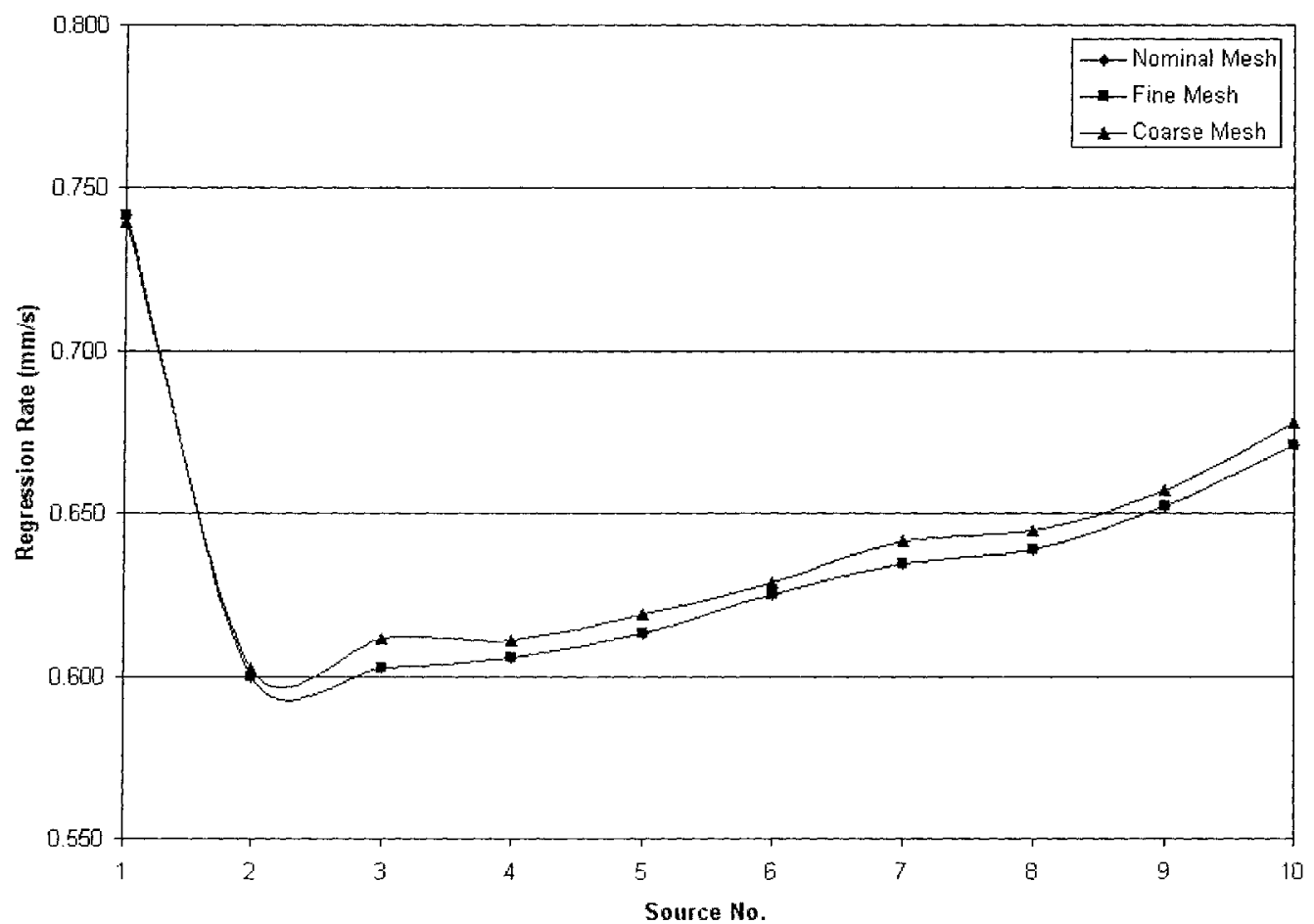

Figure 3.4: Change in regression rate along the length of the grain as the mesh was refined. 


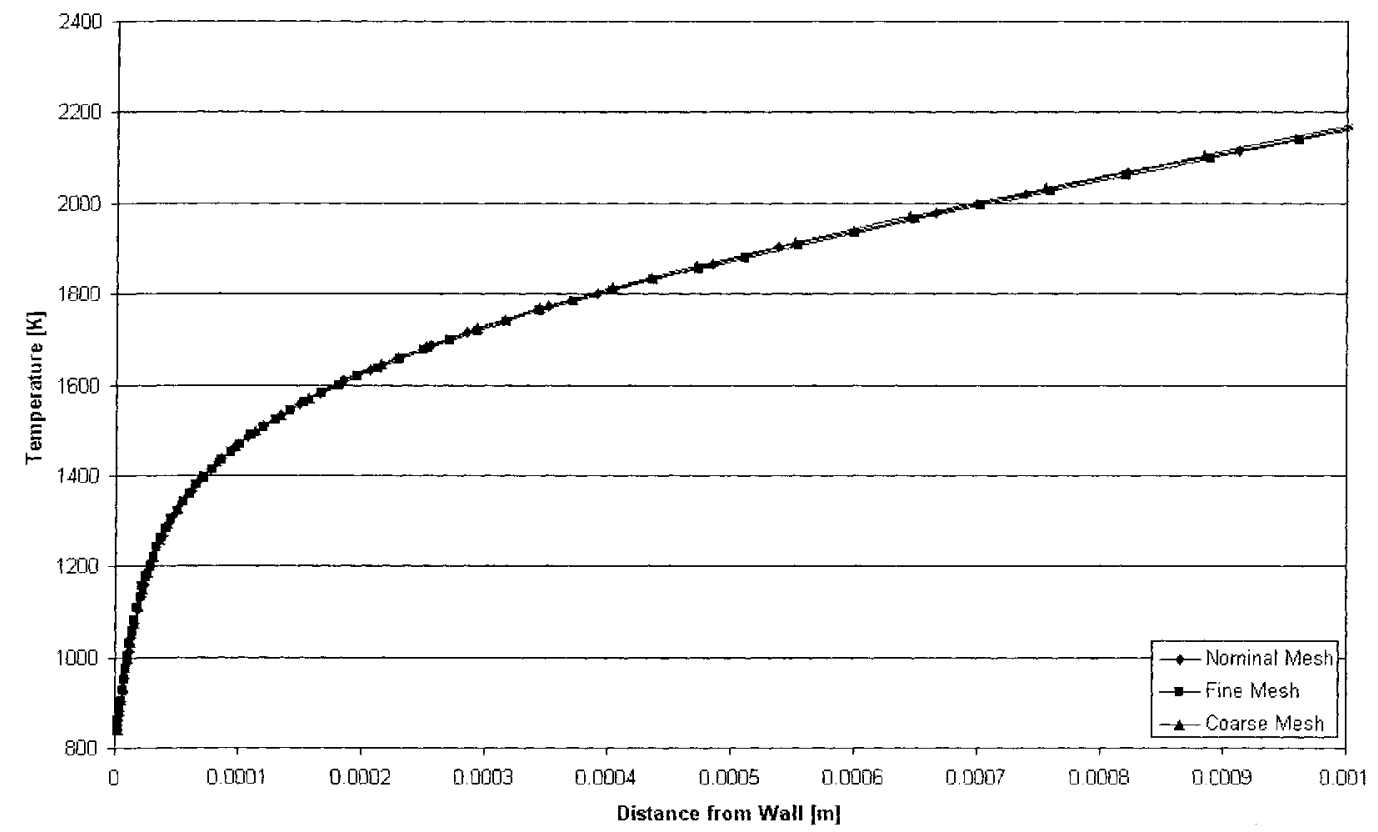

Figure 3.5: Change in temperature profile at $0.5 \mathrm{~L}$ as the mesh was refined. 


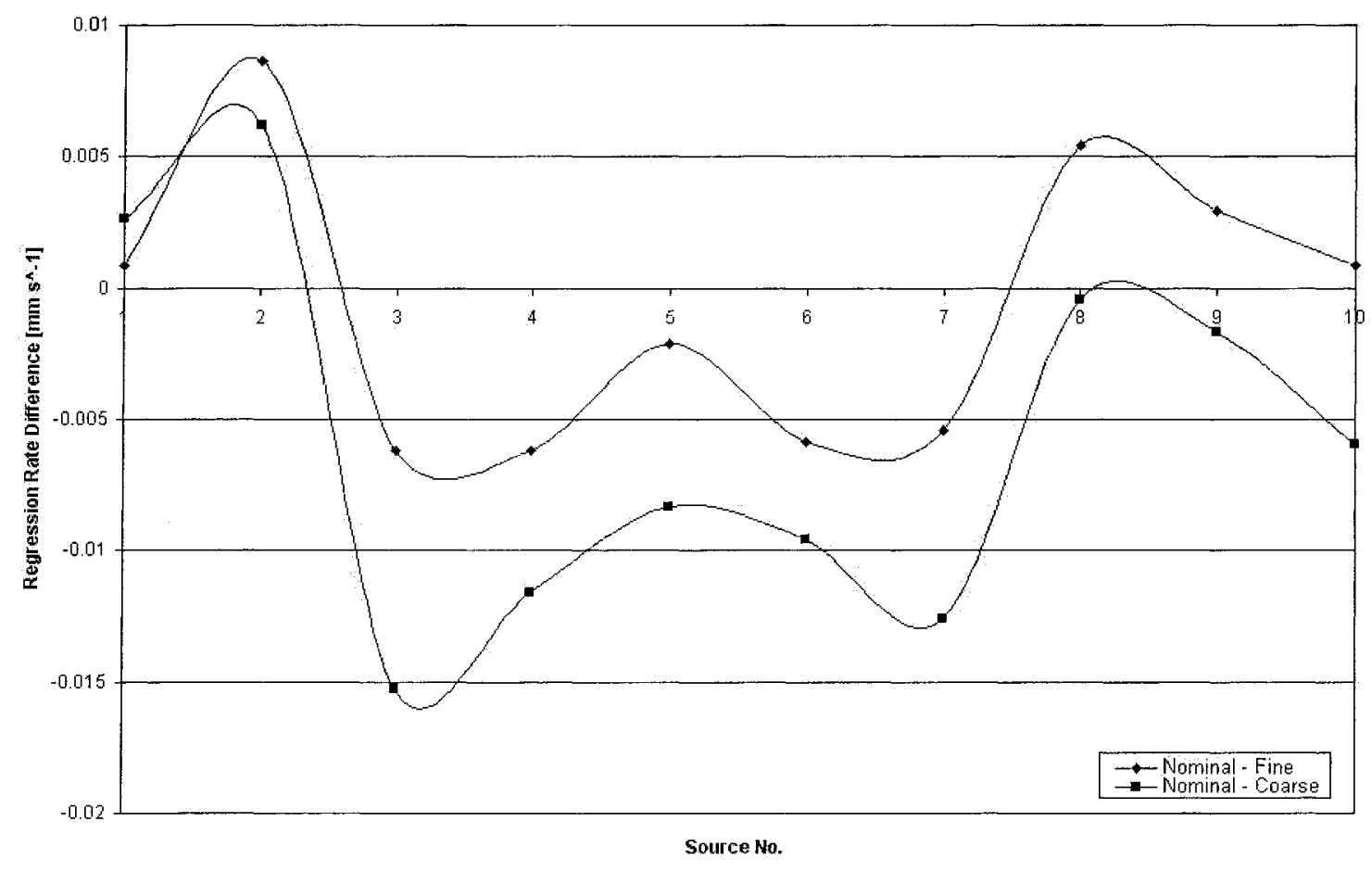

Figure 3.6: Change in regression rate with mesh refinement relative to nominal mesh. 


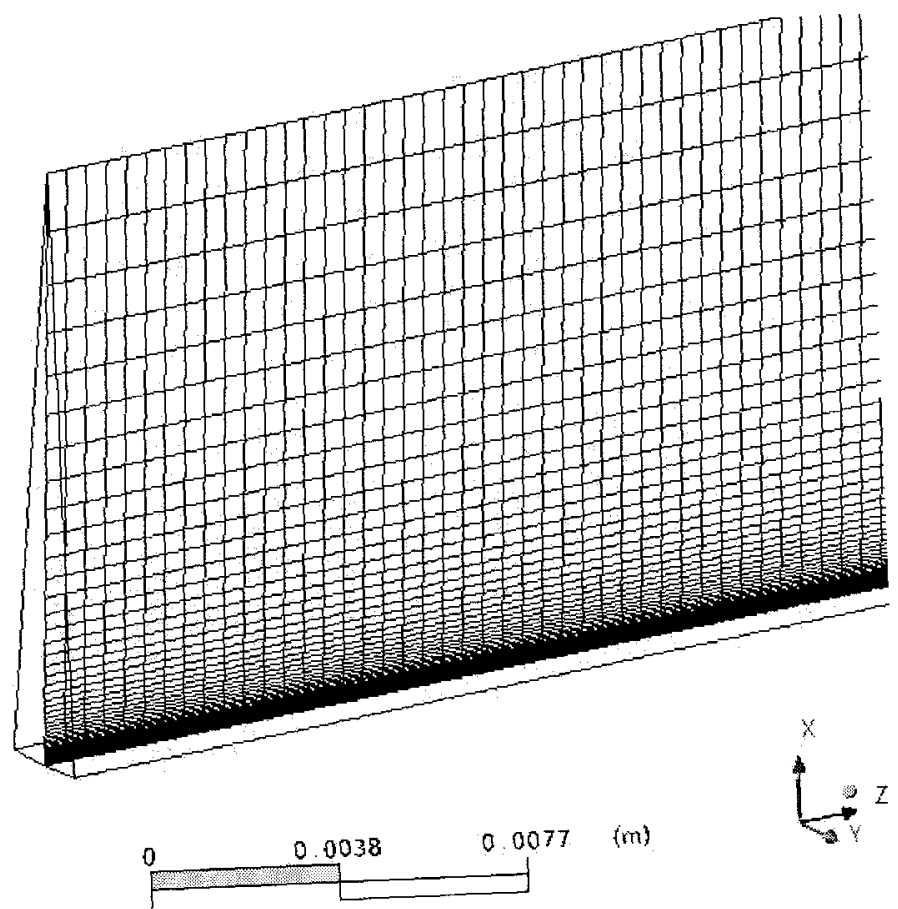
Figure 3.7: XZ plane showing mesh and coordinate axes for the HTPB14 model. 
Table 3.1: Mesh statistics used during refinement.

\begin{tabular}{|l|l|l|l|}
\hline Mesh & Nominal & Coarse & Fine \\
\hline \hline Radial Node Count & 71 & 51 & 91 \\
Axial Node Count & 811 & 811 & 811 \\
First Node Spacing & $0.001 \mathrm{~mm}$ & $0.001 \mathrm{~mm}$ & $0.001 \mathrm{~mm}$ \\
Total Node Count & 341431 & 244111 & 438751 \\
r parameter in Equation 3.18 & 1.11 & 1.17 & 1.08 \\
\hline
\end{tabular}

Table 3.2: Mesh statistics for HRM Model.

\begin{tabular}{|l|l|l|}
\hline Model & Test 14 (Model: HTPB14) & Test 15 (Model: HTPB15) \\
\hline \hline Radial Node Count & 71 & 96 \\
Axial Node Count & 811 & 811 \\
First Node Spacing & $0.001 \mathrm{~mm}$ & $0.001 \mathrm{~mm}$ \\
y+ & $0.4-0.9$ & $0.3-0.6$ \\
Total Node Count & 341431 & 463081 \\
\hline
\end{tabular}

\subsubsection{Boundary conditions and other model parameters}

The parameter values specified at the boundary conditions in both models are summarized in Table 3.3. No slip walls were specified at the fuel surfaces, with the wall temperature set to be the temperature of the mass injection in the sources. Symmetry boundary conditions were applied at the sides of the domain. The mass fractions of oxygen and water vapour at the inlet were based on those present in $90 \%$ by weight hydrogen peroxide, that was assumed to be fully decomposed upon exit from the catalyst pack. The temperature of the oxidizer at the inlet was set to $1022 \mathrm{~K}$, the adiabatic decomposition temperature of $90 \% \mathrm{HP}$ predicted by PROPEP. 
The turbulence intensity at the inlet was taken from measurements reported in [13] where it was found that the amplitude of the core flow turbulence at the inlet of the combustion chamber was approximately $10 \%$ of the mean velocity in the cold flow simulation tests. This result is obviously strongly injector dependent, and the value was used for lack of an experimental measurement. The fuel grain geometry corresponded to that used in Tests 14 and 15. The density for each test was calculated based on fuel grain geometry and fuel grain mass measurements. The reference pressure was set to the measured chamber pressure at the start of Tests 14 and 15 , after the initial start-up pressure transient.

Specifying the automatic turbulence length scale option at the inlet amounted to:

$$
\begin{gathered}
\varepsilon_{\text {inlet }}=\rho C_{\mu} \frac{k^{2}}{\mu_{t}} \\
k_{\text {inlet }}=\frac{3}{2} I^{2} U^{2} \\
\mu_{t}=1000 I \mu_{\text {inlet }}
\end{gathered}
$$

where $\mathrm{I}$ is the specified intensity, $\mathrm{k}$ the turbulence kinetic energy, and $\mu_{t}$ the eddy viscosity. 
Table 3.3: Model parameters and boundary conditions.

\begin{tabular}{|l|l|l|}
\hline Model & HTPB14 (Test 14) & HTPB15 (Test 15) \\
\hline \hline Geometry & & \\
\hline grain length & $391.5 \mathrm{~mm}$ & $391.5 \mathrm{~mm}$ \\
port diameter & $25.4 \mathrm{~mm}$ & $38.1 \mathrm{~mm}$ \\
\hline Reference Pressure & $588 \mathrm{psi}$ & $586 \mathrm{psi}$ \\
\hline Fuel Density & $907.1 \frac{\mathrm{kg}}{\mathrm{m}^{3}}$ & $906.8 \frac{\mathrm{kg}}{\mathrm{m}^{3}}$ \\
\hline Inlet & & \\
\hline mass flow & $0.00325 \frac{\mathrm{kg}}{\mathrm{s}}$ & $0.00344 \frac{\mathrm{kg}}{\mathrm{s}}$ \\
static temperature & $1022 \mathrm{~K}$ & $1022 \mathrm{~K}$ \\
mass fraction - oxygen & 0.423 & 0.423 \\
mass fraction - water & 0.577 & 0.577 \\
turbulence intensity & 0.1 & 0.1 \\
turbulence length scale & automatic (see Eqn. 3.19) & automatic \\
thermal radiation & local temperature & local temperature \\
\hline Outlet & & \\
\hline average static pressure & 0 psi & 0 psi \\
thermal radiation & local temperature & local temperature \\
\hline Fuel Walls & & \\
\hline velocity & no slip & no slip \\
energy & T & Ts \\
emissivity & 0.95 & 0.95 \\
diffuse fraction & 1.0 & 1.0 \\
\hline
\end{tabular}

\subsubsection{Solver control}

Solver control parameters that have not been discussed in previous subsections are included in Table 3.4 .

The conservative automatic time step calculated in CFX 5.7.1 was typically 0.00026 seconds and the same time step was used for all equations. This time step gave fairly smooth convergence without oscillations of the equations residuals for 
Table 3.4: Solver control parameters.

\begin{tabular}{|l|l|}
\hline Parameter & Value \\
\hline \hline Advection Term Discretization & Specified Blend Factor $=1.0$ (second order accurate) \\
Time Step & automatic, conservative \\
Residual Target & $0.0001 \mathrm{MAX}$ \\
Conservation Target & 0.01 \\
Precision & double \\
\hline
\end{tabular}

both models.

\subsection{HRM submodels}

\subsubsection{Material models}

The injected oxidizer was $90 \%$ by weight hydrogen peroxide which resulted in inlet values for the oxygen and water mass fractions of $Y_{\mathrm{O}_{2}}=0.423$ and $Y_{\mathrm{H}_{2} \mathrm{O}}=0.577$. The products of fuel pyrolysis were taken to be entirely 1,3-butadiene $\left(\mathrm{C}_{4} \mathrm{H}_{6}\right)$ following work by other researchers $[18],[20],[21]$. Experimental data by Chiaverini et al. [22] gave the relative molar concentration of the pyrolysis products of HTPB at various surface temperatures. The data indicated that the composition was primarily 1,3-butadiene, and that the portion of 1,3-butadiene in the products decreased with increasing pyrolysis temperature.

The equations that follow are found in Reference [39] unless otherwise stated. The ideal gas equation of state and constitutive relation used for the fluid 
mixture are given by Equations 3.22 and 3.23.

$$
\begin{gathered}
\overline{\rho_{\text {mix }}}=\frac{w_{\operatorname{mix}}\left(p+p_{\text {ref }}\right)}{R u T} \\
\widetilde{h_{\text {stat }}}-h_{\text {ref }}=\int_{T_{\text {ref }}}^{T_{\text {stat }}} C_{p}(T) d T
\end{gathered}
$$

The total enthalpy is given by:

$$
\widetilde{h_{\text {tot }}}=\widetilde{h_{\text {stat }}}+\frac{1}{2} \tilde{u_{i}} \tilde{u_{i}}+k
$$

The multicomponent fluid properties used in the preceding equations are evaluated in the following manner.

For mixture molar mass [50]:

$$
\frac{1}{w_{m i x}}=\sum_{i=A, B \ldots}^{N_{C}} \frac{\tilde{Y}_{i}}{w_{i}}
$$

For other mixture properties $\left(\mu, \lambda, \widetilde{h_{\text {stat }}}\right)$ :

$$
\alpha=\sum_{i=A, B \ldots}^{N_{C}} \tilde{Y}_{i} \alpha_{i}
$$

where $\alpha$ is the material property in question.

The specific heat capacity, reference enthalpy, viscosity, and thermal conduc- 
tivity for oxygen $\left(\mathrm{O}_{2}\right)$, carbon monoxide $(\mathrm{CO})$, carbon dioxide $\left(\mathrm{CO}_{2}\right)$, water vapour $\left(\mathrm{H}_{2} \mathrm{O}\right)$, and hydrogen $\left(\mathrm{H}_{2}\right)$ were evaluated as a function of temperature using empirically determined polynomials from [51] for a temperature range of $300 \mathrm{~K} \leq \mathrm{T}$ $\leq 5000 \mathrm{~K}$. The specific heat capacity and reference enthalpy for 1,3-butadiene was also taken from this reference. The transport properties $(\mu, \lambda)$ of 1,3 -butadiene were taken from [52] for a temperature range of $300 \mathrm{~K} \leq \mathrm{T} \leq 1300 \mathrm{~K}$. The diffusion coefficients of the constituents were assumed to be equal to the molecular viscosity, an approximation that was deemed valid due to the much larger effect of turbulence fluctuations than molecular diffusion on species mixing.

\subsubsection{Turbulence model}

The terms requiring turbulence modeling in order to close the set of governing equations were identified in Equations 3.2, 3.3, and 3.4.

Terms I \& III: Turbulent scalar flux was modeled using the gradientdiffusion hypothesis.

$$
\begin{gathered}
\overline{\rho Y_{i}^{\prime \prime} u_{j}^{\prime \prime}}=\frac{-\mu_{t}}{S c_{t}} \frac{\partial \tilde{Y}_{i}}{\partial x_{j}} \\
\overline{\rho h^{\prime \prime} u_{j}^{\prime \prime}}=\frac{-\mu_{t}}{P r_{t}} \frac{\partial \tilde{h}}{\partial x_{j}}
\end{gathered}
$$

Term II: A linear relationship between Reynolds stress and rate of strain 
was used to model the Reynolds stress tensor.

$$
\overline{\rho u_{i}^{\prime \prime} u_{j}^{\prime \prime}}=\underbrace{\frac{2}{3} \bar{\rho} k \delta_{i j}}_{\mathbf{I X}}+\underbrace{\frac{2}{3} \mu_{t} \frac{\partial \tilde{u_{k}}}{\partial x_{k}} \delta_{i j}}_{\mathbf{X}}-\mu_{t}\left(\frac{\partial \tilde{u_{i}}}{\partial x_{j}}+\frac{\partial \tilde{u_{j}}}{\partial x_{i}}\right)
$$

The following terms were neglected in the form of the governing equations implemented in CFX 5.7 .1 [43]:

1. Term IX in the Reynolds stress tensor;

2. Term $\mathrm{X}$ in the Reynolds stress tensor.

The apparent normal stress due to turbulence kinetic energy is small in flows where $\bar{\rho} k<<P$ as in this study, and can be safely neglected[44]. Term X involving the dilatation term is neglected for the same reasons stated in Section 3.3.1.

The turbulent eddy viscosity $\left(\mu_{t}\right)$ was obtained using the Shear Stress Transport model (SST) through the solution of a weighted combination of the standard $\mathrm{k}-\omega$ model in the near-wall region, and the standard $\mathrm{k}-\varepsilon$ model in the main flow region. The SST model was chosen because the formulation in CFX allows one to solve the equations on a mesh that has the first node in the viscous sublayer of the boundary layer, thus resolving the remainder of the boundary layer in the solution without the use of wall functions.

The form of the equations for the implementation of these turbulence models in CFX 5.7.1 is given below for completeness. The constants are defined in Table 
3.5. Refer to Reference [40] for details regarding the particular formulation of the blending and limiter functions used in the SST model.

$$
\frac{\partial(\bar{\rho} k)}{\partial t}+\frac{\partial}{\partial x_{j}}\left(\bar{\rho} \tilde{u}_{j} k\right)=\frac{\partial}{\partial x_{j}}\left[\left(\mu+\frac{\mu_{t}}{\sigma_{k 1}}\right) \frac{\partial k}{\partial x_{j}}\right]-\underbrace{\overline{\rho u_{i}^{\prime \prime} u_{j}^{\prime \prime}} \frac{\partial \tilde{u}_{i}}{\partial x_{j}}}_{\text {turbulence production }}-\frac{\mu_{t}}{\bar{\rho}^{2}} \frac{\partial \bar{\rho}}{\partial x_{i}} \frac{\partial \bar{p}}{\partial x_{i}}-\bar{\rho} \varepsilon
$$

$$
\frac{\partial(\bar{\rho} \varepsilon)}{\partial t}+\frac{\partial}{\partial x_{j}}\left(\bar{\rho} \tilde{u}_{j} \varepsilon\right)=\frac{\partial}{\partial x_{j}}\left[\left(\mu+\frac{\mu_{t}}{\sigma_{\varepsilon}}\right) \frac{\partial \varepsilon}{\partial x_{j}}\right]-C_{\varepsilon 1} \frac{\varepsilon}{k}\left(\overline{\rho u_{i}^{\prime \prime} u_{j}^{\prime \prime}} \frac{\partial \tilde{u}_{i}}{\partial x_{j}}+\frac{\mu_{t}}{\bar{\rho}^{2}} \frac{\partial \bar{\rho}}{\partial x_{i}} \frac{\partial \bar{p}}{\partial x_{i}}\right)-C_{\varepsilon 2} \bar{\rho} \frac{\varepsilon^{2}}{k}
$$

$$
\frac{\partial(\bar{\rho} k)}{\partial t}+\frac{\partial}{\partial x_{j}}\left(\bar{\rho} \tilde{u_{j}} k\right)=\frac{\partial}{\partial x_{j}}\left[\left(\mu+\frac{\mu_{t}}{\sigma_{k 2}}\right) \frac{\partial k}{\partial x_{j}}\right]-\overline{\rho u_{i}^{\prime \prime} u_{j}^{\prime \prime}} \frac{\partial \tilde{u}_{i}}{\partial x_{j}}-\frac{\mu_{t}}{\bar{\rho}^{2}} \frac{\partial \bar{\rho}}{\partial x_{i}} \frac{\partial \bar{p}}{\partial x_{i}}-\beta^{\prime} \bar{\rho} k \omega
$$

$$
\frac{\partial(\bar{\rho} \omega)}{\partial t}+\frac{\partial}{\partial x_{j}}\left(\bar{\rho} \tilde{u_{j}} \omega\right)=\frac{\partial}{\partial x_{j}}\left[\left(\mu+\frac{\mu_{t}}{\sigma_{\omega}}\right) \frac{\partial \omega}{\partial x_{j}}\right]-\alpha \frac{\omega}{k}\left(\overline{\rho u_{i}^{\prime \prime} u_{j}^{\prime \prime}} \frac{\partial \tilde{u}_{i}}{\partial x_{j}}+\frac{\mu_{t}}{\bar{\rho}^{2}} \frac{\partial \bar{\rho}}{\partial x_{i}} \frac{\partial \bar{p}}{\partial x_{i}}\right)-\beta \bar{\rho} \omega^{2}
$$

Note that the term involving $\left(\frac{\partial \bar{\rho}}{\partial x_{i}} \frac{\partial \bar{p}}{\partial x_{i}}\right)$ is neglected in the form of the equations used in CFX 5.7.1, a simplification that is strictly only valid for constant density flows, but is commonly made in practice. Discussion of this point is found 
in Kuo[41]. The form of the Reynolds stress tensor used in the turbulence production terms of Equations 3.30, 3.31, 3.32 and 3.33 included the dilatation term, modified by a constant to avoid the build-up of turbulence kinetic energy and dissipation across shocks.

$$
\overline{\rho u_{i}^{\prime \prime} u_{j}^{\prime \prime}}=\frac{2}{3}\left(\bar{\rho} k \delta_{i j}+3 \mu_{t} \frac{\partial \tilde{u_{k}}}{\partial x_{k}} \delta_{i j}\right)-\mu_{t}\left(\frac{\partial \tilde{u_{i}}}{\partial x_{j}}+\frac{\partial \tilde{u_{j}}}{\partial x_{i}}\right)
$$

In the $\mathrm{k}-\varepsilon$ model, the eddy viscosity is given as:

$$
\mu_{t}=C_{\mu} \bar{\rho} \frac{k^{2}}{\varepsilon}
$$

In the $k-\omega$ model, the eddy viscosity is given as:

$$
\mu_{t}=\bar{\rho} \frac{k}{\omega}
$$

The eddy viscosity in the SST model is a weighted sum of these two quantities.

To determine the fluctuating turbulent transport properties $\lambda_{t}, C p_{t}$ and $D_{t}$, the turbulent Lewis number is assumed equal to one, and the turbulent Prandt? number set equal to 0.9 such that: 
Table 3.5: Constants for the SST turbulence model.

\begin{tabular}{|l|l|}
\hline Constant & Value \\
\hline \hline$C_{\varepsilon 1}$ & 1.44 \\
$C_{\varepsilon 2}$ & 1.92 \\
$C_{\mu}$ & 0.09 \\
$\sigma_{k 1}$ & 1.0 \\
$\sigma_{k 2}$ & 2.0 \\
$\sigma_{\varepsilon}$ & 1.3 \\
$\sigma_{\omega}$ & 2.0 \\
$\beta$ & 0.075 \\
$\beta^{\prime}$ & 0.09 \\
$\alpha$ & $\frac{5}{9}$ \\
\hline
\end{tabular}

$$
\begin{array}{r}
P r_{t}=\frac{\mu_{t} C p_{t}}{\lambda_{t}}=0.9 \\
S c_{t}=\frac{\mu_{t}}{\rho D_{t}} \\
L e_{t}=\frac{S c_{t}}{P r_{t}}=1.0
\end{array}
$$

At the first node from the wall the shear stress in the momentum equation is given by the wall function formulation discussed in 3.3.3. The turbulence kinetic energy flux is set to zero at this node, and the value of $\omega$ is given by Equations 3.38 [40]. In the HRM models the value for $\omega$ in the sublayer, $\omega_{s}$, dominated due to the small mesh spacing at the wall. 


$$
\begin{array}{r}
\omega_{l}=\frac{1}{a_{1} \kappa \nu} \frac{u^{*}}{y^{*}} \\
\omega_{s}=\frac{6 \nu}{\beta(\Delta y)^{2}} \\
\omega_{w}=\omega_{s} \sqrt{1+\left(\frac{\omega_{l}}{\omega_{s}}\right)}
\end{array}
$$

\subsubsection{Combustion model}

The combustion mechanism of 1,3-butadiene with hydrogen peroxide was approximated by a simplified two step reaction model [20], given by Equations 3.41 and 3.42 .

$$
\mathrm{C}_{4} \mathrm{H}_{6}+3 \mathrm{O}_{2} \rightarrow 4 \mathrm{CO}+2 \mathrm{H}_{2} \mathrm{O}+\mathrm{H}_{2}
$$

$$
\mathrm{CO}+0.5 \mathrm{O}_{2} \rightarrow \mathrm{CO}_{2}
$$

The effects of combustion on the flow field are felt via the source term $\left(S_{i}\right)$ in Equation 3.2. The form of this term for fluid component i, with $k=1 \ldots K$ elementary reactions was as follows.

$$
S_{i}=w_{i} \sum_{k=1}^{K}\left(\nu_{k i}^{\prime \prime}-\nu_{k i}^{\prime}\right) R_{k}
$$


Here $\nu_{k i}$ was the stoichiometric coefficient for species $\mathrm{i}$ in reaction $\mathrm{k}$, a superscript " denotes products and 'denotes reactants. The rate of progress for reaction $\mathrm{k}, R_{k}$, was modeled using the combined Eddy Dissipation Finite Rate combustion model in CFX 5.7.1. This model computed both the Eddy Dissipation reaction rate and the Finite Rate reaction rate and used the lesser of the two for the particular node location.

The Finite Rate or Arrhenius form for the forward reaction rate of the first global reaction, 3.41, was taken from [20].

$$
F_{1 F R}=8.8 \cdot 10^{11} \frac{\mathrm{cm}^{2.25}}{\mathrm{~s} \cdot \mathrm{mol} l^{0.75}} e^{-\frac{1.52 \cdot 10^{4}}{T}}
$$

The rate of progress for this global reaction was then [54]:

$$
R_{1 F R}=F_{1 F R}\left[C_{4} H_{6}\right]^{0.15}\left[O_{2}\right]^{1.6}
$$

The reaction rate given in [53] for the CO Oxidization reaction step had a functional dependency on $\mathrm{H}_{2} \mathrm{O}$ and therefore could not be used in this study due to the abundance of water vapour in the oxidizer. The reaction rate for the $\mathrm{CO}$ Oxidization step was therefore determined solely via the Eddy Dissipation Combustion Model. 
The form of the rate of progress variable given by the Eddy Dissipation Combustion Model was as follows.

$$
R_{k E D C}=A_{E D M} \frac{\varepsilon}{k}\left(\min \frac{[i]}{\nu_{k i}^{\prime}}\right)
$$

Where $\left(A_{E D M}=4\right)$ was a model constant as defined by Magnussen[55], and $\varepsilon$ and $k$ were determined from the solution of the turbulence transport equations. The Eddy Dissipation Combustion Model is based on the assumption of fast chemistry (large Damköhler number), and it was used in this study under the assumption that the rate of combustion in turbulent diffusion flames is determined by the rate of mixing of the fuel and oxidizer on the molecular scale; the rate of dissipation of the eddies. The value of the constant $A_{E D M}$ is based on consideration of a turbulence energy cascade model that makes a connection between the viscous fine structures and the larger, energy transporting eddies that are simulated by the turbulence transport models. This cascade model was compared to measured turbulence energy spectra in the inertial subrange and dissipative range for reacting and non-reacting flows by Ertesvag et al.[56] with good agreement. The model has been applied to turbulent diffusion flames (for example, in Reference [57]) and turbulent premixed flames (for example, in Reference [55]) with success. 


\subsubsection{Radiation model}

The spectral radiative transfer equation (RTE) to be solved in CFX 5.7.1 had the following form [58].

$\frac{d I_{\nu}(\mathbf{r}, \mathbf{s})}{d s}=-\left(K_{a \nu}+K_{s \nu}\right) I_{\nu}(\mathbf{r}, \mathbf{s})+K_{a} I_{b}(\nu, T)+\frac{K_{s \nu}}{4 \pi} \int_{4 \pi} d I_{\nu}\left(\mathbf{r}, \mathbf{s}^{\prime}\right) \Phi\left(\mathbf{s} \bullet \mathbf{s}^{\prime}\right) d \Omega^{\prime}+S$

where

$\nu=$ frequency

$\mathbf{r}=$ position vector

$\mathrm{s}=$ direction vector

$\mathrm{s}=$ path length

$K_{a}=$ absorption coefficient

$K_{s}=$ scattering coefficient

$I_{b}=$ blackbody emission intensity

$I_{\nu}=$ spectral radiation intensity which depends on position and direction

$\mathrm{T}=$ local absolute temperature

$\Omega=$ solid angle

$\Phi=$ in phase scattering function

$\mathrm{S}=$ radiation source intensity term

The boundary conditions for $I_{\nu}$ were given as follows. The solid fuel was defined as a glossy, opaque rubber (due to the carbon black content) with an emissivity of $0.95[23]$ and a diffuse fraction of 1.0. Both the inlet and the outlet were 
specified as blackbodies radiating at the local fluid temperature. In the case of the outlet boundary this was likely an underestimation of the radiative heat transfer into the domain as the black phenolic/graphite nozzle would have been a source of radiation and may have been at a higher temperature than the mean temperature of the fluid exiting the combustion chamber.

The solution of Equation 3.47 was approximated using the P-1 Radiative Transfer Model in CFX 5.7.1, also known as the Differential Approximation. The assumption of the model was that the radiation intensity was isotropic in space.

The spectral dependency of the radiation intensity field was modeled using the Multigray spectral model in CFX 5.7.1. Values for relative absorption and emission from combustion gases were taken to be functions of the partial pressure of the constituents, the temperature, and the path length. The constituents accounted for in the model were $\mathrm{C}_{4} \mathrm{H}_{6}, \mathrm{CO}_{2}, \mathrm{CO}, \mathrm{H}_{2} \mathrm{O}$.

The emissivity was assumed to have the following functional dependence:

$$
\epsilon_{g}=\sum_{i=1}^{N_{g}} a_{g i}\left(1-e^{-K_{i} p_{i} L}\right)
$$

where

$\epsilon_{g}$ is the total emissivity of the gas mixture

$N_{g}$ is the number of grey gases

$p_{i} L$ is the product of the partial pressure and path length of gas i 
$a_{g i}$ is the grey gas $\mathrm{i}$ weight or amplitude

$K_{i}$ is the monochromatic absorption coefficient for gas i [59].

The parameters in this model were defined using the formulae presented in the CFX Radiation Theory Manual [58] which was taken from the work by Leckner[19]. This spectral model was also used in the numerical models presented in References [18] and [23]. The coefficients for the model are summarized in Table 3.6 .

$$
\begin{array}{r}
a_{g i}=b_{1 i}+10^{-5} b_{2 i} T_{g} \\
K_{i} \rightarrow K_{i}\left(p_{H_{2} \mathrm{O}}+p_{C \mathrm{O}_{2}}+p_{C O}\right)+K_{H C i} p_{C_{4} H_{6}}
\end{array}
$$

Table 3.6: Summary of coefficients for the grey gas spectral model.

\begin{tabular}{|l|l|l|l|l|}
\hline $\mathrm{i}$ & $b_{1 i}$ & $b_{2 i}\left[K^{-1}\right]$ & $K_{i}\left[m^{-1} a_{t m}^{-1}\right]$ & $K_{H C i}\left[m^{-1} a t m^{-1}\right]$ \\
\hline \hline 1 & 0.364 & 4.74 & 0 & 3.85 \\
2 & 0.266 & 7.19 & 0.69 & 0 \\
3 & 0.252 & -7.41 & 7.4 & 0 \\
4 & 0.118 & -4.52 & 80 & 0 \\
\hline
\end{tabular}

Soot formation and therefore radiation from soot was neglected in the present model. It should be noted that in experiments with HTPB/GOX (gaseous oxygen) [23], the radiative contribution from soot was found to contribute approximately $25 \%$ of the total radiant heat flux measured at low mass flux $\left(\sim 100 \frac{\mathrm{kg}}{\mathrm{m}^{2} \mathrm{~s}}\right)$ and fuel 
rich conditions.

\subsubsection{Fuel ablation model}

To examine the variation of fuel surface temperature and regression rate along the length of the fuel grain, the fuel wall was divided into ten evenly sized sources, and the mass flow and temperature for the injected fluid was calculated individually for each using source using a FORTRAN subroutine that is described in the next subsection. The geometry and mesh of the last source on the model is shown in Figure 3.8. The ablation of the fuel surface was assumed to follow an Arrhenius type law as given in Equation 3.51; any heterogeneous surface reactions were neglected. The activation energy and preexponential factor for HTPB was taken from Reference [22] and were valid for fuel surface temperatures greater than $722 \mathrm{~K}$.

$$
\dot{r}=A r \cdot e^{-\frac{E a}{R u \cdot T_{s}}}
$$

Where $E a=4.91 \frac{\mathrm{kcal}}{\mathrm{mol}}, A r=11.04 \frac{\mathrm{mm}}{\mathrm{s}}$.

The energy balance at the fuel surface was given by Equation 3.52. The wall heat flux, $q_{w}$, in this equation was taken as the area averaged value, and output for each of the ten regions of the fuel wall from the CFX solver. It included both the radiative and convective heat flux at the fuel wall. The energy of pyrolysis and specific heat capacity at constant pressure for HTPB were taken from Reference [35]. 


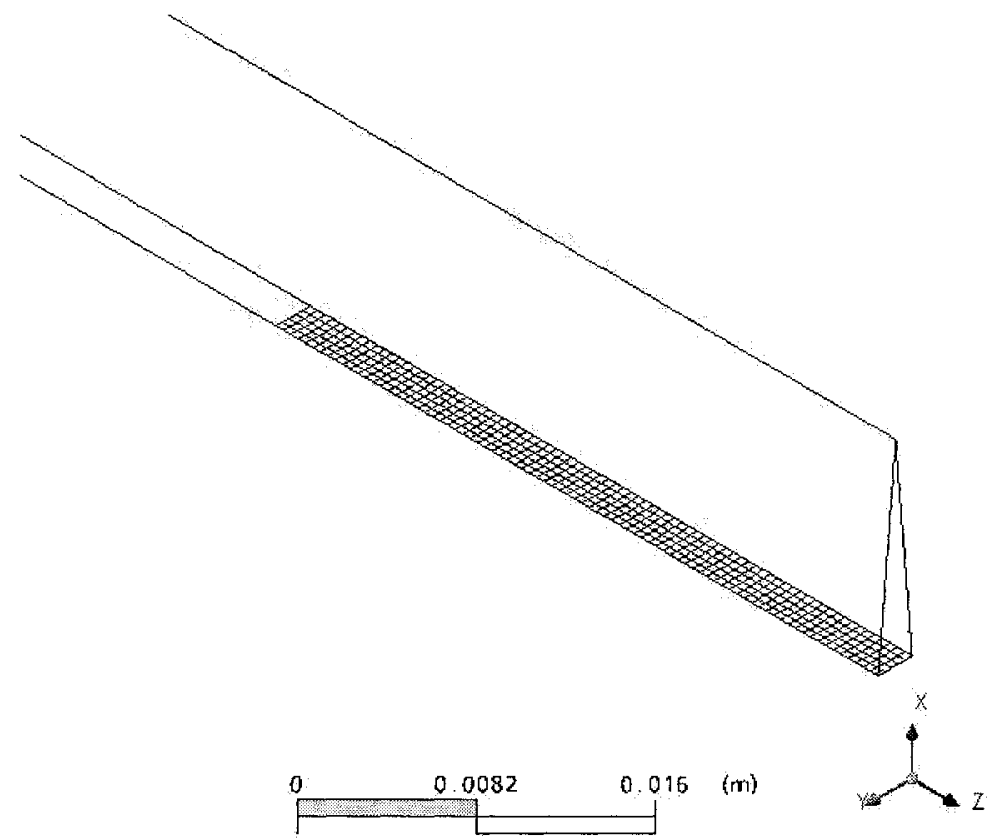

Figure 3.8: View of source number ten in the HTPB14 model. Source was $0.001 \mathrm{~mm}$ thick.

The fuel density was determined experimentally. The conductive heat flux through the fuel grain was calculated assuming an exterior fuel grain temperature equal to the ambient temperature, which in this case was also equal to the initial fuel grain temperature. This approximation is valid for the initial conditions of the motor burn. It is also a good approximation for a quickly regressing fuel surface as the subsurface thermal boundary is thin in that case [60]. The form of the expression for the heat flux conducted through the fuel was obtained from [61].

$$
q_{w}=\rho_{f} \dot{r}\left(h_{d}+C p_{f}\left(T_{s}-T_{r e f}\right)\right)+\rho_{f} \dot{r} C p_{f}\left(T_{s}-T_{a m b .}\right)
$$


Where $h_{d}=430 \frac{\mathrm{cal}}{g}, T_{\text {ref }}=T_{a m b}=298 \mathrm{~K}$ and $C p_{f}=0.39 \frac{\mathrm{cal}}{\mathrm{gK}}$.

The values for the variables that were specified at each subdomain source are summarized in Table 3.7. The velocity of the injected mass at the source was calculated by the CFX solver with the specification of mass injection temperature and mass flux, as the fluid injection pressure was obtained from the interior of the domain. Because the model was an approximation of a situation where the fuel wall is actually regressing at a speed equal to the fuel regression rate, this injection velocity was not the absolute velocity but rather the velocity relative to the regressing fuel surface. The difference between the two velocities was $<0.001$ $\frac{m}{s}$ and was considered negligible for the purpose of this study.

Table 3.7: Summary of quantities specified at each subdomain source.

\begin{tabular}{|l|l|}
\hline Variable & Value \\
\hline \hline total mass injection & $\rho_{f} \dot{r} S A_{f}$ \\
injection temperature & $T_{s}$ \\
mass fraction $-C_{4} H_{6}$ & 1.0 \\
turbulence kinetic energy & $0 \frac{m^{2}}{s^{2}}$ \\
turbulence eddy dissipation & $0 \frac{m^{2}}{s^{3}}$ \\
cartesian velocity component - x & $\left|\frac{x}{\sqrt{x^{2}+y^{2}}}\right|$ \\
cartesian velocity component $-\mathrm{y}$ & $\frac{-y}{\sqrt{x^{2}+y^{2}}}$ \\
\hline
\end{tabular}

\subsubsection{CFX/FORTRAN interface}

The FORTRAN subroutine was written at Carleton specifically to interface with CFX 5.7.1. It received the area averaged wall heat flux for each of the ten sources 
from the converged CFX solution, calculated the temperature of the fuel surface and mass flow rate, and then passed these new values back to the CFX solver upon restart. The solution method was very similar to that presented by Serin and Gogus [18]. The whole procedure was iterated autonomously with the use of DOS batch files and took approximately 100 hours per model on a P4, $3.2 \mathrm{GHz}$ machine with 2 GB RAM.

The energy balance at the fuel surface, Equation 3.52, was solved iteratively in the code using temperature increments of $0.1 \mathrm{~K}$. The iteration between the FORTRAN code and the CFX solver was carried out until the change in temperature between subsequent solutions was less than $1.0 \mathrm{~K}$. A temperature change of $1.0 \mathrm{~K}$ produced a change in regression rate of approximately $0.002 \mathrm{~mm} / \mathrm{s}$, which was an order of magnitude less than the experimental error on the regression rate shown in Table 2.5 .

\subsection{Results}

The following section provides a summary of the results obtained from both numerical models, HTPB14 and HTPB15.

The fundamental quantitative measure of the predictive capability of the model was the comparison of the predicted spatially averaged regression rate to that measured experimentally in Tests 14 and 15. Additionally, the flame temperature 
was compared to the theoretical value for each test, and the location of the flame was compared to predictions from Venkateswaran and Merkle[21].

The temperature, density, and velocity profiles are presented. The shape of the temperature and velocity profiles in the near wall region are compared to those measured in combusting boundary layers with mass injection. The relative contributions of radiation and convection to the wall heat flux are shown for each model.

\subsubsection{Velocity, density and temperature fields}

Contour plots of the axial component of velocity, temperature and density fields obtained for the HTPB14 model are presented in Figures 3.9, 3.10 and 3.11. As the results for HTPB15 were qualitatively similar they are omitted here. Note that the combusting boundary layer does not reach the chamber centerline, indicating unreacted oxidizer entered the reaction chamber at the end of the fuel grain. For a motor with similar operating conditions as those in Tests 14 and 15, work by F.A. Williams indicated a length of approximately fifty port diameters was required for the boundary layer to completely fill the port and obtain fully developed flow [35], so this result is reasonable. The interesting characteristics of the near wall variable profiles are shown in subsequent sections. 


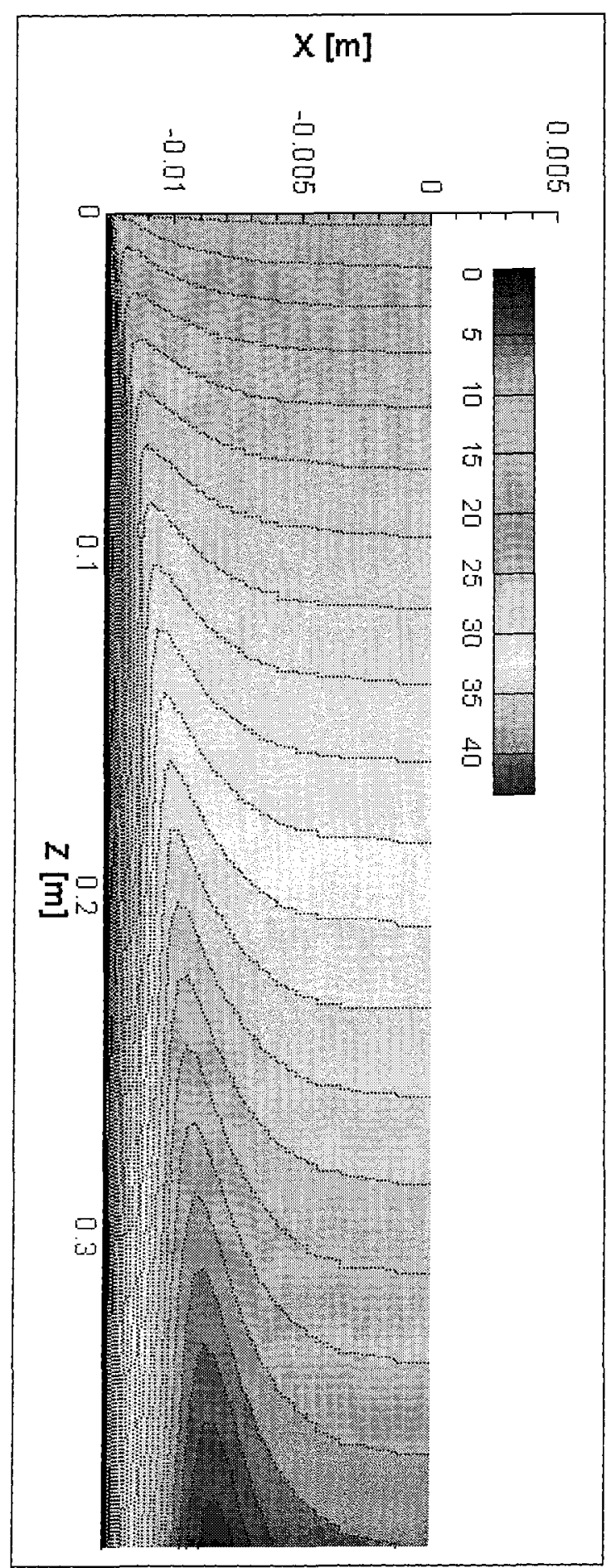

Figure 3.9: HTPB14 - Contours of the axial velocity component $[\mathrm{m} / \mathrm{s}]$. 


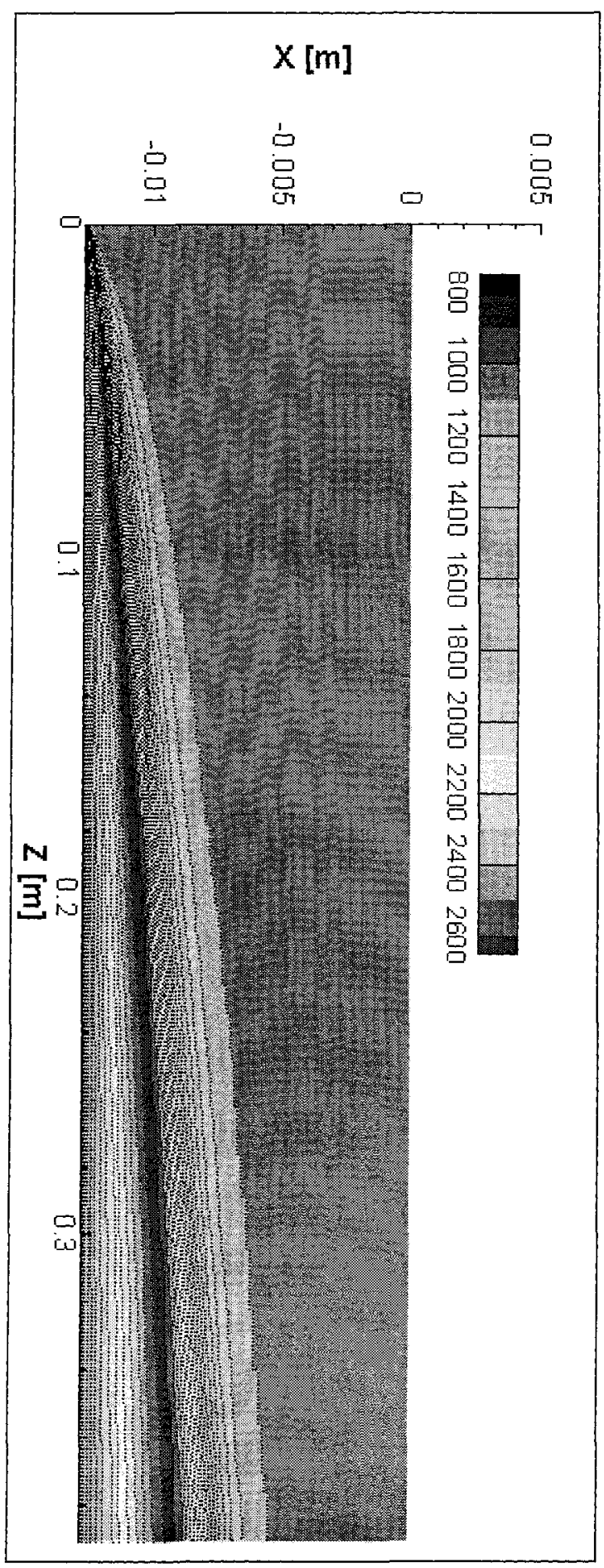

Figure 3.10: HTPB14 - Temperature contours [K]. 


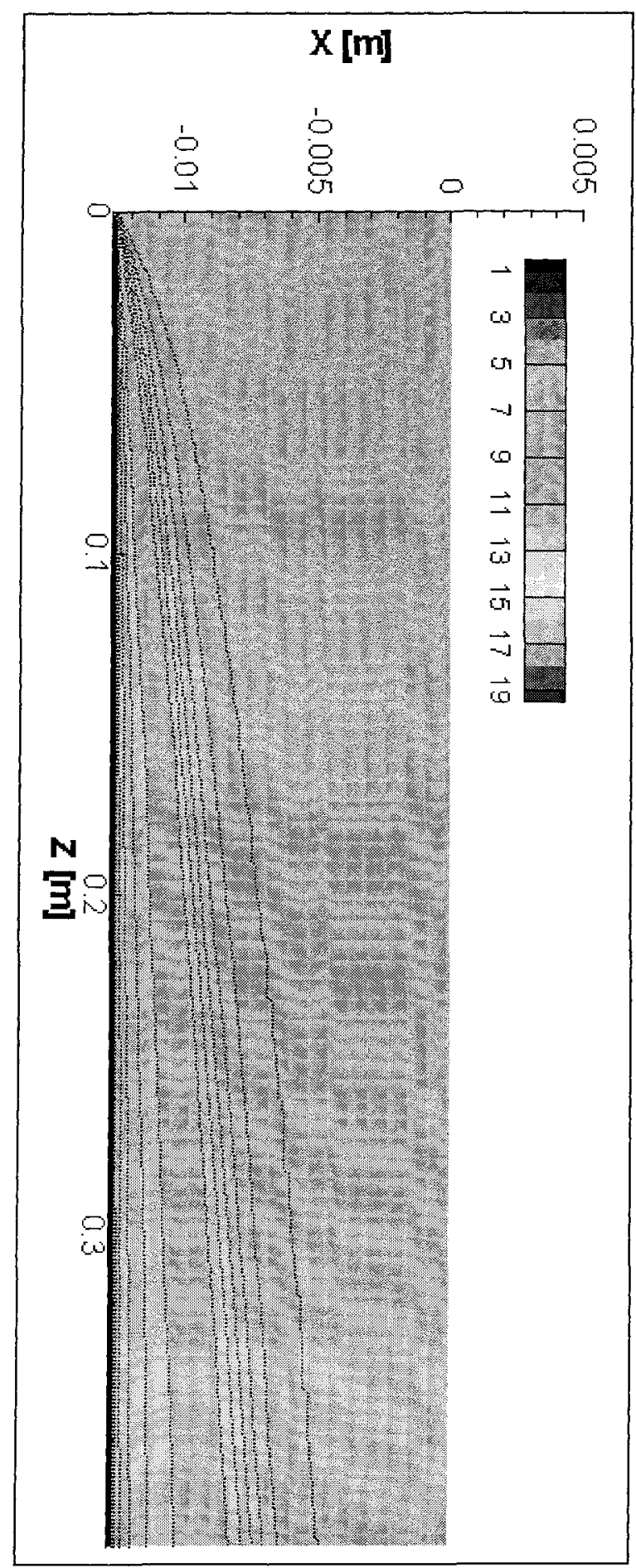

Figure 3.11: HTPB14 - Density contours $\left[\mathrm{kg} / \mathrm{m}^{3}\right]$. 


\subsubsection{Contributions to wall heat flux}

Figures 3.12 and 3.13 show the relative contributions of radiative and convective heat flux to the total heat flux calculated at the wall. The discontinuities in these figures indicate the source boundaries. The spike in the convective heat flux at the start of the port was due to the close proximity of the flame to the fuel surface at the start of the fuel grain. After this initial anomaly, the convective portion of the wall heat flux was approximately constant, while the radiative portion increased due to the increasing $\mathrm{CO}_{2}$ content in the combustion products. $\mathrm{CO}_{2}$ and $\mathrm{H}_{2} \mathrm{O}$ emissivity dominated the gas emissivity of the products, and the relative concentration of $\mathrm{H}_{2} \mathrm{O}$ in the HyperG experiments was high due to the composition of the decomposed HP.

Figure 3.14 illustrates the dependence of the convective heat flux on the motor port size and mass flux. At lower mass flux levels and larger port diameters, the radiative heat flux plays a larger role in the total wall heat flux of the motor as the radiative path length is longer, and the velocity gradient at the fuel surface more shallow. These effects have been produced in other numerical studies, for example that by Venkateswaran and Merkle[21] and also in experimental measurements by Chiaverini et al.[23]. 


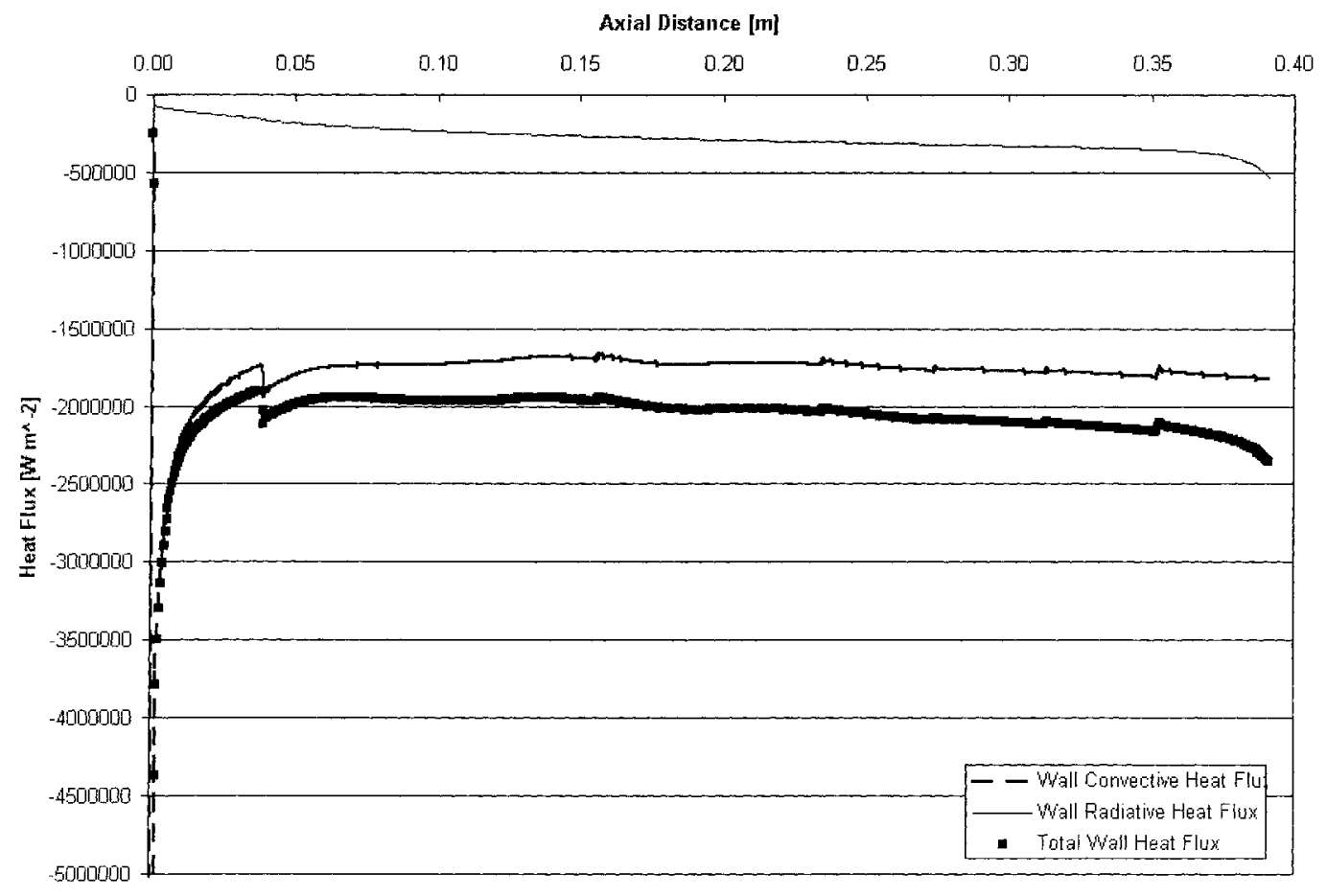

Figure 3.12: HTPB14 - Variation of wall heat flux in motor axial direction. 


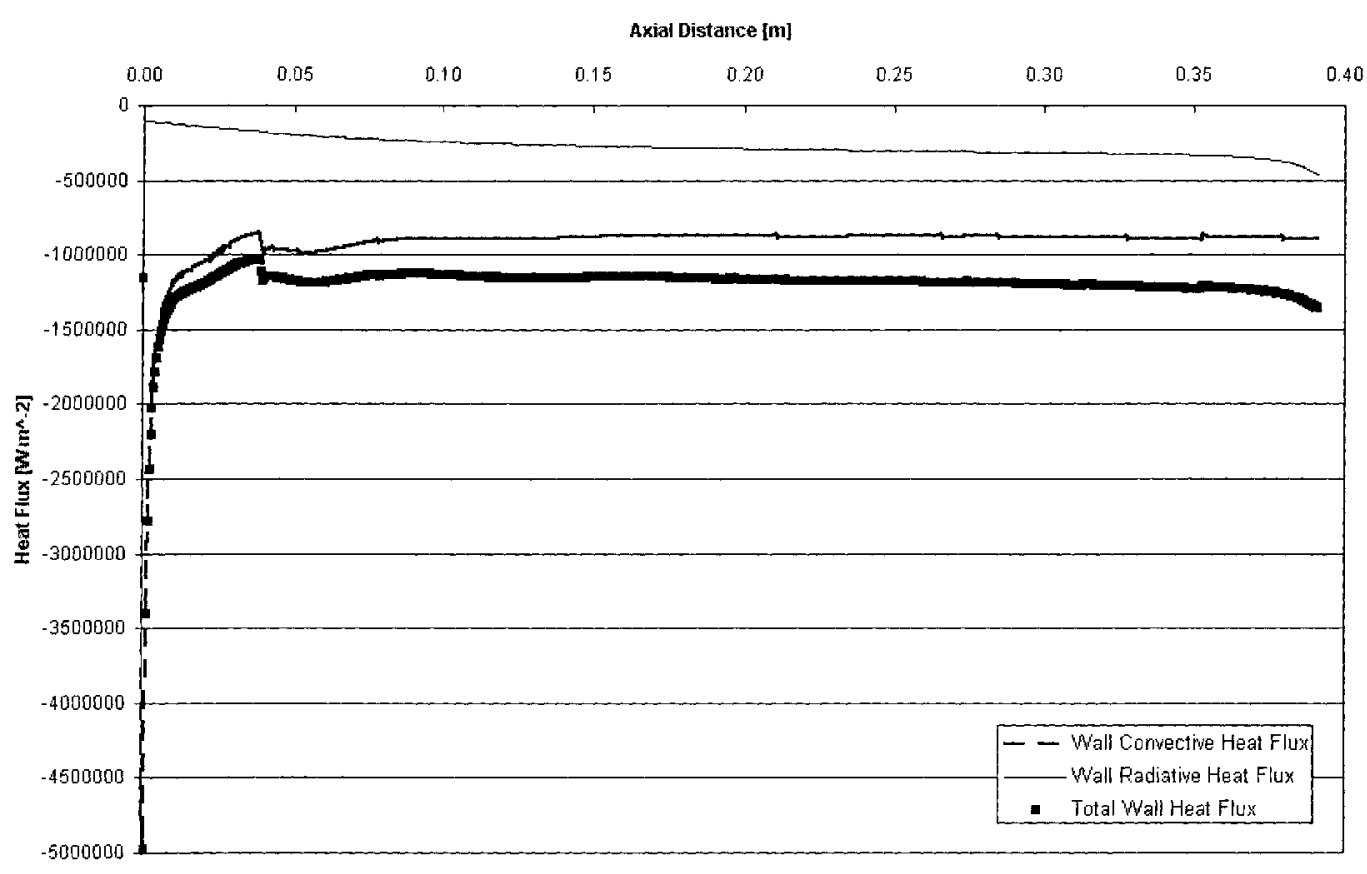

Figure 3.13: HTPB15 - Variation of wall heat flux in motor axial direction. 


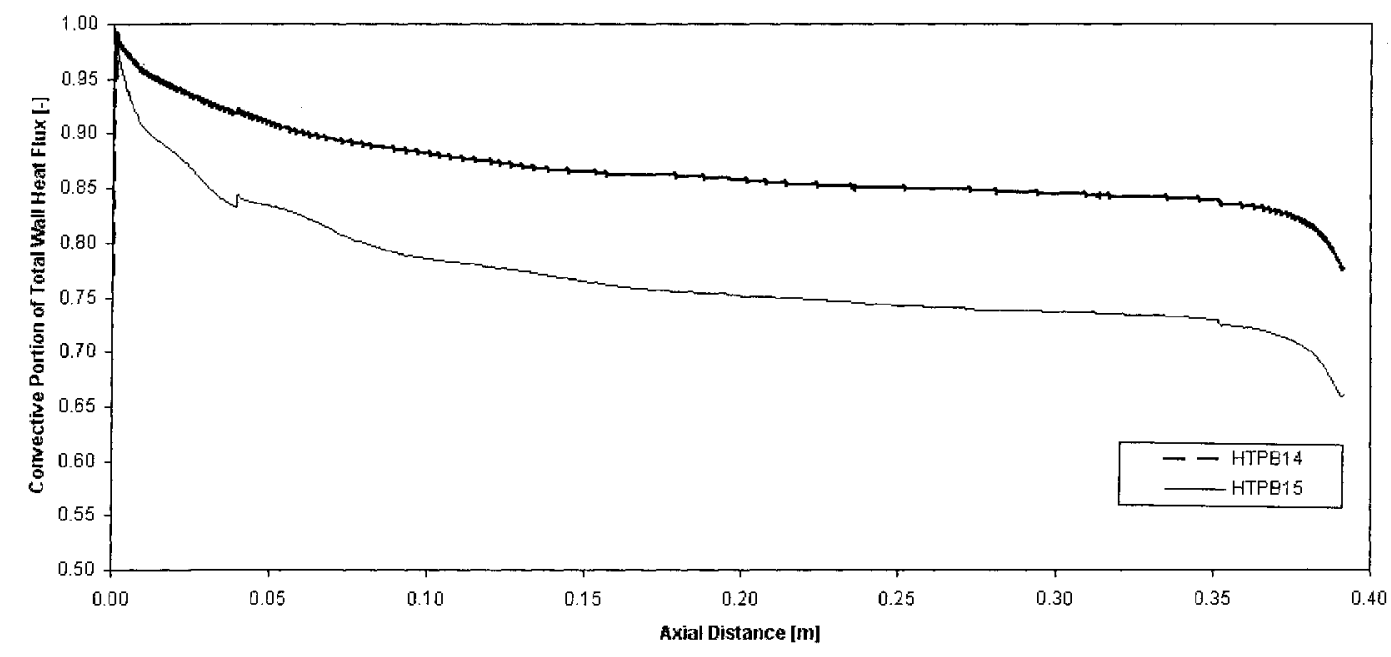

Figure 3.14: Contribution of convection to the total wall heat flux for HTPB14 and HTPB15 .

\subsubsection{Variable values at $0.5 \mathrm{~L}$}

The figures in this section were assembled at an axial length of 0.5L in the HTPB14 model.

Figure 3.15 shows the variation of temperature, axial velocity and gas mixture density as a function of distance from the wall. The approximate location of the flame is at the peak in the temperature profile. The velocity overshoot measured in combusting boundary layers (see Figure 3.1) is shown in this figure. This characteristic velocity overshoot is a result of the pressure gradient interaction with the lower density fluid in the flame region.

Figure 3.16 shows the mass fractions of each gas constituent as a function of distance from the wall. As expected, the oxygen mass fraction decreases completely 


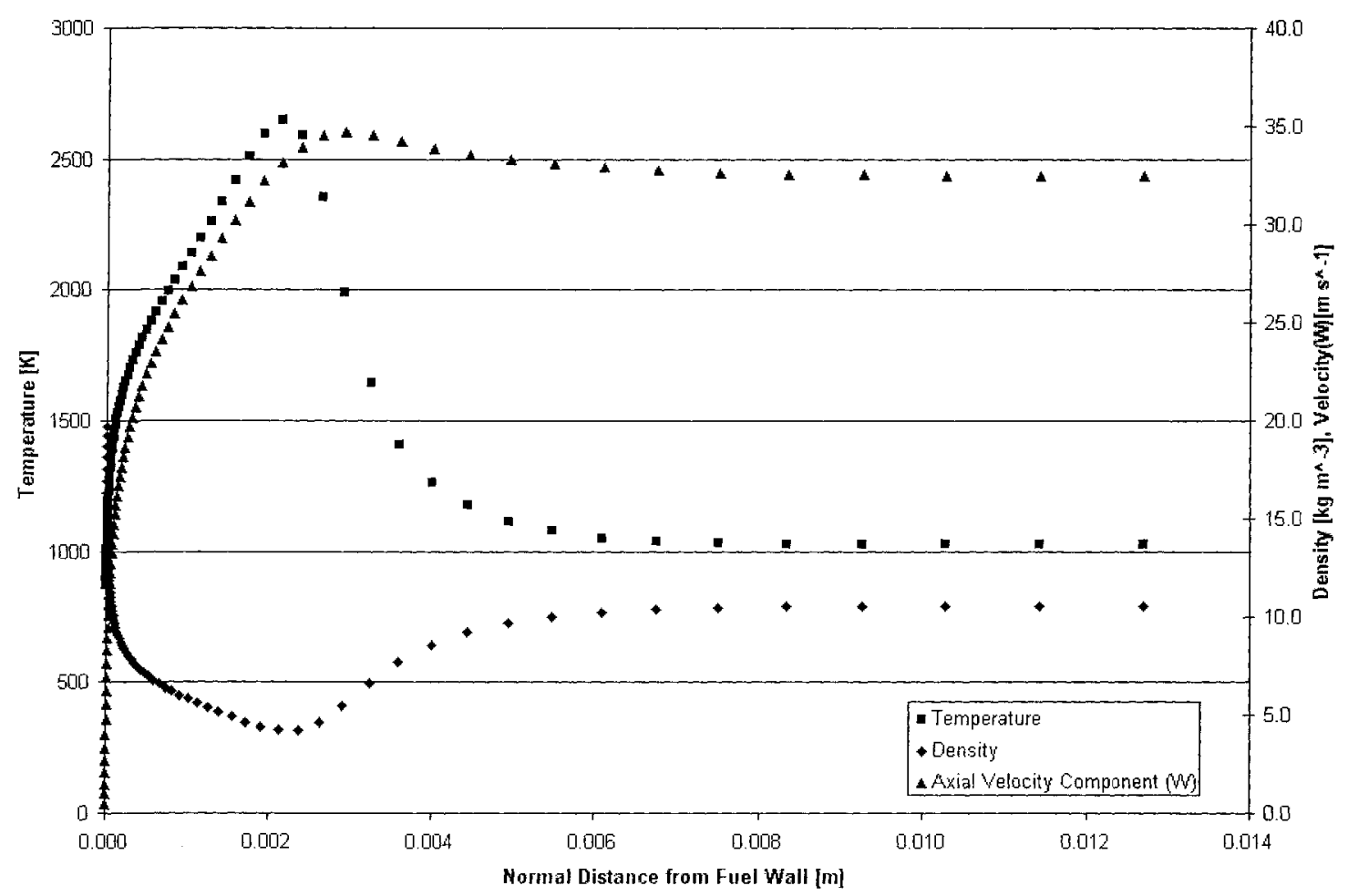

Figure 3.15: HTPB14 - Variation of density, temperature and axial velocity component normal to the fuel surface at $0.5 \mathrm{~L}$. 
to zero at the approximate flame location indicating complete oxidizer consumption below the flame. The oxygen mass fraction becomes constant at some distance from the wall, indicating unconsumed oxidizer in the free stream at $0.5 \mathrm{~L}$.

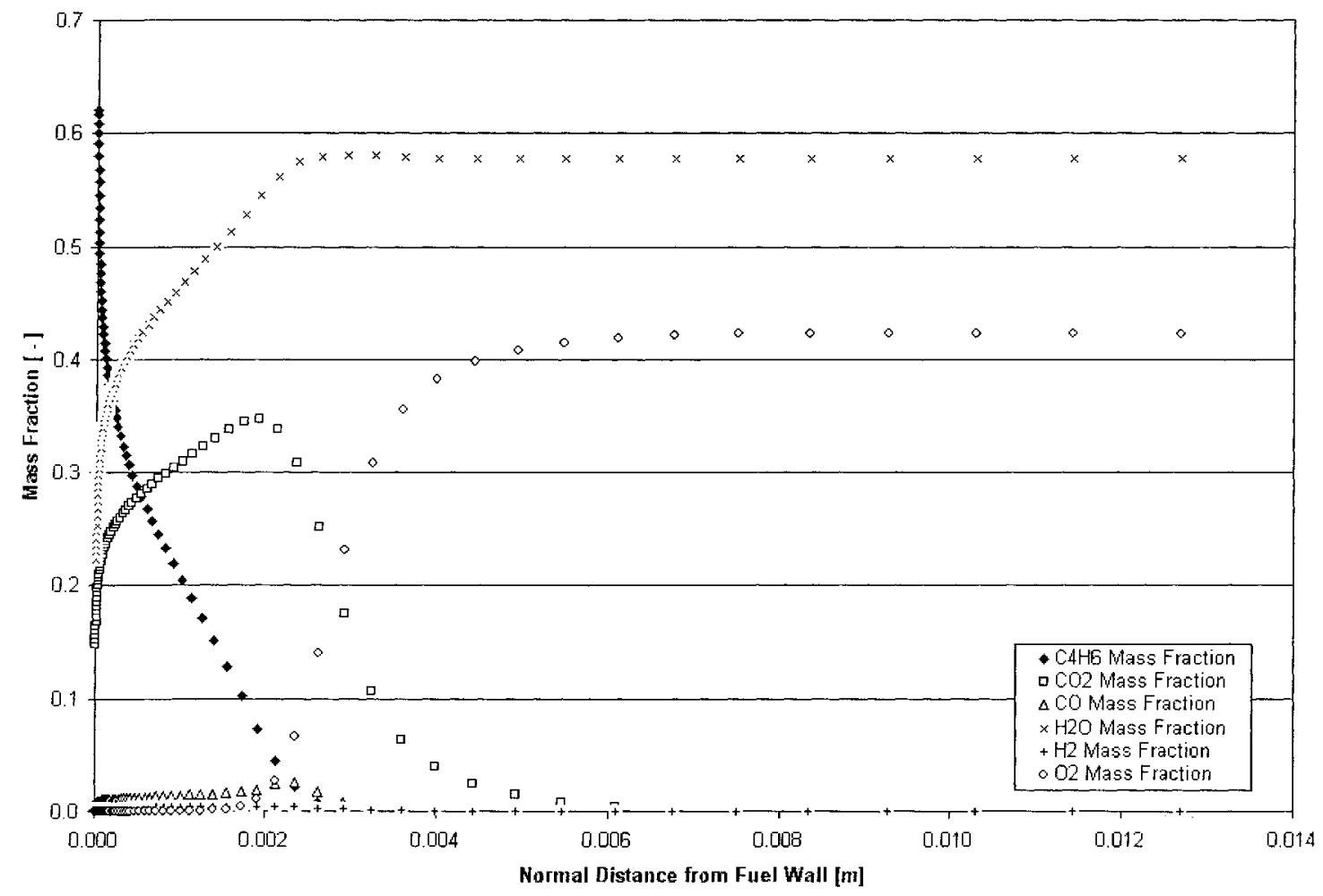

Figure 3.16: HTPB14 - Variation of component mass fractions normal to the fuel surface at $0.5 \mathrm{~L}$.

Figure 3.17 shows the variation of the fluid transport properties due to changes in temperature and fluid composition as a function of wall distance.

Figure 3.18 shows the variation in turbulence kinetic energy and turbulence energy dissipation, and the variation in the eddy viscosity calculated from those quantities. The eddy viscosity is seen to decrease in the vicinity of the flame and 


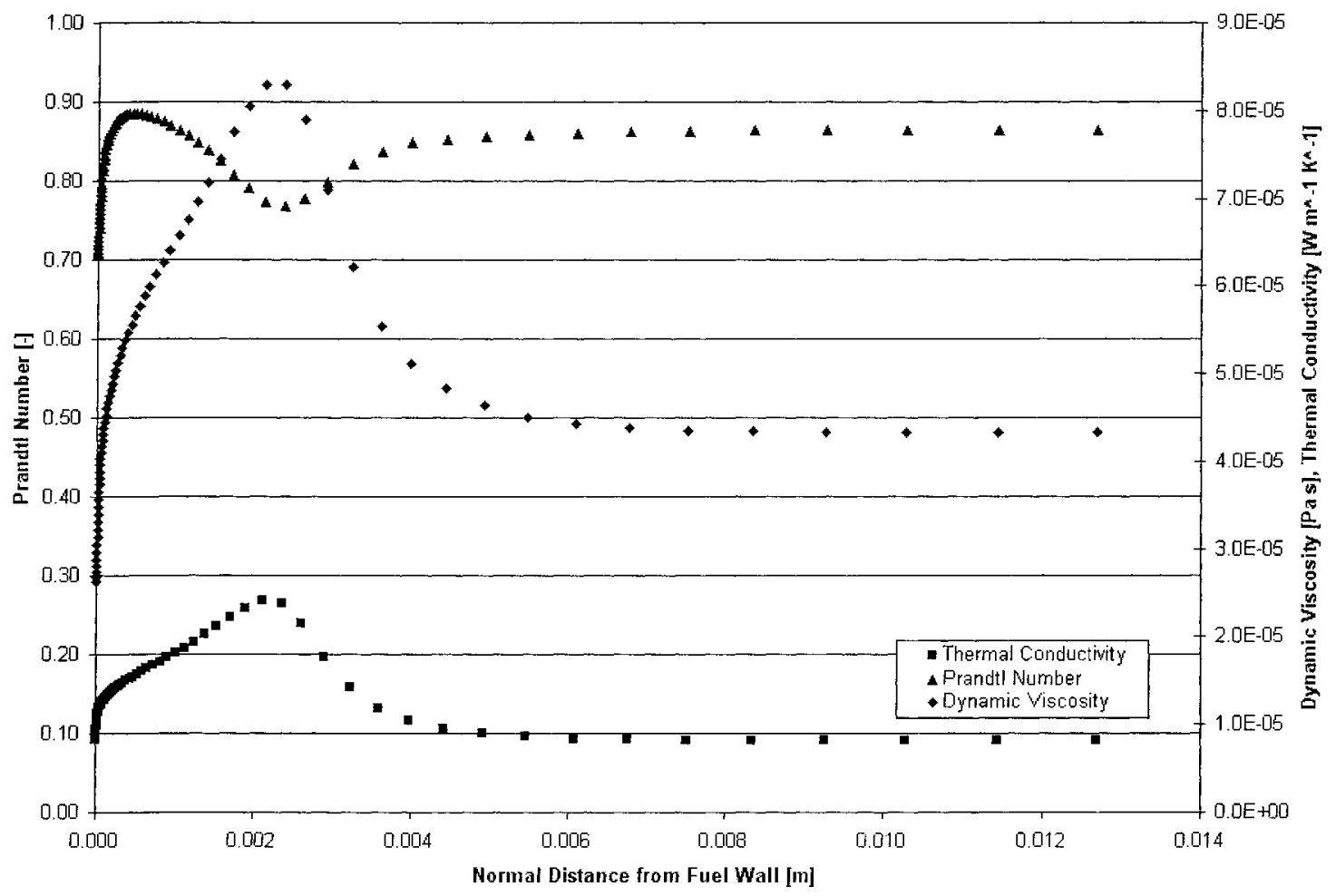

Figure 3.17: HTPB14 - Variation of dynamic viscosity, thermal conductivity and Prandtl number normal to the fuel surface at $0.5 \mathrm{~L}$. 
then come to a constant value away from the wall. This effect is mainly due to the decrease in density in the near flame region.

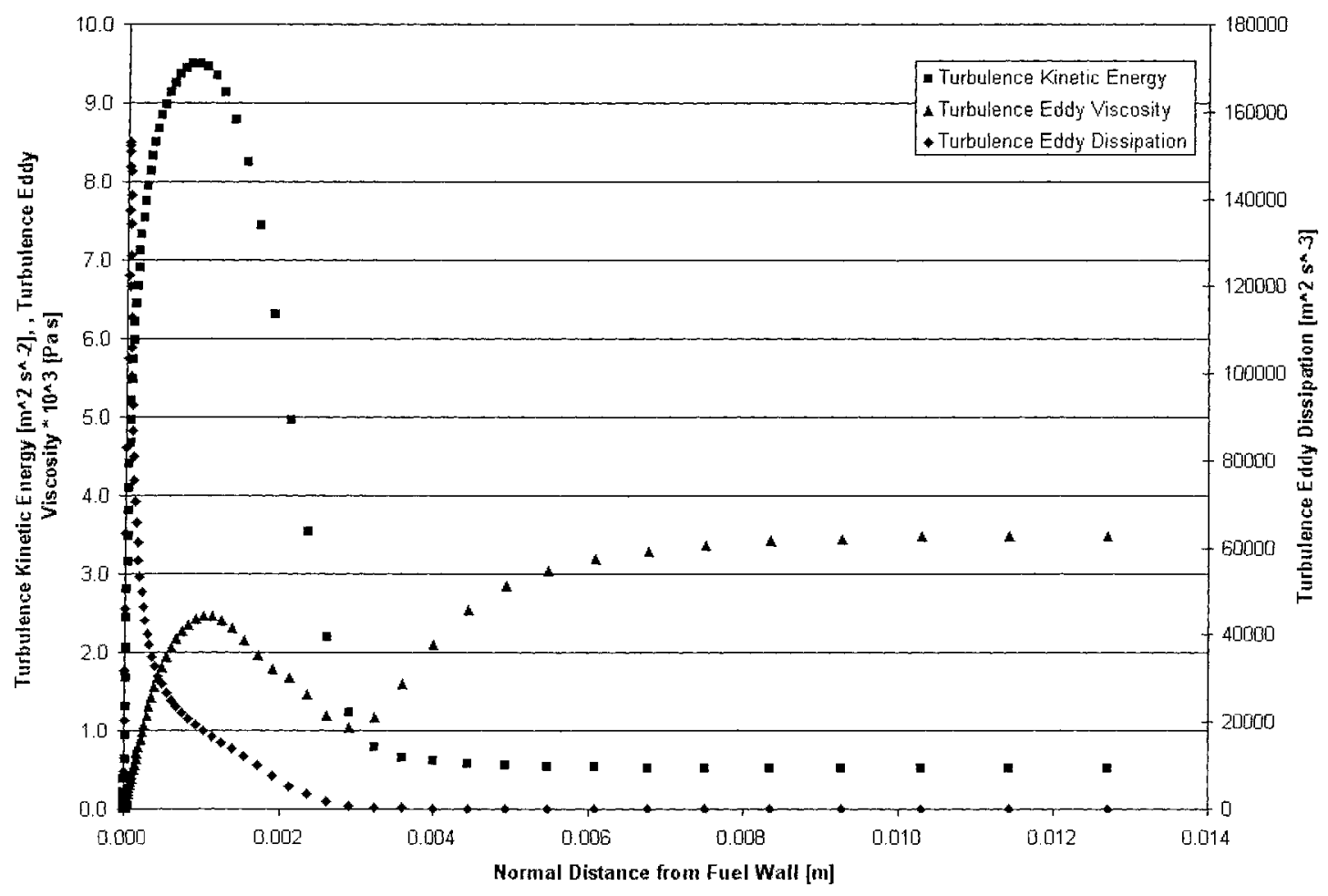

Figure 3.18: HTPB14 - Variation of turbulence kinetic energy, eddy dissipation and eddy viscosity normal to the fuel surface at $0.5 \mathrm{~L}$.

Figure 3.19 shows a comparison of the near wall temperature profiles for the both the HTPB14 and HTPB15 models. The flame in the HTPB15 model is further away from the wall in absolute terms than in the HTPB14 model because the port diameter of HTPB15 is larger than that of HTPB14. Because the temperature gradient is taken with respect to absolute distance, the steepness of the HTPB14 profile relative to that of the motor with the larger port, HTPB15, provides an 
explanation for the difference in the magnitude of the regression rate between the two models. The distance from the wall is made non-dimensional in Figure 3.20. In that figure the flame in the HTPB15 model is actually closer to the wall, due to the fact that both motors were the same length and therefore the boundary layer in HTPB15 has not developed to the same non-dimensional height as that in HTPB14.

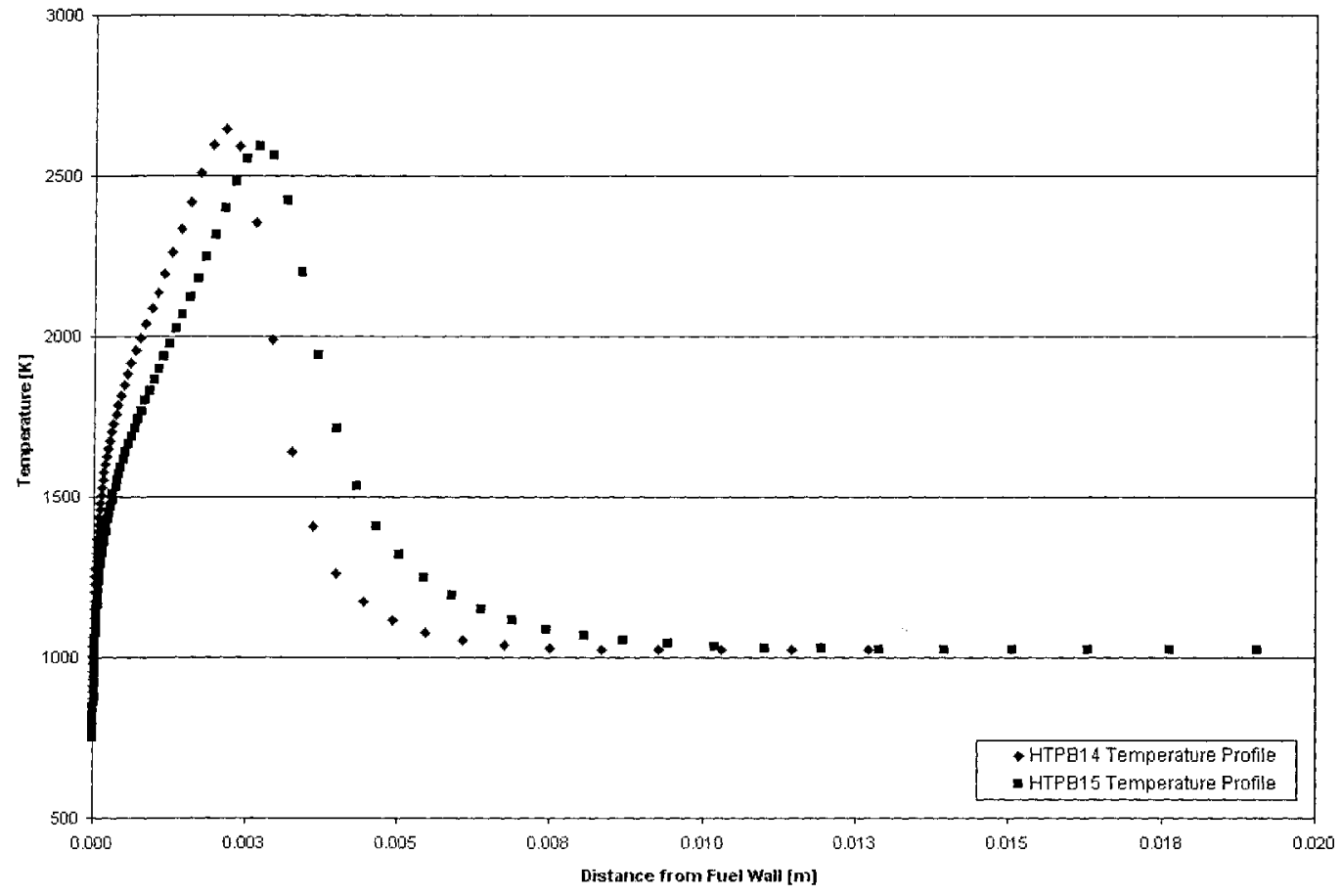

Figure 3.19: Comparison of temperature profiles as a function of absolute wall distance at $0.5 \mathrm{~L}$ for HTPB14 and HTPB15. 


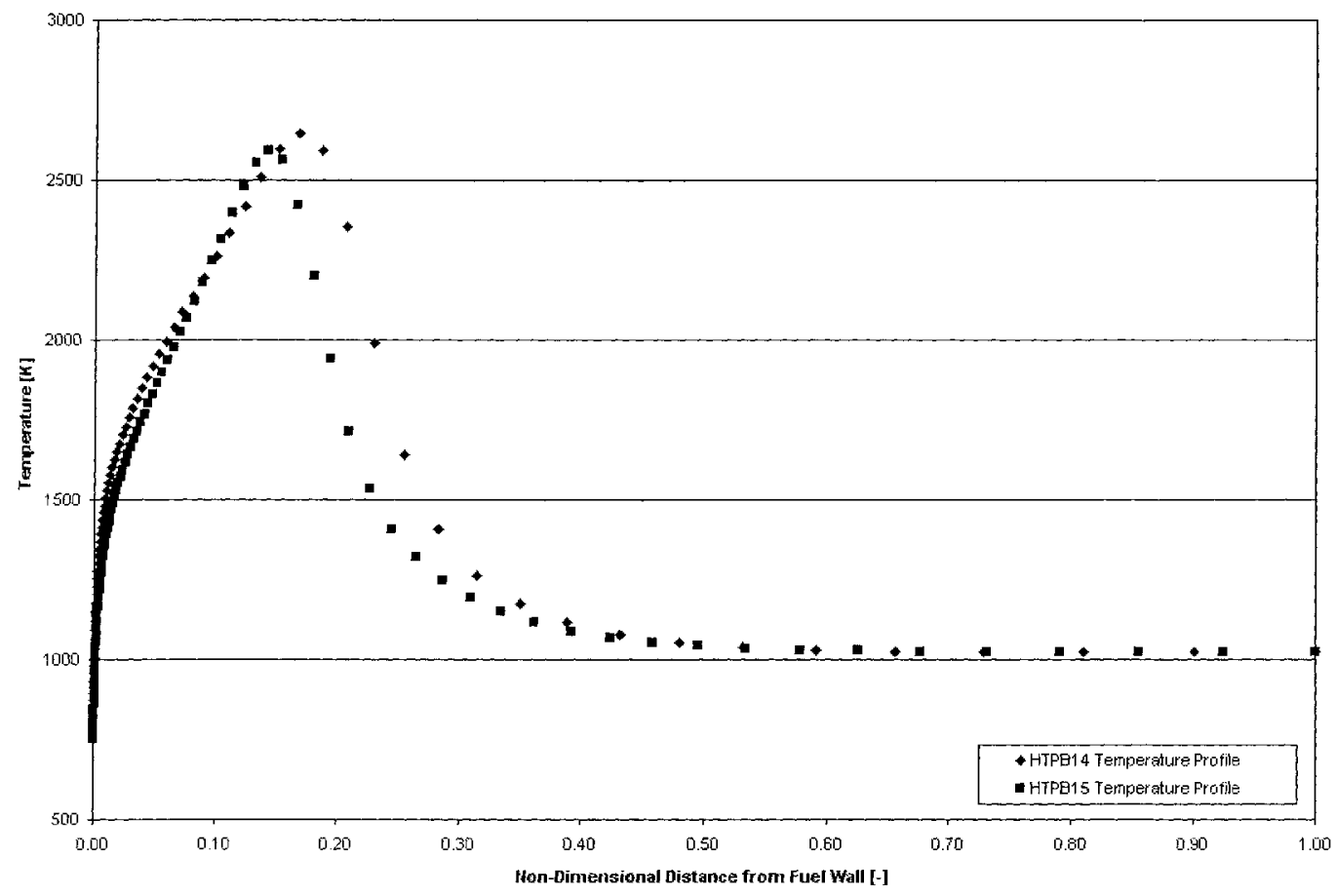

Figure 3.20: Comparison of temperature profiles as a function of non-dimensional wall distance at $0.5 \mathrm{~L}$ for HTPB14 and HTPB15. 


\subsubsection{Fuel regression rate, mass injection rate and surface temper- ature}

Due to the elimination of the precombustion chamber from the model geometry, the vortex on the fuel surface driven by the flow of oxidizer over the fuel step was not included in the flow field solution. The step was present because the precombustion chamber material was thinner than the web of the fuel grain. The oxidizer was expelled from the injector into the precombustion chamber and then flowed into the smaller diameter fuel grain. An image of this geometry is given in Figure 3.21, the vortex on the fuel grain is labeled vortex II. Relevant data for a comparison of the geometries of the HTPB14 and HTPB15 models to data taken from turbulent flows in pipe contractions from Reference [62] is given in Table 3.8. The effect of this vortex would be to increase the turbulent convective heat transfer to the fuel surface in the immediate vicinity of the reattachment zone. The attachment location of this recirculation zone was measured to be approximately $\mathrm{x} / D_{1}=0.5$ in the data cited. That distance corresponded to 0.6 source lengths along the fuel wall for the models, but is an approximation as the conditions in the models varied from those in the experiment presented in Ref. [62]. The regression rate increase due to this recirculation zone was not predicted in the current models.

Figures 3.22 and 3.23 show the regression rate, surface temperature and fuel mass injection profiles as a function of axial distance along the motor length. The 
Table 3.8: Data for step heights in HTPB14 and HTPB15 models.

\begin{tabular}{|l|l|l|}
\hline Source & $D_{2} / D_{1}$ & $\operatorname{Re}_{D_{1}}$ \\
\hline \hline HTPB14 & 0.5 & 436600 \\
HTPB15 & 0.8 & 217200 \\
Ref. [62] & 0.6 & 153800 \\
\hline
\end{tabular}

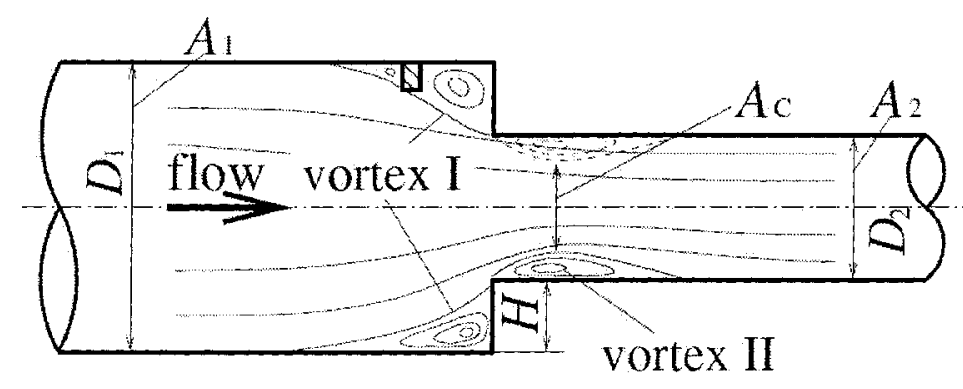

Figure 3.21: Vortex at fuel edge due to forward facing step geometry in motor.

experimentally measured temporally and spatially averaged regression rates and respective error bars are also shown on this figure as constant lines. Note that the experimental regression rate did vary in the axial direction, however this variation was not accurately quantified along the length of the grain and is not shown on the figure. The initial high regression rate predicted in both models was due to the high convective heat transfer to the fuel grain when the flame was very close to the fuel surface. The regression rate was seen to increase slightly with downstream distance, due to the increase in total wall heat flux with downstream distance shown previously. This effect has been measured experimentally and predicted numerically 
by researchers (see for example, Ref. [21], Ref. [63]).

The regression rate was over-predicted for Test 15 by HTPB15, and underpredicted for Test 14 by HTPB14. It is important to remember that these models were built using the motor operating conditions at the start of Tests 14 and 15, and the experimental data shown is the temporally and spatially averaged regression rate. As the burn progressed, the regression rate of the fuel would have decreased due to the decrease in total mass flux with the increasing port diameter. Therefore it is correct to say that the numerical results are an overestimation of the temporally averaged regression rate.

In an absolute sense, the regression rate predicted by the HTPB15 model compares more closely to the experimental data from Test 15 than does the HTPB14 model to the data from Test 14. One explanation is that the relative size of the source used for the mass injection in the HTPB15 model was smaller than that in the HTPB14 model due the larger diameter port in HTPB15. Since the flame was farther from the wall in the HTPB15 model, the physical error in the location of the fuel injection relative to the flame was lower, possibly resulting in higher accuracy of the calculated temperature gradient at the wall. Additionally, a larger portion of wall heat transfer in the larger diameter HTPB15 motor was due to radiation, and the radiative heat transfer to the wall is unlikely to be significantly affected by the use of subdomain sources to model the wall transpiration. Lastly, any increase 
in convective heat transfer due to the recirculation induced by the fuel step would have been much less in the HTPB15 model, due to a Reynolds number based on step height that was approximately half of that for the HTPB14 model. The neglect of the fuel step geometry had a lesser effect on the regression rate prediction obtained from HTPB15 than that for HTPB14.

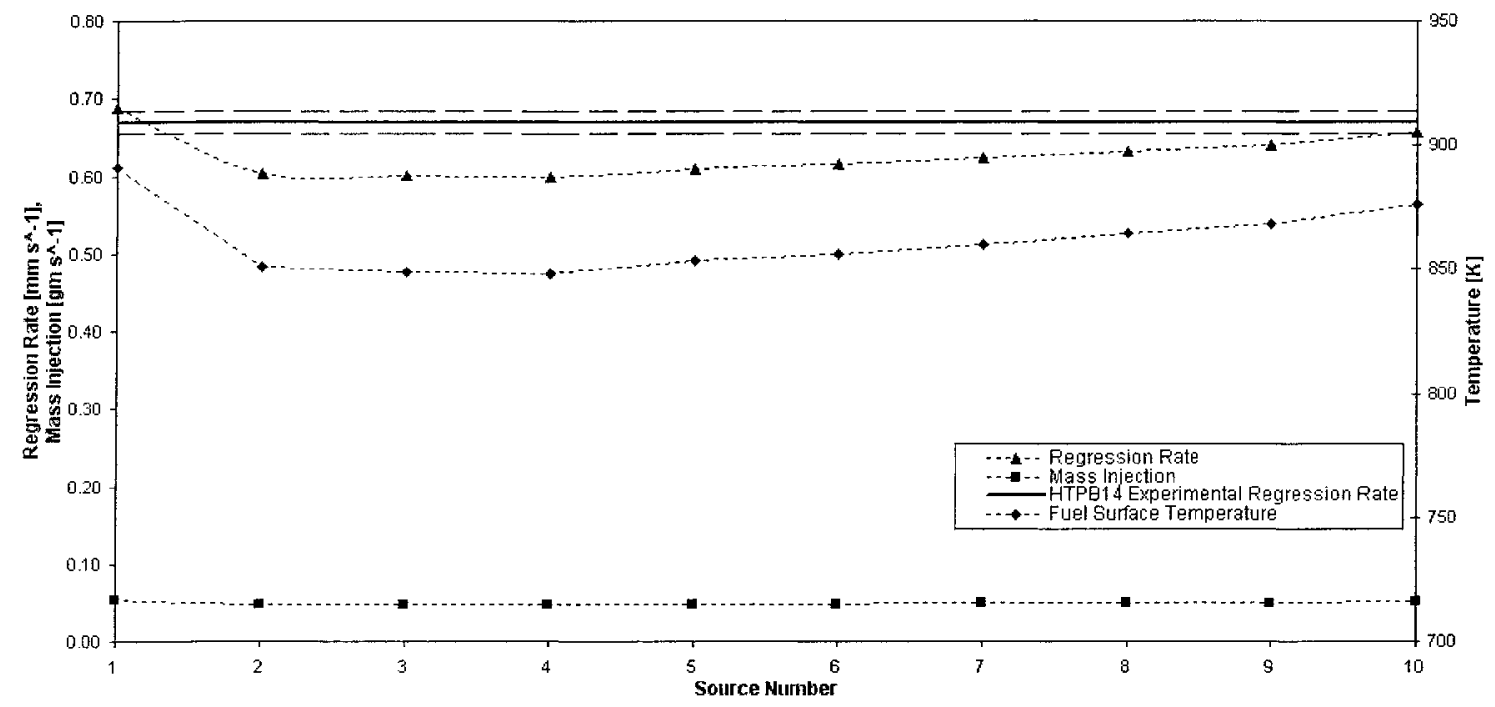

Figure 3.22: HTPB14 - Variation of regression rate, surface temperature and mass injection rate in the axial direction. Dashed lines indicate experimental error envelope.

\subsubsection{Comparison of numerical and experimental results}

Table 3.9 shows a summary of the regression rate calculated at each of the ten sources along the fuel wall, for both the HTPB14 and HTPB15 models, corresponding to the points in Figures 3.22 and 3.23. Also given are the spatially averaged regression rates for each model, the experimental regression rates for both tests, the experimental 


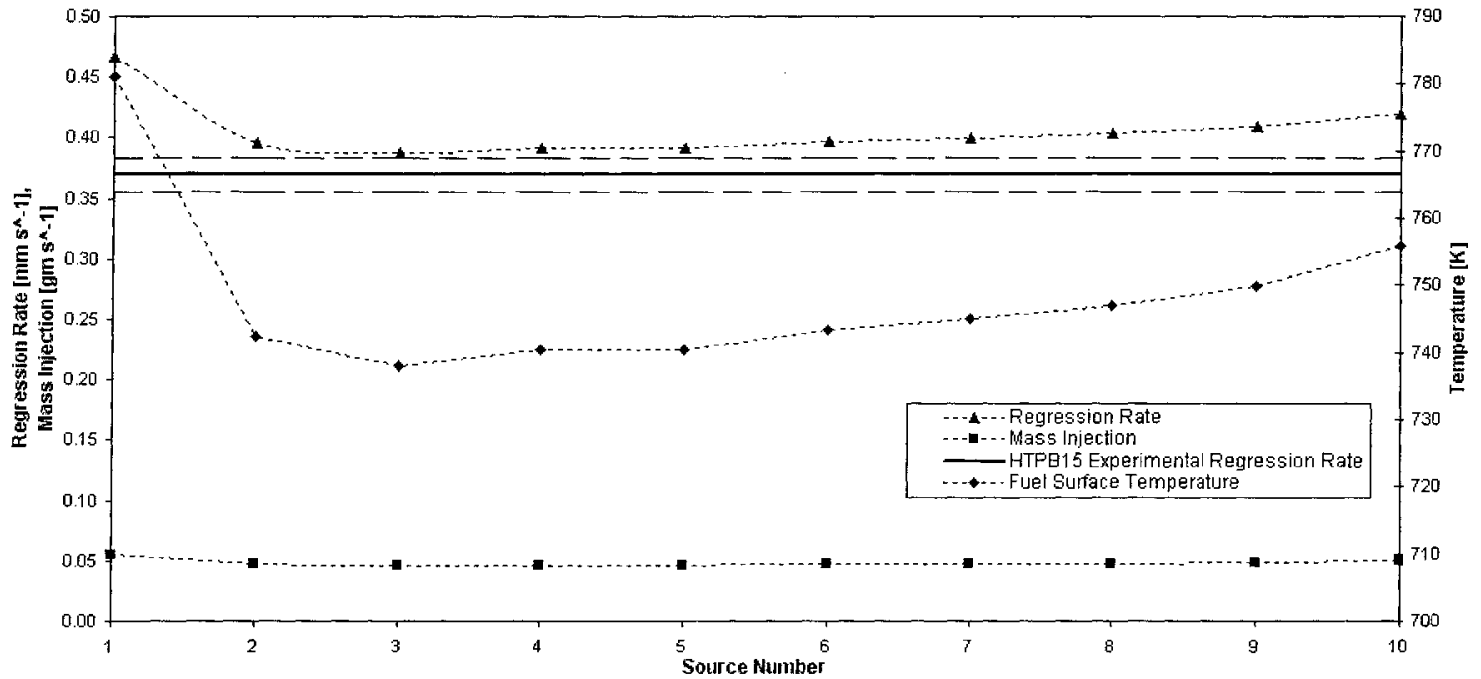

Figure 3.23: HTPB15 - Variation of regression rate, surface temperature and mass injection rate in the axial direction. Dashed lines indicate experimental error envelope.

errors for both tests, and the percent difference between the calculated spatially averaged regression rate and the experimental regression rate. It is important to remember that the regression rates were calculated for a motor operating at the initial test conditions after the start-up transient, and are an over prediction of the time average regression rate. Thus, the over prediction of the experimental regression rate by $\mathrm{HTPB} 15$ is an indication of a more accurate representation of the physics of the HRM than the that of the HTPB14 model.

A representative maximum flame temperature for the numerical model was taken as the maximum temperature in the entire domain. That allowed a loose comparison to the theoretical adiabatic flame temperature for the experimentally 
Table 3.9: Comparison of experimental and numerical results: regression rate.

\begin{tabular}{|l|l|l|}
\hline Model & HTPB14 & HTPB15 \\
\hline \hline Individual Sources & & \\
Source 1 & $0.689 \frac{\mathrm{mm}}{\mathrm{s}}$ & $0.466 \frac{\mathrm{mm}}{\mathrm{s}}$ \\
Source 2 & $0.604 \frac{\mathrm{mm}}{\mathrm{s}}$ & $0.395 \frac{\mathrm{mm}}{\mathrm{s}}$ \\
Source 3 & $0.601 \frac{\mathrm{mm}}{\mathrm{s}}$ & $0.387 \frac{\mathrm{mm}}{\mathrm{s}}$ \\
Source 4 & $0.599 \frac{\mathrm{mm}}{\mathrm{s}}$ & $0.392 \frac{\mathrm{mm}}{\mathrm{s}}$ \\
Source 5 & $0.609 \frac{\mathrm{mm}}{\mathrm{s}}$ & $0.392 \frac{\mathrm{mm}}{\mathrm{s}}$ \\
Source 6 & $0.615 \frac{\mathrm{mm}}{\mathrm{s}}$ & $0.397 \frac{\mathrm{mm}}{\mathrm{s}}$ \\
Source 7 & $0.624 \frac{\mathrm{mm}}{\mathrm{s}}$ & $0.400 \frac{\mathrm{mm}}{\mathrm{s}}$ \\
Source 8 & $0.637 \frac{\mathrm{mm}}{\mathrm{s}}$ & $0.403 \frac{\mathrm{mm}}{\mathrm{s}}$ \\
Source 9 & $0.641 \frac{\mathrm{mm}}{\mathrm{s}}$ & $0.408 \frac{\mathrm{mm}}{\mathrm{s}}$ \\
Source 10 & $0.657 \frac{\mathrm{mm}}{\mathrm{s}}$ & $0.419 \frac{\mathrm{mm}}{\mathrm{s}}$ \\
\hline Numerical (Avg.) & $0.627 \frac{\mathrm{mm}}{\mathrm{s}}$ & $0.406 \frac{\mathrm{mm}}{\mathrm{s}}$ \\
Experimental & $0.669 \frac{\mathrm{mm}}{\mathrm{s}}$ & $0.369 \frac{\mathrm{mm}}{\mathrm{s}}$ \\
\hline Difference & $-0.042 \frac{\mathrm{mm}}{\mathrm{s}}$ & $0.037 \frac{\mathrm{mm}}{\mathrm{s}}$ \\
Percent Difference & $-6.3 \%$ & $10.0 \%$ \\
\hline Experimental Error & $0.015 \frac{\mathrm{mm}}{\mathrm{s}}$ & $0.014 \frac{\mathrm{mm}}{\mathrm{s}}$ \\
\hline
\end{tabular}

measured OF ratio, as presented in Table 3.10. The flame temperature varied along the length of the domain due to changing OF ratios along the length of the combustion chamber. The variation of OF ratio in a hybrid motor is due to the variation of regression rate along the length of the motor. Many assumptions were made in the combustion model: turbulent mixing rate and Arrhenius rate limited chemistry, simplification of the products of HTPB pyrolysis, and a simplified reaction model. Additionally, the theoretical temperatures in Table 3.10 were calculated using PROPEP for the temporally and spatially averaged OF ratio during the static tests, not the instantaneous values at the flame. 
Table 3.10: Comparison of experimental and numerical results: maximum flame temperature.

\begin{tabular}{|l|l|l|}
\hline Model & HTPB14 & HTPB15 \\
\hline \hline Numerical & $2664 \mathrm{~K}$ & $2602 \mathrm{~K}$ \\
Theoretical, adiabatic & $2645 \mathrm{~K}$ & $2793 \mathrm{~K}$ \\
\hline
\end{tabular}

Table 3.11 presents the approximate flame location for both models, as determined by the peak temperatures of the profiles in Figure 3.19. CFD results for a axisymmetric hybrid motor with a $0.0168 \mathrm{~m}$ port diameter operating with $\mathrm{HTPB} / \mathrm{GOX}$ at $565 \mathrm{~kg} / \mathrm{m}^{2} \mathrm{~s}, \mathrm{P}_{c}=60 \mathrm{~atm}, \mathrm{~L} / \mathrm{D}=35$ are also presented in this table. The results were approximated from a published graph of temperature profile as a function of wall distance at $0.5 \mathrm{~L}$ and are therefore somewhat subjective; they are included for general comparison purposes here.

Table 3.11: Comparison of experimental and numerical results: flame location at $0.5 \mathrm{~L}$.

\begin{tabular}{|l|l|l|}
\hline Model & HTPB14 & HTPB15 \\
\hline \hline Numerical & $0.0021 \mathrm{~m}$ & $0.0027 \mathrm{~m}$ \\
Results from Ref.[21] & $0.0018 \mathrm{~m}$ & \\
\hline
\end{tabular}




\section{Chapter 4}

\section{Summary and Conclusions}

\subsection{Experimental Results}

The $60 \mathrm{Al} \% / 40 \% \mathrm{HTPB} / 90 \%$ HP propellant combination exhibited combustion instability during Tests 1-4, all of which had average chamber pressures $<400$ psia. This instability was eliminated both by use of a turbulator or mixing device just upstream of the fuel grain to induce recirculation during Test 6 , and also by increasing the average chamber pressure to $>570$ psia during Tests 7 and 8 . No combustion instabilities were observed with either the $60 \% \mathrm{AlMg} / 40 \% \mathrm{HTPB} / 90 \% \mathrm{HP}$ or the HTPB $/ 90 \%$ HP propellant combinations. The instabilities were attributed to a phenomenon called "chuffing", or periodic shedding of a molten aluminum oxide $\left(\mathrm{Al}_{2} \mathrm{O}_{3}\right)$ layer that had accumulated on the grain surface.

The average combustion efficiency measured for the $60 \% \mathrm{Al} / 40 \% \mathrm{HTPB} / 90 \% \mathrm{HP}$ propellant during Tests 1 and 2, without a reaction chamber in the motor, was 
73.0\%. A reaction chamber was added subsequent to these tests to increase the propellant residence time, promoting more complete combustion. The measured combustion efficiency for Tests 3-8 ranged from $72.8 \%-89.5 \%$, with the average being $82.8 \%$. The combustion efficiency of Test 6 with the turbulator insert was $88.8 \%$, $6.0 \%$ higher than the average. The range of combustion efficiencies measured for the $60 \% \mathrm{AlMg} / 40 \% \mathrm{HTPB} / 90 \% \mathrm{HP}$ propellant combination were $74.4 \%-86.7 \%$ with the average being $81.0 \%$. The combustion efficiencies measured for the HTPB $/ 90 \%$ propellant combination were $78.8 \%$ and $90.1 \%$ with the average being $84.5 \%$.

The $60 \% \mathrm{Al} / 40 \% \mathrm{HTPB} / 90 \% \mathrm{HP}$ combination exhibited pressure dependent combustion efficiency; the average efficiency measured for Tests 7,8 was $88.6 \%$, $11.5 \%$ higher than the average efficiency measured for Tests $3,4,5$ of $77.0 \%$. This effect could be attributed to the pressure dependence of aluminum particle burn time of $t_{b} \sim P^{-0.2}$. The combustion efficiencies for the high pressure tests with $60 \% \mathrm{AlMg} / 40 \% \mathrm{HTPB} / 90 \% \mathrm{HP}$ (Tests 11,12 ) had an average of $82.9 \%, 3.1 \%$ higher than that of the lower pressure tests (Tests $9,10,13$ ). However, this value was very close to the experimental error on the combustion efficiency parameter so this result was inconclusive. The chamber pressure of the two HTPB $/ 90 \% \mathrm{HP}$ tests was not varied.

The effect of metal type on combustion efficiency was difficult to delineate as the particle size of the AlMg alloy was substantially larger than that of 
the $\mathrm{Al}$ alloy used in the HyperG tests. The average combustion efficiency of the high pressure $60 \% \mathrm{Al} / 40 \% \mathrm{HTPB} / 90 \% \mathrm{HP}$ tests was $5.7 \%$ greater than that of the $60 \% \mathrm{AlMg} / 40 \% \mathrm{HTPB} / 90 \% \mathrm{HP}$ tests possibly due to the larger particle size of the AlMg alloy, which would theoretically have had a longer burn time than that of the much smaller $\mathrm{Al}$ particles.

Graphs of the combustion efficiency for each propellant combination and the theoretical characteristic velocity as a function of the experimental OF ratio showed a trend of increasing combustion efficiency towards to the oxidizer rich portion of the figure. However, a larger number of tests are required to draw quantitative conclusions regarding the effect of OF ratio on combustion efficiency for the propellant combinations tested.

The average ISP measured for Tests $3-8$ with the $60 \% \mathrm{Al} / 40 \% \mathrm{HTPB} / 90 \% \mathrm{HP}$ combination was $196.4 \mathrm{~s}$. That for $60 \% \mathrm{AlMg} / 40 \% \mathrm{HTPB} / 90 \% \mathrm{HP}$ was $193.6 \mathrm{~s}$ and finally for HTPB $/ 90 \% \mathrm{HP}$ it was $199.8 \mathrm{~s}$. Note that the ISP of the propellant is affected by combustion performance $\left(c^{*}\right)$ as well as nozzle losses; the two performance parameters are not mutually exclusive. Significant two phase flow through the nozzle during the tests with metallized propellants contributed heavily to the ISP losses.

The average regression rate measured for the $60 \% \mathrm{Al} / 40 \% \mathrm{HTPB} / 90 \% \mathrm{HP}$ combination was $0.77 \mathrm{~mm} / \mathrm{s}$, with a range of $0.53 \mathrm{~mm} / \mathrm{s}-1.10 \mathrm{~mm} / \mathrm{s}$. The av- 
erage regression rate for the $60 \% \mathrm{AlMg} / 40 \% \mathrm{HTPB} / 90 \% \mathrm{HP}$ combination was 0.98 $\mathrm{mm} / \mathrm{s}$, with a range of $0.70 \mathrm{~mm} / \mathrm{s}-1.27 \mathrm{~mm} / \mathrm{s}$. The average regression rate measured for the HTPB $/ 90 \% \mathrm{HP}$ combination was $0.52 \mathrm{~mm} / \mathrm{s}$, with values of $0.37 \mathrm{~mm} / \mathrm{s}$, and $0.67 \mathrm{~mm} / \mathrm{s}$.

The regression rate of the $60 \% \mathrm{AlMg} / 40 \% \mathrm{HTPB} / 90 \% \mathrm{HP}$ combination was consistently approximately $0.2 \mathrm{~mm} / \mathrm{s}$ higher than that of the $60 \% \mathrm{Al} / 40 \% \mathrm{HTPB} / 90 \% \mathrm{HP}$ combination. The correlation of regression rate with total mass flux for the two metallized propellant combinations yielded mass flux exponents of 0.64 for $60 \% \mathrm{AlMg} / 40 \% \mathrm{HTPB} / 90 \% \mathrm{HP}$ and 0.74 for $60 \% \mathrm{Al} / 40 \% \mathrm{HTPB} / 90 \% \mathrm{HP}$. These exponents indicated a deviation from traditional turbulent convective heat transfer dominated regression rate behaviour, likely due to the pressure dependence of the radiative portion of the wall heat transfer. Additional experimental data for motors operating at various chamber pressures and total mass flux levels are required to draw conclusions about the influence of chamber pressure on regression rate for the propellant combinations tested in the HyperG study.

The regression rate, and thus the ISP, would be affected by a scale up of these laboratory motors to commercial sizes. While the laboratory scale tests yield information about the performance of the metallized HTPB/HP propellant combinations relative to others, performance data for the large scale motor would have to 
be obtained using experiments, numerical models, or both.

\subsection{Numerical Results}

The assumptions that were made during the construction of the model and its limitations are summarized below so that the conclusions reached in this section can be taken in context.

1. The injection of fuel in a subdomain region of one element $(0.001 \mathrm{~mm})$ thickness along the wall likely resulted in an under prediction of $\left.\frac{\partial T}{\partial x_{i}}\right|_{\text {wall }}$.

2. The geometry of the precombustion chamber upstream of the fuel grain was not included in the model, therefore the effects of the recirculation zone formed at the start of the fuel grain on the wall heat transfer and flow field were not resolved.

3. A steady-state solution was computed using the chamber pressure and port diameter measured at the start of the burn, therefore the results are valid only for the initial operating conditions of motor and have assumed that the inherently transient physical process can be analyzed at one "snap shot" in time.

4. Axisymmetric boundary conditions were used so any three dimensional characteristics of the flow field were neglected. 
5. The conductive heat loss through the fuel was accounted for in the fuel surface energy balance in a one-dimensional manner, under the assumption that the outer diameter of the fuel was at ambient temperature, which is strictly valid only during the initial portion of the motor operation.

6. The products of HTPB pyrolysis were simplified to include only 1,3-butadiene.

7. It was assumed that the hydrogen peroxide was fully decomposed upon exit from the catalyst pack, and that the decomposition process was adiabatic.

8. The contribution of soot to the radiative heat transfer was neglected resulting in an under prediction of the total heat transfer to the fuel grain.

9. The molecular diffusion coefficient was approximated using the molecular viscosity for each fluid component.

10. The chamber pressure measured during the test was used as the reference pressure in the model resulting in a slight over prediction of the pressure distribution in the motor.

11. The combustion process was approximated by a simplified two step reaction and the reaction rates were modeled using the Eddy Dissipation / Finite Rate Model, which affected the prediction of flame thickness, location, and maximum temperature. 
12. A finite number of subdomain sources were used to resolve the variation of temperature and regression rate along the fuel surface.

The main goals of the numerical model were to predict the regression rate of the non-metallized HTPB fuel at the start of Tests 14 and 15, and to predict the spatial variation in the regression rate.

The spatially averaged numerical values differed from the temporally and spatially averaged experimental values by $-6.3 \%$ and $10.0 \%$ for the HTPB14 and HTPB15 models respectively. The regression rate predicted for HTPB15 was higher than that obtained during Test 15 which follows from the fact that the numerical model was solved for the initial operating conditions of the respective tests. However, the regression rate predicted for the HTPB14 model was lower than that measured during Test 14, even though it too was solved for the initial operating conditions of that test. The reasons for this are two fold. First, because the precombustion chamber was not included in the geometry of the model, the recirculation zone on the fuel surface at the start of the grain was not modeled, resulting in an under estimation of the heat transfer to the fuel surface in this region. The geometry of the HTPB14 model was such that this effect was much more prominent in that model than in HTPB15. Secondly, convective heat transfer was a larger portion of the total wall heat transfer for the HTPB14 model as it had a larger mass flux. Thus, the injection of the fuel in a finite volume along the wall, rather than at the 
nodes along the wall, had a larger affect on the regression rate predicted by the HTPB14 model.

Both models predicted an increase in regression rate with increasing downstream distance after an initial high regression rate region immediately downstream of the port inlet. The interesting characteristics of a turbulent boundary layer with blowing and combustion were observed: velocity overshoot, kinetic energy overshoot and the variation of the eddy viscosity parameter as a result. Finally, the results of the model shed insight into the relative importance of radiative heat transfer at different port diameters in an HRM using HP oxidizer, and demonstrated the scaling of the regression rate with total mass flux.

\subsection{Recommendations}

\subsubsection{Experimental program}

The following recommendations apply to the experimental HyperG program funded by DND Canada.

1. Move to hydride (possibly $\mathrm{LiAlH}_{4}$ or even $\mathrm{Li}_{3} \mathrm{AlH}_{6}$, see Ref. [64]) type fuels to increase the attainable fuel regression rate and therefore develop a fuel that is feasible for high thrust applications. Substantial research and development will likely be needed to develop manufacturing methods for this fuel grain. 
2. Add a turbulator or mixing diaphragm (as in the experiments reported by Osmon [7] and Lips [11]) at the downstream end of the fuel grain to promote turbulent mixing in the reaction chamber, with the intent of increasing the combustion efficiency of the motor.

3. Test hydrides with magnesium and aluminum/magnesium alloy metal additives with similar and small particle sizes $(<10 \mu \mathrm{m})$.

4. Perform static tests at set oxidizer mass flux levels spanning a range of values, with multiple chamber pressures tested at each value, to identify pressure dependency of the regression rate in different mass flux regimes. This will provide a complete map of the regression rate characteristics of the propellant combination in operating ranges useful for launch applications.

The results of these tests will give a good indication of the performance capabilities of hydride type fuels with hydrogen peroxide, and the suitability of this propellant combination for launch applications.

\subsubsection{Numerical model}

The numerical model presented in this work represents a foundation for the construction of a more robust model, that will provide a reliable predictive capability for the design of hybrid rocket motors operating with polymer fuels and gaseous ox- 
idizers. The following recommendations apply to the continuation of the numerical modeling program.

1. Replace the subdomain continuity sources along the wall with continuity sources applied directly at the fuel wall using the new boundary source capability in ANSYS CFX 10.0.

2. Model the entire motor including the precombustion and reaction chambers, and nozzle.

3. Implement the fluid-structure interaction capability in ANSYS CFX 10.0 to physically move the mesh at the fuel wall during a transient simulation of the problem, solving the time dependent flow field and determining the variation of the regression rate temporally in addition to spatially.

4. Mesh the solid fuel and solve the energy equation in the fuel using CFX conjugate heat transfer capability.

5. Solve the three dimensional flow field.

These additions will bring the numerical model much closer to being a useful design tool for a polymer/hydrogen peroxide hybrid rocket motor with predictive performance capability. 


\section{Bibliography}

[1] G. Sutton, O. Biblarz. "Rocket Propulsion Elements," 7th Ed., John Wiley and Sons Inc., 2001, Chapter 2, 3, 15.

[2] A. J. Cesaroni, Hydro Reactive Gas Generator Hybrids: HyperG, Internal Report No. 2, Cesaroni Technology Inc., Gormley, Ontario, Sept. 14, 2002.

[3] E. J. Wernimont and S. D. Heister, Combustion experiments in hydrogen peroxide/polyethylene hybrid rocket with catalytic ignition, Journal of Propulsion and Power, Vol. 16, No. 2, 2000, pp. 318-326.

[4] E. J. Wernimont and S. E. Meyer, Hydrogen peroxide hybrid rocket engine performance investigation, AIAA Paper 94-3147, June 1994.

[5] R. W. Humble, M. P. Bettner, R. A. Sandfry, Hystar hybrid rocket program at the United States Air Force Academy, AIAA Paper 1997-2797, July 1997. 
[6] G. K. Lund, D. W. Starrett, and K. C. Jensen, Development and lab scale testing of a gas generator hybrid fuel in support of the hydrogen peroxide hybrid upper stage program, AIAA Paper 2001-3244, July 2001.

[7] R. V. Osmon, An experimental investigation of a lithium aluminum hydride - hydrogen peroxide hybrid rocket Aerospace Chemical Engineering, Chemical Engineering Progress Symposium Series, Vol. 62, No. 61, 1966, pp. 92-102.

[8] G. A. Marxman, C. E. Wooldridge, R. J. Muzzy, Fundamentals of hybrid boundary layer combustion, Heterogenous Combustion, Progress in Astronautics and Aeronautics, Vol. 15, Academic Press Inc, 1964, pp. 485-521.

[9] G. A. Marxman, M. Gilbert, Turbulent boundary layer combustion in the hybrid rocket, Ninth International Symposium on Combustion, The Combustion Institute, Academic Press Inc., 1964, pp. 371-383.

[10] L. Lees, Convective heat transfer with mass addition and chemical reactions, Combustion and Propulsion, Third AGARD Colloquium, Pergamon Press Inc., 1958 , p. 451 (in [1], pp. 736).

[11] H. R. Lips, Experimental investigation on hybrid rocket engines using highly aluminized fuels, Journal of Spacecraft and Rockets, Vol. 12, No. 9, September 1997, pp.539-545. 
[12] H. R. Lips, Metal combustion in high performance hybrid rocket propulsion systems, AIAA Paper 76-640, July 1976.

[13] L. D. Strand, R. L. Ray, Hybrid rocket combustion study, AIAA Paper 93-2412, 1993.

[14] L.D. Strand, M. D. Jones, R. L. Ray, N. S, Cohen, Characterization of hybrid rocket internal heat flux and HTPB fuel pyrolysis. AIAA Paper 94-2876, 1994.

[15] L. D. Smoot, C. F. Price, Regression rates of nonmetalized hybrid fuel systems. AIAA Journal, Vol. 3, No. 8, 1965, pp. 1406-1413.

[16] L. D. Smoot, C. F. Price, Regression rates of metalized hybrid fuel systems, AIAA Journal, Vol. 4, No. 5, 1966, pp. 910-956.

[17] M. J. Chiaverini, N. Serin, D. K. Johnson, Yeu-Cheng Lu, K. K. Kuo, G. A. Risha, Regression rate behaviour of hybrid rocket solid fuels, Journal of Propulsion and Power, Vol. 16, No. 1, 2000, pp.125-132,

[18] N. Serin, Y. Gogus, Navier-Stokes investigation on reacting flow field of HTPB/O2 hybrid motor and regression rate evaluation, AIAA Paper 2003-4462, 2003.

[19] B. Leckner, Spectral and total emissivity of water vapour and carbon dioxide, Combustion and Flame, Vol. 19, 1972, pp. 33-48. 
[20] G. C. Cheng, R. C. Farmer, H. S. Hones, J. S. McFarlane, Numerical simulation of the internal ballistics of a hybrid rocket motor, AIAA Paper 94-0554, 1994.

[21] S. Venkateswaran and C. L. Merkle, Size scale-up in hybrid rocket motors, AIAA Paper 96-0647, 1996.

[22] M. J. Chiaverini, G. C. Harting, Yeu-Cheng Lu, K. K. Kuo, A. Peretz, S. H. Jones, B. S. Wygle, J. P. Arves, Pyrolysis behaviour of hybrid-rocket solid fuels under rapid heating conditions, Journal of Propulsion and Power, Vol. 15, No. 7, November-December 1999, pp.888-895.

[23] M. J. Chiaverini, K. K. Kuo, A. Peretz, G. C. Harting, Regression-rate and heat-transfer correlations for hybrid rocket propulsion, Journal of Propulsion and Power, Vol. 17, No. 1, 2001, pp. 99-110.

[24] H. Loh, M. Golafshani, Computation of viscous chemically reacting flows in hybrid rocket motors using an upwind LU-SSOR scheme. AIAA Paper 90-1570, June, 1990 .

[25] D. R. Cruise, Theoretical computations of equilibrium compositions, thermodynamic properties, and performance characteristics of propellant systems, NWC TP 6037. 
[26] FMC, Chemical Products Group, Technical Bulletin Number 46, Bayport, Texas, USA, 2001.

[27] FMC Chemical Products Group, Safety and Handling of 90\% Hydrogen Peroxide, Presentation at CTI, 2004.

[28] J. Louwers, A. J. Cesaroni, Hydro Reactive Gas Generator Hybrids: HyperG, Internal Report No. 3, Cesaroni Technology Inc., Gormley, Ontario, Feb. 5, 2003.

[29] E. J. Wernimont, S. D. Heister, Reconstruction technique for reducing hybridrocket combustion test data, Journal of Propulsion and Power, Vol. 15, No. 1, 1999, pp. 128-136.

[30] B, Greiner, R. A. Frederick Jr., Hybrid rocket instability, AIAA Paper 93-2553, 1993.

[31] D. Hanson-Parr, T. Parr, Thermal properties measurements of solid rocket propellant oxidizers and binder materials as a function of temperature, Journal of Energetic Materials, Vol. 17, No. 1, 1999 pp. 1-47.

[32] J. F. Widener, M. W. Beckstead, Aluminum combustion modeling in solid propellant combustion products, AIAA Paper 98-3824, 1998. 
[33] R. Bunker, A. Prince, Hybrid rocket motor nozzle material predictions and results, Proceedings of the AIAA/SAE/ASME/ASEE 28th Joint Propulsion Conference and Exhibit, Paper 92-3591, July 1992.

[34] Laboratory data obtained with two inch diameter motors. Thiokol Corporation, 1989 (in [1], pp. 736).

[35] G. Lengelle, B. Fourest, J. C. Godon, C. Guin, Condensed phase behaviour and ablation rate of fuels for hybrid rocket propulsion, AIAA Paper 93-2413, 1993.

[36] D. A. Gordon, in "Solid Propellant Rocket Research," M. Summerfield, ed., Academic Press, New York, 1960, p. 271.

[37] R. D. Swami, A. Gany, Analysis and testing of similarity and scale effects in hybrid rocket motors, Acta Astronautica, Vol. 52, 2003, pp. 619-628.

[38] V. Stamatov, D. R. Honnery, and J. Soria, Visualization of flow development in hybrid rocket motors with high regression rates, Journal of Propulsion and Power, Vol. 21, No. 4, July-August 2005, pp. 613-618.

[39] CFX 5.7.1, "Basic Solver Capability Theory," CFX 5.7.1 Reference Guide, Ansys Inc., 2004.

[40] CFX 5.7.1, "Turbulence and Wall Function Theory," CFX 5.7.1 Reference Guide, Ansys Inc., 2004. 
[41] K. K. Kuo, "Principles of Combustion," Wiley, New York, 1986, Chapter 3, 7.

[42] CFX-TASCflow, "Primer Documentation," AEA Technology, Version 2.10, 2000, pp. 87-113.

[43] M. Tooley, ANSYS CFX Applications Specialist, private communication, August 8,2005 .

[44] D. C. Wilcox, "Turbulence Modeling for CFD," DCW Industries, California, 1998, pp.238-239.

[45] CFX 5.7.1, "Coupled Solver Theory," CFX 5.7.1 Reference Guide, Ansys Inc., 2004 .

[46] CFX 5.7.1, "Solution and Discretization Theory," CFX 5.7.1 Reference Guide, Ansys Inc., 2004.

[47] F. M. White, "Viscous Fluid Flow," McGraw-Hill, New York, 2nd Ed., 1991 pp. $415,435-436,483-484,487-489$.

[48] C. Yam and H. Dwyer, Investigation of the influence of blowing and combustion on turbulent wall boundary layers, Technical Note, AIAA Journal, Vol. 27, No. 3, March 1989, pp. 370-372.

[49] B. Holmes, ANSYS CFX Applications Specialist, private communication, May $17,2005$. 
[50] CFX 5.7.1, "Basic Capability Modelling," CFX 5.7.1 Reference Guide, Ansys Inc., 2004.

[51] B. J. McBride, S. Gordon, M. A. Reno, Coefficients for calculating thermodynamic and transport properties of individual species, NASA TM-4513, October 1993.

[52] R. Reid, J. Prausnitz, B. Poling, "The Properties of Gases and Liquids," 4th Ed., McGraw-Hill, New York, 1987, pp. 396, 516.

[53] C. K. Westbrook and F. L. Dryer, Simplified reaction mechanisms for the oxidization of hydrocarbon fuels in flames, Combustion Science and Technology, Vol. 27, 1981, pp. 31-43.

[54] G. C. Cheng, private communication, June 30, 2005.

[55] B. F. Magnussen and B. H. Hjertager, On mathematical modelling of turbulent combustion with special emphasis on soot formation and combustion, Sixteenth Symposium (International) on Combustion, MIT, Cambridge, Massachusetts, The Combustion Institute, August 15-20, 1976, pp. 719-728.

[56] I. S. Ertesvag and B. F. Magnussen, The eddy dissipation turbluence energy cascade model, Combustion Science and Technology, Vol. 159, No. 1-6, 2000, pp. $213-235$. 
[57] C. Y. Ma, T. Mahmud, P. H. Gaskell, E. Hampartsoumian, Numerical predictions of a turbulent diffusion flame in a cylindrical combustor using eddy dissipation and flamelet combustion models, Prodeedings of the Institution of Mechanical Engineers, Journal of Mechanical Engineering Science, Vol. 213, No. 7, 1999, pp. 698-705.

[58] CFX 5.7.1, "Radiation Theory," CFX 5.7.1 Reference Guide, Ansys Inc., 2004.

[59] J. P. Holman, "Heat Transfer," 7th Ed., McGraw-Hill Inc., New York, 1990, pp. $425-436$.

[60] M. J. Chiaverini, N. Serin, D. K. Johnson, Yeu-cheng Lu, K. K. Kuo, G. A. Risha, Thermal pyrolysis and combustion of HTPB-based solid fuels for hybrid rocket motor applications, AIAA Paper 96-2845, 1996.

[61] N. Kubota,"Survey of Rocket Propellants and Their Combustion", in Fundamentals of Solid-Propellant Combustion, edited by K. K. Kuo and M. Summerfield, Vol. 90, Progress in Aeronautics and Astronautics, AIAA, New York, 1984, pp. $38-40$.

[62] P. R. Bullen, D. J. Cheeseman, L. A. Hussain, A study of turbulent flow in pipe contractions, Proceedings of the Institution of Mechanical Engineers, Vol. 210, E3, 1996, pp. $171-180$. 
[63] D. L. Dean, Effects of fuel formulation on regression performance in hybrid motors, AIAA Paper 96-0886, January 1996.

[64] R. W. Humble, Fuel performance enhancements for hybrid rockets, AIAA Paper 2000-3437, July 2000. 